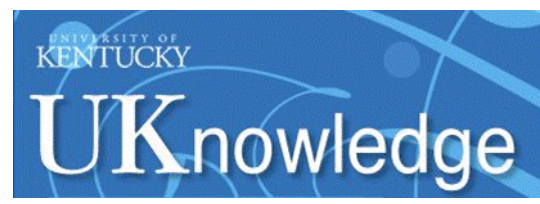

University of Kentucky

UKnowledge

\title{
APPROXIMATE ANALYSIS OF RE-ENTRANT LINES WITH BERNOULLI RELIABILITY MODELS
}

Chong Wang

University of Kentucky, cwanguky@gmail.com

Right click to open a feedback form in a new tab to let us know how this document benefits you.

\section{Recommended Citation}

Wang, Chong, "APPROXIMATE ANALYSIS OF RE-ENTRANT LINES WITH BERNOULLI RELIABILITY MODELS" (2007). University of Kentucky Master's Theses. 482.

https://uknowledge.uky.edu/gradschool_theses/482

This Thesis is brought to you for free and open access by the Graduate School at UKnowledge. It has been accepted for inclusion in University of Kentucky Master's Theses by an authorized administrator of UKnowledge. For more information, please contact UKnowledge@lsv.uky.edu. 


\title{
ABSTRACT OF THESIS
}

\section{APPROXIMATE ANALYSIS OF \\ RE-ENTRANT LINES WITH BERNOULLI RELIABILITY MODELS}

\begin{abstract}
Re-entrant lines are widely used in many manufacturing systems, such as semiconductor, electronics, etc. However, the performance analysis of re-entrant lines is largely unexplored due to its complexity. In this thesis, we present iterative procedures to approximate the production rate of re-entrant lines with Bernoulli reliability of machines. The convergence of the algorithms, uniqueness of the solution, and structural properties, have been proved analytically. The accuracy of the procedures is investigated numerically. It is shown that the approaches developed can either provide a lower bound or a closed estimate of the system production rate. Finally, a case study of automotive ignition component line with re-entrant washing operations is introduced to illustrate the applicability of the method. The results of this study suggest a possible route for modeling and analysis of re-entrant systems.
\end{abstract}

KEYWORDS: Re-entrant lines, Production rate, Bernoulli reliability, Last buffer first serve, Recursive procedure. 
By

Chong Wang

Dr. Jingshan $L i$

Director of Thesis

Dr. Yuming Zhang

Director of Graduate Studies

Nov.4, 2007 


\section{RULES FOR THE USE OF THESIS}

Unpublished Thesis submitted for the Doctor's degree and deposited in the University of Kentucky Library are as a rule open for inspection, but are to be used only with due regard to the rights of the authors. Bibliographical references may be noted, but quotations or summaries of parts may be published only with the permission of the author, and with the usual scholarly acknowledgments.

Extensive copying or publication of the thesis in whole or in part requires also the consent of the Dean of The Graduate School of the University of Kentucky.

A library that borrows this thesis for use by its patrons is expected to secure the signature of each user.

$\underline{\text { Name }}$

Date 


\section{THESIS}

Chong Wang

The Graduate School

University of Kentucky

2007 
APPROXIMATE ANALYSIS OF

RE-ENTRANT LINES WITH BERNOULLI RELIABILITY MODELS

\section{THESIS}

A thesis submitted in partial fulfillment of the requirements for the degree of Master of Science in the

College of Engineering at the University of Kentucky

$$
\text { By }
$$

Chong Wang

Lexington, Kentucky

Director: Dr. Jiangshan Li, Assistant Professor of Electrical Engineering

Lexington, Kentucky

2007

Copyright ${ }^{\oplus}$ Chong Wang 2007 


\section{DEDICATION}

Dedicated to my family. 


\section{ACKNOWLEDGMENTS}

I would like to thank my thesis advisor Dr. Jingshan Li for his guidance and support for my thesis. I would also like to thank my advisor Kozo Saito for his support and help and Dr. Yuming Zhang for his help.

I would also like to acknowledge my friends, my parents for their help and support. 


\section{TABLE OF CONTENTS}

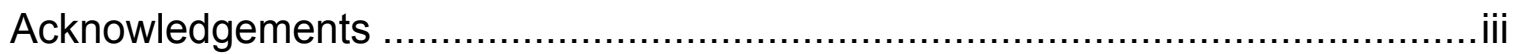

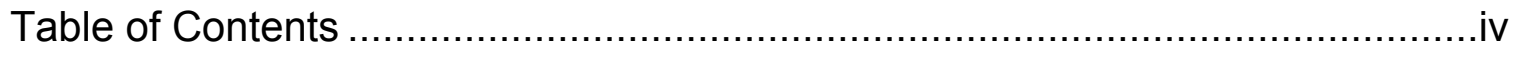

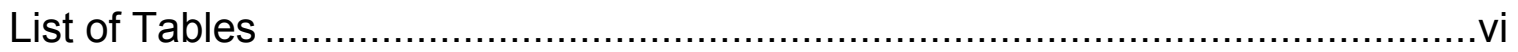

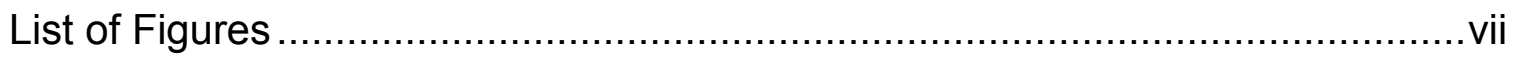

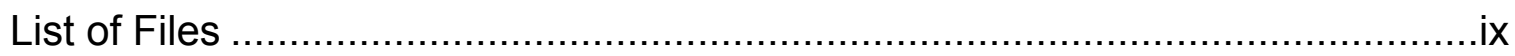

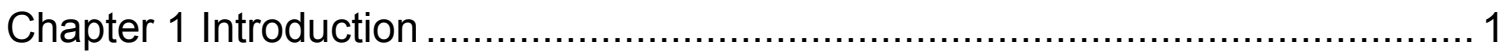

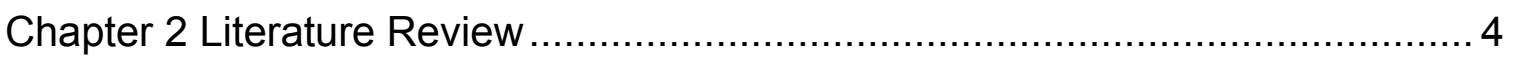

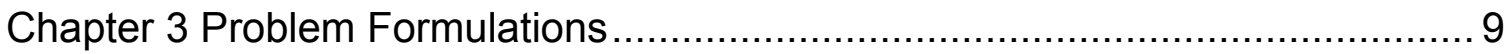

Chapter 4 Analysis of Re-entrant Lines ..................................................... 13

4.1 Re-entrant Line Model ......................................................... 13

4.2 Aggregation Method for Serial Production Lines ......................... 15

4.3 Recursive Procedure for Re-entrant Lines.................................. 21

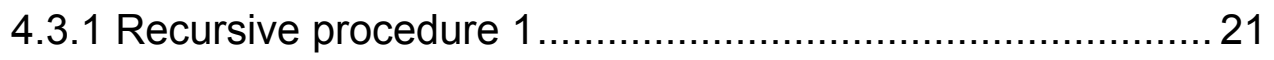

A) Analytical expression ................................................. 21

B) Convergence …..................................................... 21

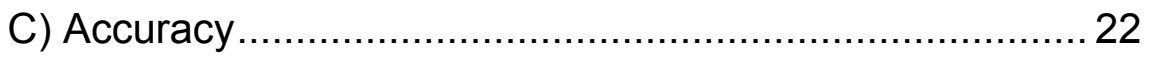

4.3.2 Modified recursive procedure ................................................. 25

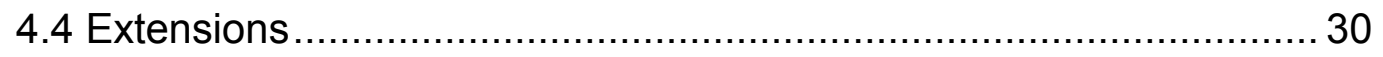

4.4.1 Re-entrant lines with different machine parameters for $1^{\text {st }}$ and $2^{\text {nd }}$ time jobs.................................................................. 30

A) Analytical expressions ............................................... 30

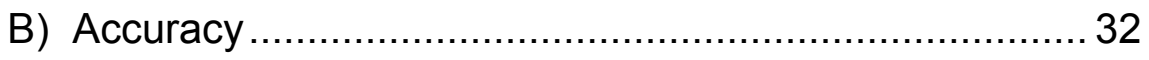

4.4.2 Re-entrant lines with more than two times of processing jobs 


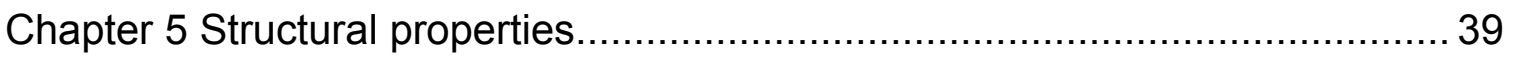

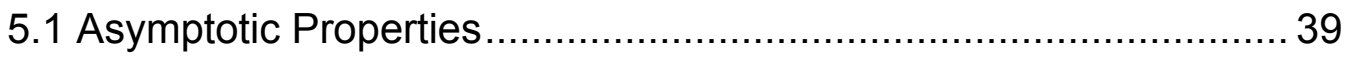

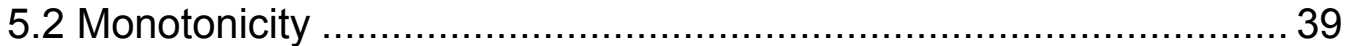

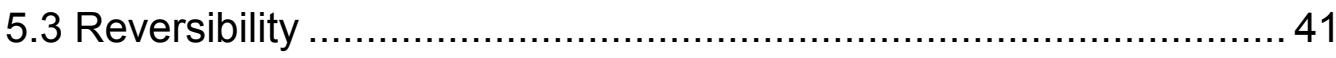

5.4 Policy Comparison between FBFS and LBFS ........................... 42

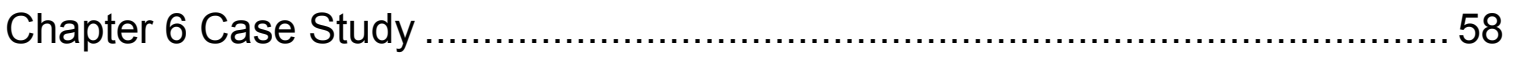

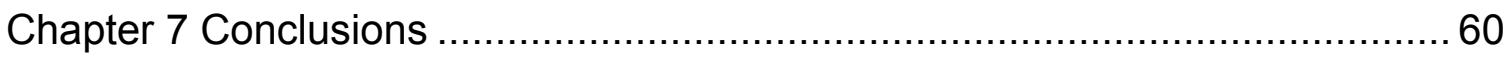

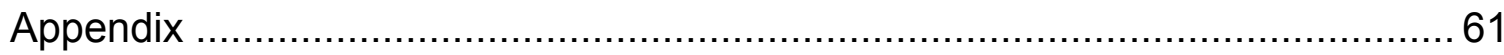

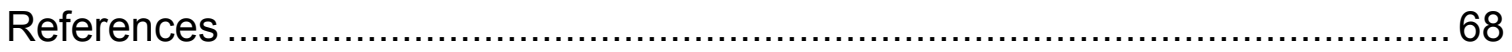

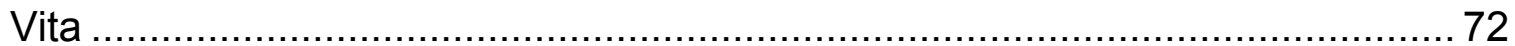




\section{LIST OF TABLES}

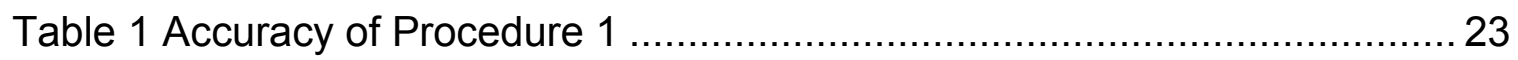

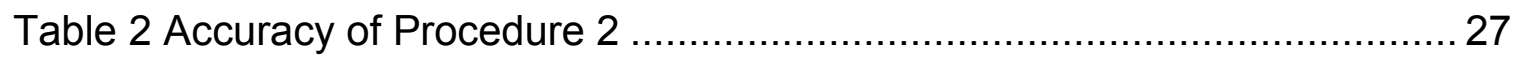

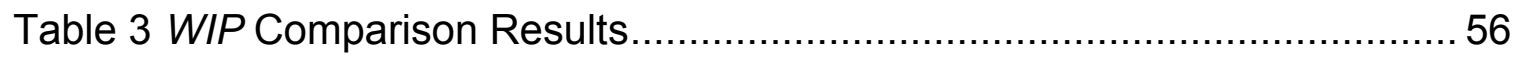

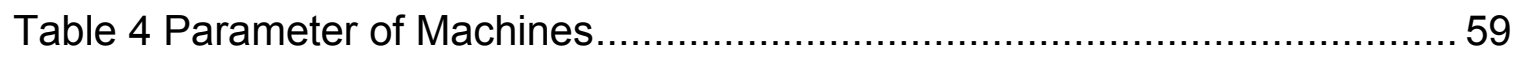

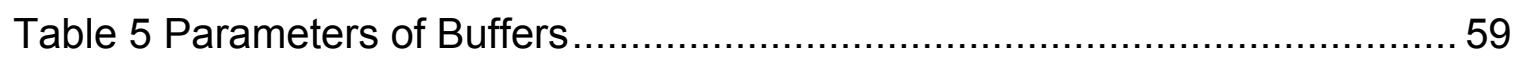




\section{LIST OF FIGURES}

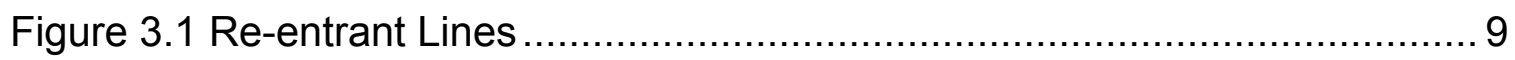

Figure 4.1 Two-machine Re-entrant Line .................................................. 13

Figure 4.2 Equivalent Four-machine Serial Line .......................................... 14

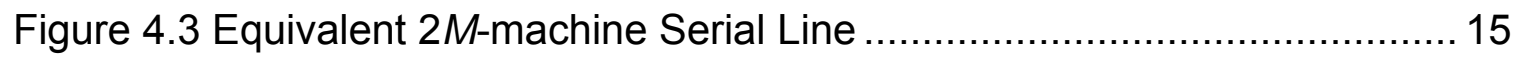

Figure 4.4 M-machine Bernoulli Production Line .......................................... 16

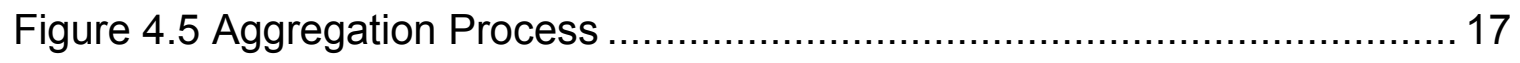

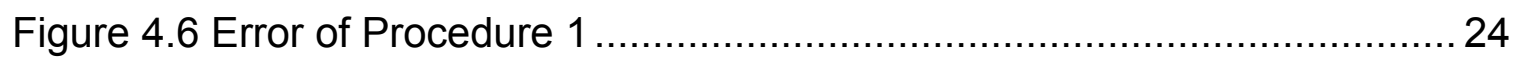

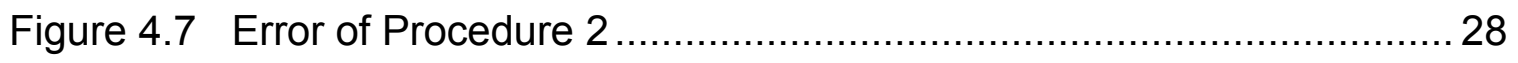

Figure 4.8 Re-entrant Line with Different Machine Parameters for 1st and 2nd

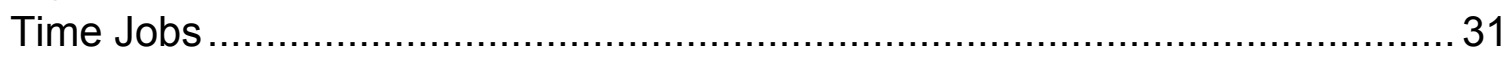

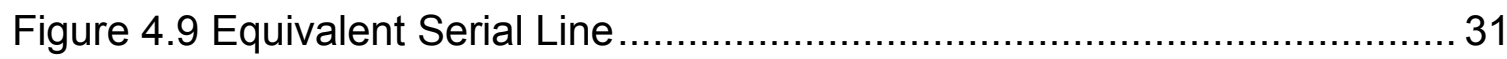

Figure 4.10 Error of Procedure 2 for Re-entrant Lines with Different Processing

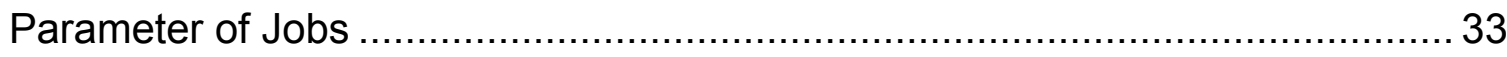

Figure 4.11 Re-entrant Line with Three-time-processing of Jobs .................... 37

Figure 4.12 Equivalent Serial Line of Line in Figure 4.11 ............................. 38

Figure 4.13 Errors for 4-machine, 3-Layer Re-entrant Line using Procedures 1 and 2

Figure 5.1 PR as Function of Buffer Capacity …........................................... 40

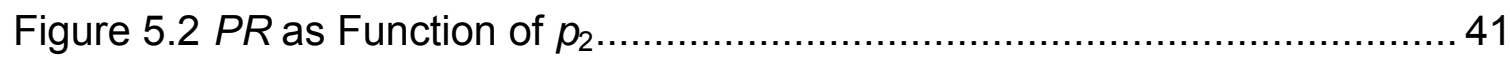

Figure 5.3 Reversed Re-entrant Lines...................................................... 42

Figure 5.4 Two-machine Re-entrant Line with FBFS Policy ............................. 43

Figure 5.5 Equivalent Serial Line of the Re-entrant Line in Figure 5.4 ............. 44

Figure 5.6 PR Comparison using FBFS and LBFS ..................................... 46

Figure 5.7 Lead Time Comparison using FBFS and LBFS ............................. 50 
Figure 5.8 WIP Comparison using FBFS and LBFS.

Figure 6.1 Structure of Ignition Component Processing System ……...............58

Figure 6.2 Equivalent Serial Line for Re-entrant Line in Figure 6.1 ..................59 
LIST OF FILES

File 1 Thesis.pdf $882 \mathrm{~KB}$ 


\section{CHAPTER 1}

\section{INTRODUCTION}

Performance analysis is important for design, operation and management of production systems. Substantial amount of research attention has been paid during the last fifty years. For two-machine lines, exact analytical results exist, while for longer lines and assembly systems, aggregation and decomposition methods have been developed to approximate system performance. Such methods have been extended to more complex systems, for instance, systems with rework loops, parallel lines, split, merge and closed loop systems, etc. (see reviews [1]-[3] and monographs [4]-[8]).

In addition to above systems, re-entrant lines have been widely encountered in many manufacturing systems, such as semiconductor, electronics, automotive, etc. ([9]-[13]). In such lines, the parts visit some machines multiple times. For example, in semiconductor manufacturing, the production process typically is carried out layer by layer by imprinting multiple layers of chemical patterns on the wafer ([14]). Similar situation occurs in automotive industry as well. In powertrain manufacturing plants, some ignition components need to be processed multiple times. For example, for fuel injectors, the armatures, needles or seats typically reenter the central washers multiple times to keep clean. The re-entrant characteristics also exist in the future fuel cell and nano-manufacturing systems. Therefore, the analysis, design and operation management of re-entrant lines are of significant importance. However, the performance analysis of re-entrant lines is limited due to its complexity. Much of the available work on re-entrant lines 
focuses on investigating the scheduling and control policies. Queueing network models, Petri net approaches, and discrete event simulations are the main tools used for performance evaluation in such studies (see, for instance, representative papers [10]-[19]). Most of them assume either infinite buffer capacities or reliable processing of materials.

In spite of these efforts, there is still a need to develop an accurate analytical tool to estimate the performance of re-entrant lines, in particular, lines with unreliable machines and finite buffers. Such a tool would be desirable and useful for many large volume manufacturing industries. The goal of this thesis is to contribute to this end.

Specifically, we develop an analytical method to estimate the production rate of a re-entrant line. The basic idea of the method is to equivalent the $M$ machine re-entrant line into a $2 M$-machine serial line. The first $M$ machines are dedicated for first time jobs and the latter $M$ machines for second time jobs. The machine parameters are modified to take into account the multiple processing of jobs. Two iterative procedures have been developed to obtain these parameters recursively. It is proved that these procedures are convergent and the unique steady state solution exists. The main contribution of this thesis is the development of such procedures which can be used to approximate the production rates of re-entrant lines.

The remaining of the thesis is structured as follows: Chapter 2 reviews the literature and Chapter 3 formulates the problem. The modeling and analysis method is presented in Chapter 4. Chapter 5 studies the structural properties of 
re-entrant lines. Chapter 6 introduces a case study in designing ignition component line with re-entrant washing operations. Finally, Chapter 7 presents the conclusion of the thesis. All proofs are provided in the Appendix. 


\section{CHAPTER 2}

\section{LITERATURE REVIEW}

Due to the widely application of re-entrant lines in semiconductor manufacturing systems, the need to understand and control the re-entrant lines has motivated great amount of research in this area ([14]). In this chapter, literatures about different methods are reviewed.

Most of the studies addressing the control and scheduling policies in re-entrant lines (see representative paper [14]). Priority scheduling policy is typical in re-entrant lines ([14]). First Buffer First Serve (FBFS) policy, Last Buffer First Serve (LBFS) policy, Earliest Due Day (EDD) policy, and Least Slack (LS) policy, are the typical policies studied and implemented in semi-conductor manufacturing systems. In LBFS policy, more processed jobs have higher priority than less processed ones. Such policy is also used in many other manufacturing systems, for instance, production systems with rework loops ([20]). In addition, the stabilities and performances of different policies are also discussed ([23]). It is proved that FBFS, LBFS, EDD and LS are all stable. These results are typically verified by simulations. The simulation results show that LBFS and LS policies have advantages at different work loads. LFBS may be the best policy for minimizing mean delay at high load factors, and LS may be the best policy for minimizing variance of the delay. 
The re-entrant lines have been studied using various methods, including queuing models, discrete event simulation, fluid model and Petri networks, etc. ([9]-[24]).

Queueing theory has been extensively studied to model computer systems, communication and manufacturing systems ([22, 24]). Multi class queue models have been employed to study re-entrant manufacturing lines. A general multi class queue is defined as follows: There are multiple stations in the network, with the entire customer following the same route of processing through different stations at different stages. The customer at stage $k$ is designated as class $k$ customers. In manufacturing environment, one can consider the customers to be the parts that are going to be processed by different machines at different stages, and then the multi class queueing model is similar to a re-entrant line. Thus, it can be applied to study the properties of re-entrant lines. Bramson [24] studies the queue limit at high traffic load, and proves the heavy traffic limit theorem for re-entrant lines with FBFS and LBFS policies.

Fluid model (also known as the functional strong law-of-large-numbers) ([25]) is also employed to study multi class queueing network as in re-entrant production lines. Dai $([25,26])$ studies the fluid approximation and the stability for a multiclass queueing network. It is proved that a scheduling policy is stable if the corresponding fluid model is stable ([25]). Stability and instability of fluid model are studied in [26], where stability of First Buffer First Serve (FBFS) and Last Buffer First Served (LBFS) policies for re-entrant lines are addressed. 
Due to the complexity of semiconductor manufacturing system, applying queueing theory into semiconductor manufacturing systems modeling faces changelings. Modeling of re-entrant production line is one example. According to the literature, most of the analysis is cumbersome and is mostly limited to the study of different scheduling policies, such as FBFS and LBFS. The stability of such policies is well studied using queueing models and analogue fluid model. However, typically, only a performance bound can be obtained using queueing and fluid models, the production rate of the system has not been analyzed accurately, which limits its application to production line design. In this work, we intend to develop novel method.

In addition to queueing and fluid models, Petri net approach provides another way of modeling re-entrant lines. Choi and Reveliotis ([19]) present an analytical framework for the modeling, analysis and control of flexibly automated re-entrant lines, using Generalized Stochastic Petri Nets (GSPN's). They propose a study on time-based aspects of the system behavior, analytical formulation for the re-entrant line scheduling problem, and a qualitative characterization of the optimal scheduling policy. However, a limitation of this method is that it requires the enumeration of the state space, which explodes very fast as production line becomes more complex. This is also one of the limitations to apply Petri net approach in modeling re-entrant lines.

Since the queueing models and Petri net approach are limited to provide accurate analysis, simulations are widely applied in cycle time estimation and performance analysis of semiconductor manufacturing systems ([22]). 
Building the system model and using high quantity of iterations can give relatively accurate result to guide production line planning and scheduling, also validation of analytical models.

Wein ([28]) studies the impact of scheduling on the performance of semi-conductor wafer fabrication line using a simulation model of a fictitious semiconductor wafer production line. A variety of input control and sequencing rules are evaluated based on mean throughput time (cycle time). Simulation results indicate that scheduling has a significant impact on average throughput time.

In paper [27], a simulation based optimization approach is employed to study capacity allocation rules in re-entrant manufacturing lines. Several rules for production and capacity allocation are analyzed. Infinitesimal perturbation analysis (IPA) method is studied and IPA derivative expressions are formulated and validated. These derivatives can be applied to study the optimal configuration of the re-entrant lines. However, computational intensive effort is required for this method.

Although simulation can provide significant help for analysis of re-entrant lines, the limitation of simulation models is also apparent. First, it is a case by case modeling method. Small changes in production model can incur dramatic change in simulation, especially computational part. Second, it requires large quantity of input data, about equipment details work-in-process (WIP) management policies, and details about the products ([22]). In addition, it is time consuming and the costs for different 
simulations are relatively high, even with higher speed computer systems. Moreover, it cannot give insight of the production systems and cannot answer those 'what if' questions ([8]), especially in the design stage. Therefore, the study of analytical model for re-entrant line in a more time efficient manner is of great importance. 


\section{CHAPTER 3}

\section{PROBLEM FORMULATIONS}

A typical structure of a re-entrant line is shown in Figure 3.1, where the circles represent machines and the rectangles are buffers. The dash lines in the circles depict the product flow in the system. The following assumptions address the machines, the buffers, and their interactions.

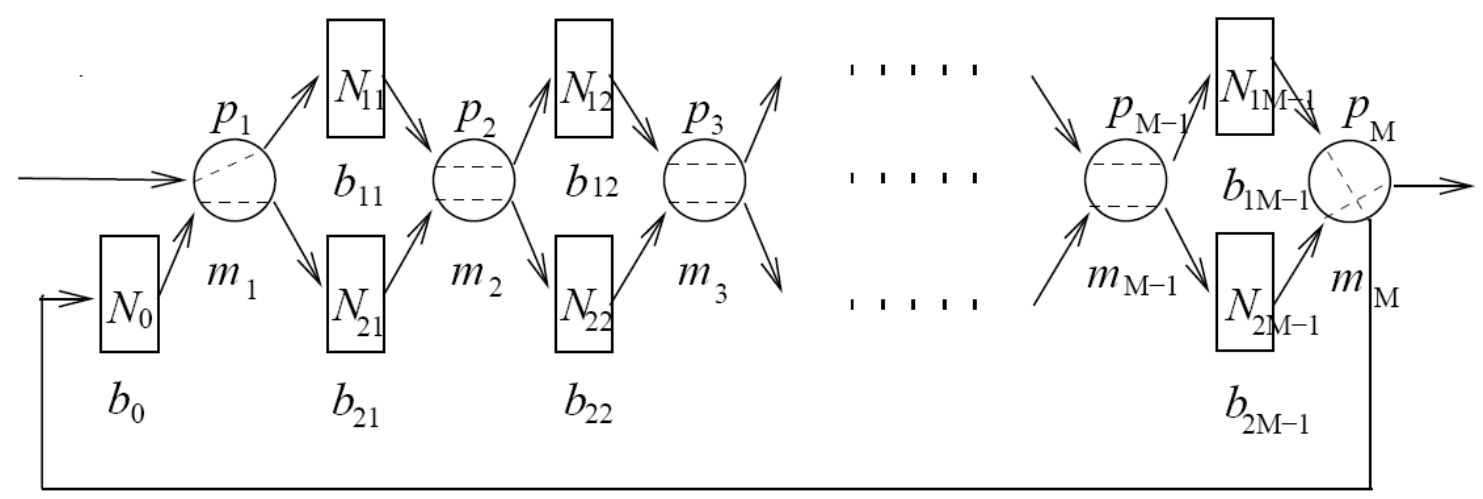

Figure 3.1 Re-entrant Lines

1) The system consists of $M$ machines and $2 M-1$ buffers. The first time jobs are processed at machines $m_{i}, i=1, \ldots, M$, and buffers $b_{1 i}, i=1, \ldots, M-1$, between two consecutive machines. After first time processing at machine $m_{M}$, all jobs are sent to buffer $b_{0}$, waiting for second time processing. Then the jobs are reprocessed at machines $m_{i}, i=1, \ldots, M$, but through buffers $b_{2 i}, i=1, \ldots, M-1$. Jobs leave the system after being processed at $m_{M}$ for the second time.

2) All machines have identical processing times. The time is slotted as cycle time. 
3) Each machine $m_{i}, i=1, \ldots, M$, is characterized by its reliability $p_{i}$, i.e., at each cycle, $m_{i}$ has probability $p_{i}$ to be up and $1-p_{i}$ to be down. When it is up, it is capable of processing a part. When the machine is down, no production takes place.

Remark 1: Assumptions 2) and 3) formulate the Bernoulli reliability model of the machines. In our experience, many production systems, such as assembly type systems, where the machine downtime is comparable to machine cycle time, obey this reliability model. In such systems, the majority of the machine breakdowns are due to pallet jam, push button stop, etc., and only a short period of time is needed to correct these problems. In contrast, exponential machine reliability models are typically suitable for operations where relative long repair times, compared to their cycle times, are required to fix the machine breakdowns. For lines with longer downtimes, an exponential-Bernoulli (E-B) transformation has been introduced in [8], where exponential lines can be transformed into Bernoulli lines with acceptable accuracy. In this thesis, we focus our work on Bernoulli re-entrant lines. Lines with exponential reliability machines can be studied in future work.

4) Each buffer $b_{k}, k=11,12, \ldots,(1, M-1), 21,22, \ldots,(2, M-1)$, and 0 , has capacity $N_{k}, \quad 0<N_{k}<\infty$.

5) Machine $m_{i}, i=1, \ldots, M-1$, is blocked by the first (respectively, second) time job if buffer $b_{1 i}$ (respectively, $b_{2 i}$ ) is full and machine $m_{i+1}$ does not take a part from it. Machine $m_{M}$ is blocked by the first time job if buffer $b_{0}$ is full and machine 
$m_{1}$ does not take a part from $b_{0}$. Machine $m_{M}$ is never blocked by the second time job.

6) The second time jobs have higher priorities than the first time ones. In other words, machine $m_{i}, i=2, \ldots, M-1$, always takes a part from buffer $b_{2, i-1}$ if it is not empty and $m_{i}$ is not blocked by $b_{2 i}$, otherwise it will take a part from buffer $b_{1, i-1}$ if it is not empty and $m_{i}$ is not blocked by $b_{1 i}$. Machine $m_{1}$ takes parts from buffer $b_{0}$ if it is not empty and $m_{1}$ is not blocked by $b_{11}$, otherwise a new part will be loaded to be processed at machine $m_{1}$. Machine $m_{M}$ will take part from $b_{2 M-1}$ if it is not empty, otherwise $m_{M}$ loads from $b_{1, M-1}$ if it is not empty and $m_{M}$ is not blocked by $b_{0}$.

Remark 2: It has been shown in the literature (for instance [14]) that Last Buffer First Serve (LBFS) is the best proven policy for reducing mean delay. Therefore, we analyze the re-entrant line with LBFS policy first in this work. The First Buffer First Serve (FBFS), i.e., buffers $b_{1 i}, i=1, \ldots, M-1$ have higher priorities is also investigated. A comparison between LBFS and FBFS policies is carried out and presented in Chapter 5.

7) Machine $m_{i}, i=2, \ldots, M$, is starved if buffers $b_{1, i-1}$ and $b_{2, i-1}$ are empty. Machine $m_{1}$ is never starved by the first time job.

Assumptions 1)-7) define the system under consideration. In the time scale of the time slot, these define a stationary, ergodic Markov chain. The steady state of the chain is considered in this work. We refer to this steady state as the normal system operations. Let $P R$ be the production rate of the system, 
i.e., the average number of parts produced by the last machine per time slot. The problem addressed in this work is formulated as follows:

Given production system 1)-7) develop a method for evaluating the production rate as a function of the system parameters and study the system theoretic properties.

The solutions to the above problem are presented in Chapters 4 and 5 in this thesis. 


\section{CHAPTER 4}

\section{ANALYSIS OF RE-ENTRANT LINES}

\subsection{Re-entrant Line Model}

The main difficulty of analyzing re-entrant line is that the machines are used for multiple processing of jobs. In addition to the complexity typically existed in serial lines, more difficulties coming from the allocation of machine capacity to multiple processing of jobs, the priority loading and the dedicated dispatching policies, etc., make the exact analysis of system performance all but impossible. Therefore, approximation method is pursued in this work.

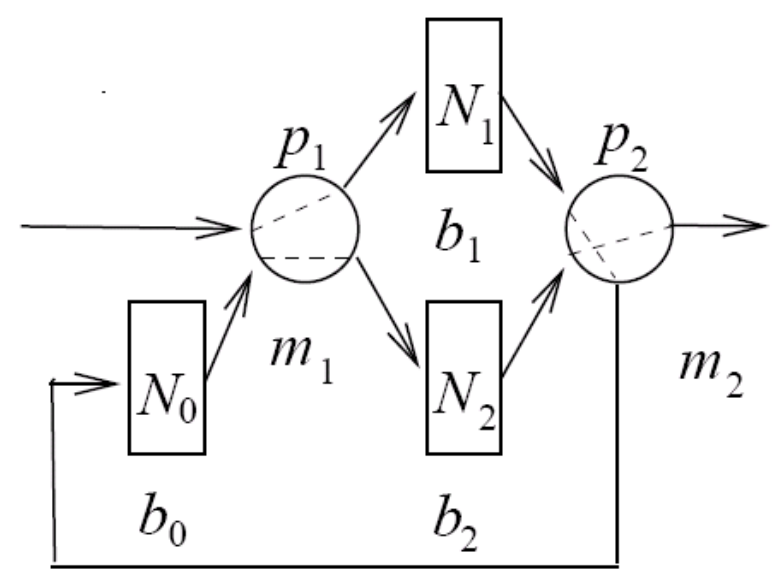

Figure 4.1 Two-machine Re-entrant Line

The idea of the approximation is illustrated as follows: Consider a twomachine re-entrant line depicted in Figure 4.1. Denote the production rates of machine $m_{i}, i=1,2$, to the $j$-th time jobs, $j=1,2$, as $p r^{(j)}{ }_{i}$. It is clear that $m_{2}$ works on second time jobs as long as buffer $b_{2}$ is not empty. Therefore, the availability of $m_{2}$ to second time jobs is $p_{2}$, which implies that the production rate on second 
time jobs, $p r_{2}^{(2)}$, equals to the probability that $m_{2}$ is up and not starved by $b_{2}$. Machine $m_{2}$ is available to first time jobs only when $m_{2}$ is up but could not process second time jobs (i.e., $b_{2}$ is empty). It is equivalent that a machine with reliability $p_{2}-p r^{(2)}{ }_{2}$ is available to first time jobs. Therefore, the production rate of $m_{2}$ to first time jobs, $p r^{(1)}{ }_{2}$, can be approximated by: $p_{2}-p r^{(2)}{ }_{2}$ subtracts the probabilities of blockage and starvation by buffers $b_{0}$ and $b_{1}$, respectively.

Similarly, machine $m_{1}$ has higher priority to second time jobs. Thus, its availability is $p_{1}$, and $m_{1}$ is working on second time jobs if it is not blocked by $b_{2}$ or starved by $b_{0}$, and we denote this production rate as $p r^{(2)}$. Machine $m_{1}$ is working on first time jobs only when second time processing is not possible (blocked by $b_{2}$ or starved by $b_{0}$ ). We can approximate this machine as $p_{1}-p r^{(2)}$. Therefore, two-machine re-entrant line can be equivalent into a four-machine serial line, where the first two pseudo machines, $m_{1}^{\prime}$ and $m_{2}^{\prime}$, represent the first time processing, and the last two machines, $m_{1}$ and $m_{2}$, characterize the second time processing (see Figure 4.2).

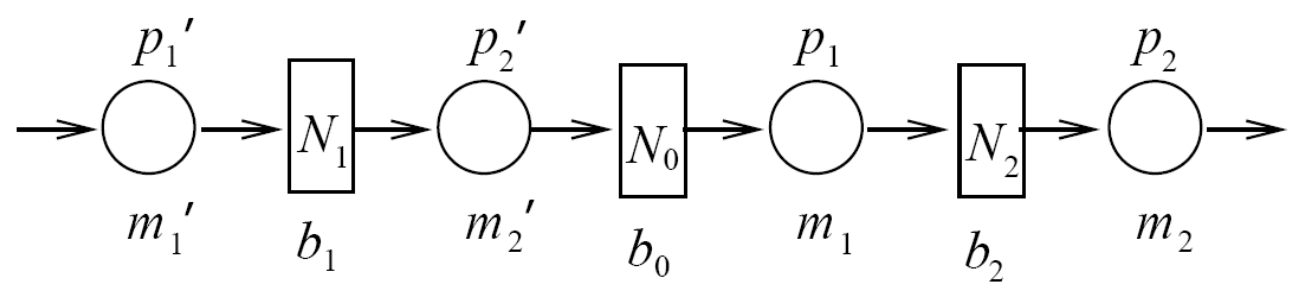

Figure 4.2 Equivalent Four-machine Serial Line

Due to conservation of flow, the system production rate will be equal to the production rate for all machines and for both the first and second time jobs, i.e., 
$p r^{(1)}{ }_{1}=p r^{(2)}{ }_{1}=p r^{(1)}{ }_{2}=p r^{(2)}{ }_{2}=p r$. Therefore, the parameters of machines $m_{i}$ and $m_{i}^{\prime}$, $i=1,2$, equal to $p_{i}$ and $p_{i}-p r$, respectively. Analogously, we can extend this idea to the general $M>2$-machine re-entrant line (Figure 3.1) by a $2 M$-machine serial line, as shown in Figure 4.3, where the first $M$ machines are pseudo machines with parameters $p_{i}-p r$, and next $M$ machines have reliability $p_{i}, i=1, \ldots, M$.

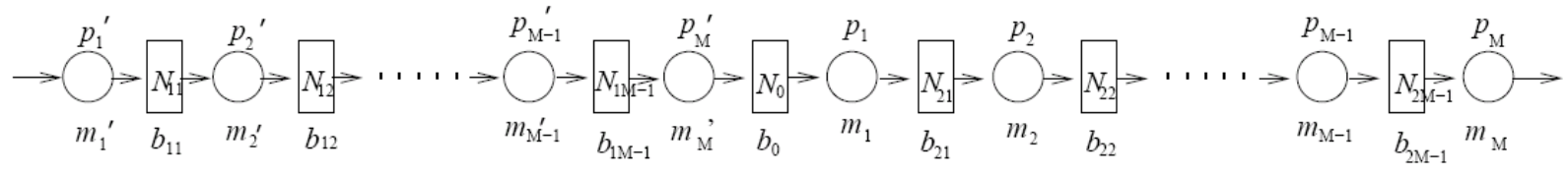

Figure 4.3 Equivalent 2M-machine Serial Line

Based on these equivalent serial lines, re-entrant lines can be analyzed and approximated using approaches developed for serial lines. For Bernoulli machine reliability models, aggregation method has been introduced to study the equivalent serial lines ([8]). To make this thesis self-contained, we provide the aggregation method for Bernoulli serial lines next ([8]).

\subsection{Aggregation Method for Serial Production Lines}

Consider a serial production line illustrated in Figure 4.4, closed form analytical solution only exists for two-machine lines. For lines with more than two machines, due to its complexity (mainly because of the interacting among all machines and buffers in the line), iterative aggregation method is introduced. The idea of the aggregation is as follows: 
First, we aggregate the last two machines, $m_{M-1}$, and $m_{M}$ into a single Bernoulli machine denoted as $m^{b}{ }_{M-1}$, where $b$ stands for backward aggregation. The aggregated machine has the same production rate of the two-machine line. Figure 4.5(a) depicts the backward aggregation process. The Bernoulli probability parameter, $p^{b}{ }_{M-1}$, of this machine can be calculated ([8]).

Next, we aggregate this machine, i.e., $m_{M-1}{ }^{b}$, with the upstream machine $m_{M-2}$ and obtain another aggregated machine $m_{M-2}{ }^{b}$. Continue this process till the first machine in the line.

Then all the machines in this line are aggregated into machine $m_{1}{ }^{b}$.

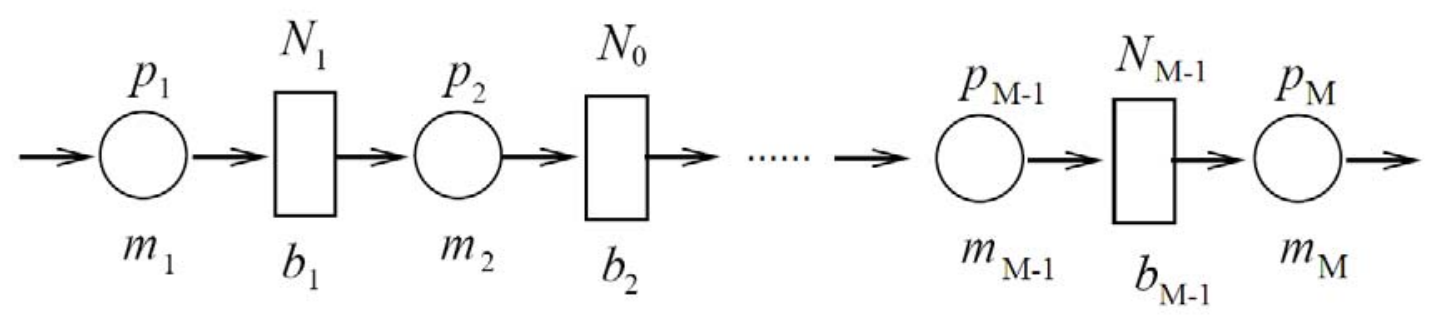

Figure 4.4 M-machine Bernoulli Production Line 


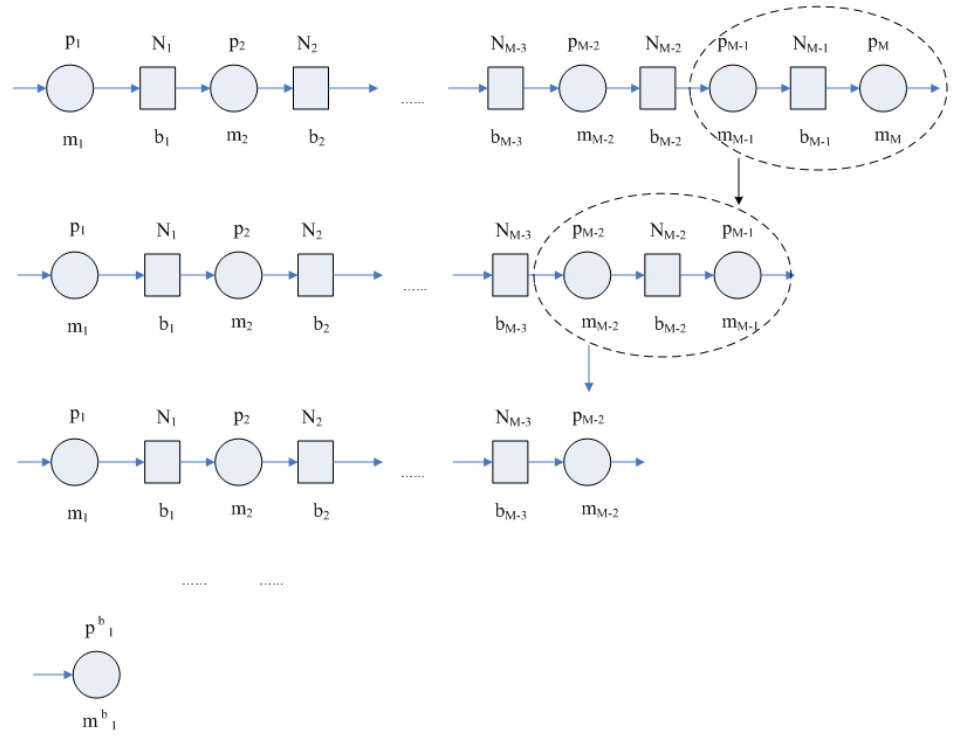

(a) Backward Aggregation

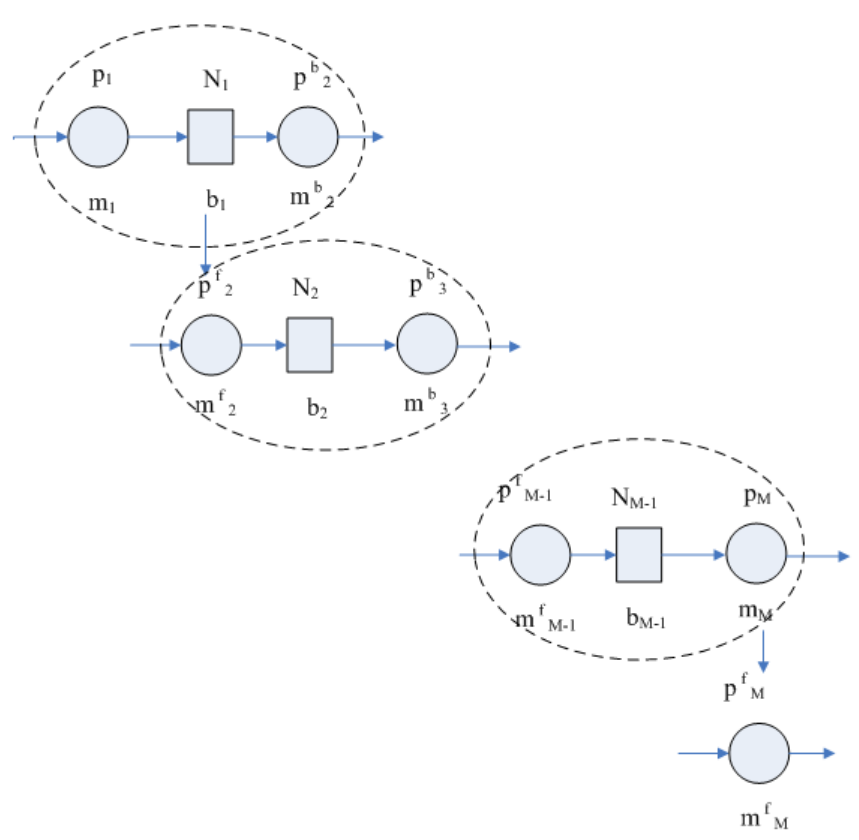

(b) Forward Aggregation

Figure 4.5 Aggregation Process 
Since the backward aggregation does not consider the impact of starvation, the forward aggregation is introduced next. First we aggregate the first machine $m_{1}$ with the aggregated machine $m_{2}{ }^{b}$, to obtain a new aggregated machine, denoted as $m_{2}{ }^{f}$, in which $f$ denotes forward aggregation. The equivalent Bernoulli parameter $p_{2}{ }^{f}$ is calculated. Next, we aggregate $m_{2}{ }^{f}$ with $m_{3}{ }^{b}$ to get $m_{3}{ }^{f}$. This process is then carried on until all the machines are aggregated into $m_{M}{ }^{f}$ (see Figure 4.5(b)). This finishes the first iteration of the aggregation.

Next, iterations are employed to improve the accuracy of the aggregation. In the second iteration, $m_{M}$ is aggregated with $m_{M-1}^{f}$ to obtain $m_{M-1}^{b}$, and $m^{b}{ }_{M-1}$ is aggregated with $m_{M-2}^{f}$ into $m_{M-2}^{b}$. This process is continued till the backward procedure is finished. Then forward aggregation is carried out again. The process is iterated back and forth until it is convergent.

The recursive procedure described above can be expressed using the following mathematical equations ([8]):

$$
\begin{gathered}
p_{i}^{b}(s+1)=p_{i}\left[1-Q\left(p_{i+1}^{b}(s+1)\right), p_{i}^{f}(s), N_{i}\right] \\
i=1, \ldots, M-1, \\
p_{i}^{f}(s+1)=p_{i}\left[1-Q\left(p_{i+1}^{f}(s+1)\right), p_{i}^{b}(s), N_{i}\right] \\
i=2, \ldots, M-1, \\
s=0,1,2, \ldots, \\
p_{i}^{f}(0)=p_{i}, i=1, \ldots, M
\end{gathered}
$$

with initial conditions

and boundary conditions 


$$
\begin{array}{ll}
p_{1}^{f}(s)=p_{1}, & s=0,1,2, \ldots, \\
p_{M}^{b}(s)=p_{M}, & s=0,1,2, \ldots,
\end{array}
$$

and

$$
\begin{gathered}
Q(x, y, N)= \begin{cases}\frac{(1-x)(1-a)}{1-\frac{x}{y} \alpha^{N}}, & \text { if } x \neq y, \\
\frac{(1-x)}{N+1-x}, & \text { if } x=y,\end{cases} \\
\alpha=\frac{x(1-y)}{(1-x) y}
\end{gathered}
$$

It is proved that (for details, see [8]) that sequences, $p_{i}^{f}(s)$,

$i=1,2, \ldots, M$, and $p_{i}^{b}(s), i=1,2, \ldots, M-1$, are convergent. Then the following limits exist:

$$
\begin{aligned}
& p_{i}^{f}:=\lim _{s \rightarrow \infty} p_{i}^{f}(s), \quad i=1, \ldots, M, \\
& p_{i}^{b}:=\lim _{s \rightarrow \infty} p_{i}^{b}(s), \quad i=1, \ldots, M .
\end{aligned}
$$

When the procedure converges, the estimation of production rate is obtained:

$$
\begin{aligned}
& \widehat{P R}=p_{M}^{f}=p_{1}^{b} \\
& =p_{i+1}^{b}\left[1-Q\left(p_{i}^{f}, p_{i+1}^{f}, N_{i}\right)\right] \\
& =p_{i}^{f}\left[1-Q\left(p_{i+1}^{f}, p_{i}^{f}, N_{i}\right)\right], \\
& i=2, \ldots, M-1 .
\end{aligned}
$$


In addition, the work-in-process (WIP), i.e., the steady state occupancy of buffer $i$ can be calculated:

$$
\widehat{W I P_{i}}= \begin{cases}\frac{p_{i}^{f}}{p_{i+1}^{b}-p_{i}^{f} \alpha^{N_{i}}\left(p_{i}^{f}, p_{i+1}^{b}\right)}\left(\frac{1-\alpha^{N_{i}}\left(p_{i}^{f}, p_{i+1}^{b}\right)}{1-\alpha\left(p_{i}^{f}, p_{i+1}^{b}\right)}-N_{i} \alpha^{N_{i}}\left(p_{i}^{f}, p_{i+1}^{b}\right)\right), & \text { if } p_{i}^{f} \neq p_{i+1}^{b}, \\ \frac{N_{i}\left(N_{i}+1\right)}{2\left(N_{i}+1-p_{i}^{f}\right)} & \text { if } p_{i}^{f}=p_{i+1}^{b}, \\ i=1, \ldots, M-1 .\end{cases}
$$

The estimation of total WIP is

$$
\widehat{W I P}=\sum_{i=1}^{M-1} \widehat{W I P}
$$

It is shown in [8] that monotonicity and reversibility hold in serial lines. The production rate of a serial production line is monotonically increasing with respect to machine reliability and buffer capacity. The production rate of a revised serial line is identical to that of the original line, in other words,

$$
\begin{gathered}
P R\left(p_{1}, p_{2}, \ldots, p_{M}, p_{1}, \ldots, p_{M}, N_{1}, N_{2}, \ldots, N_{M-1}\right) \\
\quad=P R\left(p_{M}, p_{M-1}, \ldots, p_{1}, N_{M-1}, N_{M-2}, \ldots, N_{1}\right) .
\end{gathered}
$$

Moreover, the line is asymptotically stable, when all $N_{i}, i=1, \ldots, M-1$, are approaching infinity,

$$
\lim _{N_{i} \rightarrow \infty, \forall i} P R=\min \left\{p_{1}, p_{2}, \ldots, p_{M}\right\} .
$$




\subsection{Recursive Procedure for Re-entrant Lines}

\subsubsection{Recursive procedure 1}

\section{A) Analytical expression}

Introduce operator $P R\left(p_{1}, \ldots, p_{M}, N_{1}, \ldots, N_{M-1}\right)$ to denote the procedure for production rate calculation of a M-machine serial line introduced above. Using this operator, the following recursive procedure for re-entrant line 1)-7) is developed.

Procedure 1:

$$
\begin{gathered}
p_{i}^{\prime}(s+1)=p_{i}-\operatorname{pr}(s), i=1, \ldots, M, \\
\operatorname{pr}(s+1)=\operatorname{PR}\left(p_{1}^{\prime}(s+1), \ldots, p_{M}^{\prime}(s+1), p_{1}, \ldots, p_{M}, N_{11}, \ldots, N_{1, M-1}, N_{0}, N_{21}, \ldots, N_{2, M-1}\right), \\
s=0,1,2, \ldots,
\end{gathered}
$$

where

$$
\operatorname{pr}(0)=P R\left(p_{1}, \ldots, p_{M}, p_{1}, \ldots, p_{M}, N_{11}, \ldots, N_{1, M-1}, N_{0}, N_{21}, \ldots, N_{2, M-1}\right) .
$$

\section{B) Convergence}

Let $\widehat{P R}_{1}$ denote the production rate obtained, if Procedure 1 is convergent, where subscript "1" indicates the first procedure. It is shown below that this procedure does lead to a convergent result.

Theorem 4.1: Under assumptions 1)-7), Procedure 1 is convergent, therefore, the following limit exists:

$$
\lim _{s \rightarrow \infty} \operatorname{pr}(s):=\widehat{P R}_{1}
$$

Proof: See Appendix. 
Corollary 4.1 Under assumptions 1)-7), the steady state equations of Procedure 1 has a unique solution.

Proof: See Appendix.

Thus, an estimate of the production rate of the re-entrant line in steady state, $\widehat{P R}_{1}$, is obtained.

\section{C) Accuracy}

The accuracy of the approximation is investigated numerically. Specifically, we consider $M$-machine re-entrant lines, where $M \in\{2,3,5,8,10,11,15,20\}$. For each $M$, we construct 20 lines by randomly and equiprobably selecting machine and buffer parameters from the following sets:

$$
\begin{aligned}
& p_{i} \in[0.75,0.95], \\
& N_{i} \in\{1,2,3,4,5\} .
\end{aligned}
$$

As a result, a total of 160 re-entrant lines are investigated. For each line, both analytical method using Procedure 1 and simulation approach are pursued to evaluate system production rate. In each simulation, 10,000 cycles of warm-up time are assumed, and the next 100,000 cycles are used for collecting steady state statistics. 20 replications are carried out to obtain the average production rate, with $95 \%$ confidence intervals consistently ranging within \pm 0.0002 . Such simulation settings are used throughout the numerical experiments carried out in this thesis. The differences between analytical and simulation results are 
evaluated as:

$$
\varepsilon_{1}=\frac{\widehat{P R_{1}}-P R}{P R} \cdot 100 \%,
$$

where $P R$ and $\widehat{P R}_{1}$ are the production rates obtained by simulation and recursive procedure, respectively.

The results of this investigation are illustrated in Figure 4.6. It is shown that in all the cases we studied, Procedure 1 provides a lower bound for production rate estimation. Table 1 presents the tightness of such a bound. It is observed that the bound is tighter for shorter lines, and the average discrepancy is typically within $10 \%$. Since the bound is relatively tight, and it is conservative, the procedure can be a useful tool for design and analysis of re-entrant lines.

Table 1 Accuracy of Procedure 1

\begin{tabular}{|c|c|c|c|c|c|c|c|c|}
\hline $\begin{array}{c}\text { No. of } \\
\text { Machines }\end{array}$ & 2 & 3 & 5 & 8 & 10 & 11 & 15 & 20 \\
\hline$\overline{\left|\varepsilon_{1}\right|}(\%)$ & 5.92 & 7.6 & 9.26 & 9.90 & 9.60 & 10.08 & 9.06 & 9.50 \\
\hline $\max \overline{\left|\varepsilon_{1}\right|}(\%)$ & 10.77 & 11.66 & 15.93 & 15.52 & 12.51 & 15.07 & 13.71 & 12.24 \\
\hline $\min \overline{\left|\varepsilon_{1}\right|}(\%)$ & 1.86 & 4.18 & 4.02 & 5.31 & 6.95 & 7.26 & 5.16 & 6.89 \\
\hline
\end{tabular}




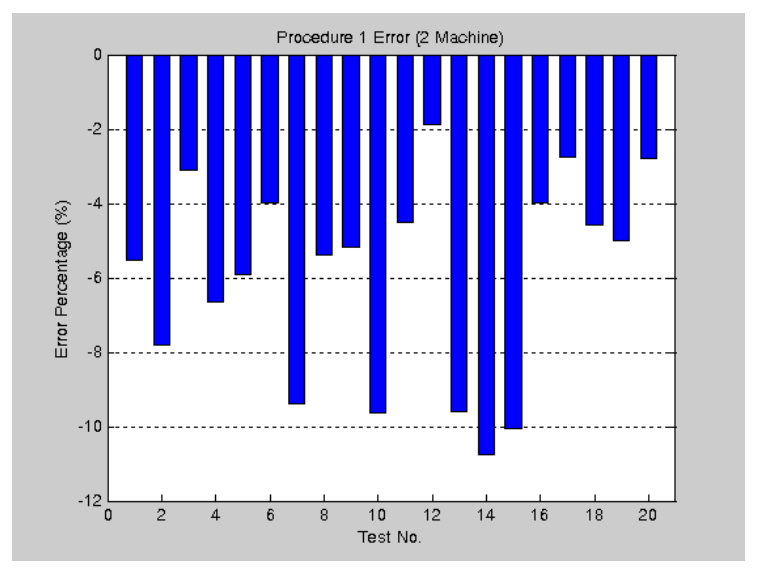

(a) 2-machine Line

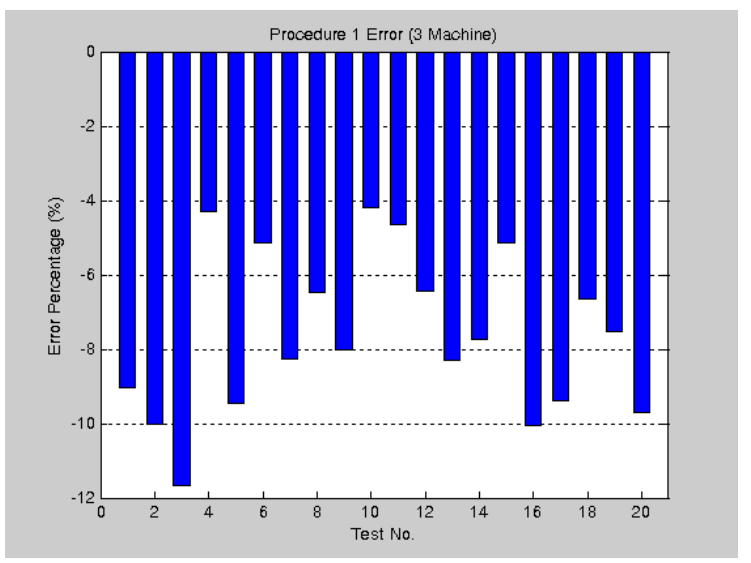

(b) 3-machine Line

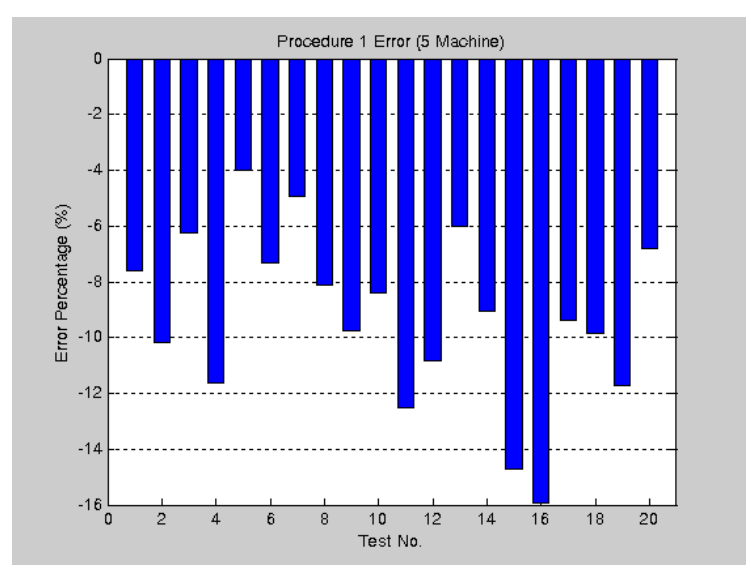

(c) 5-machine Line

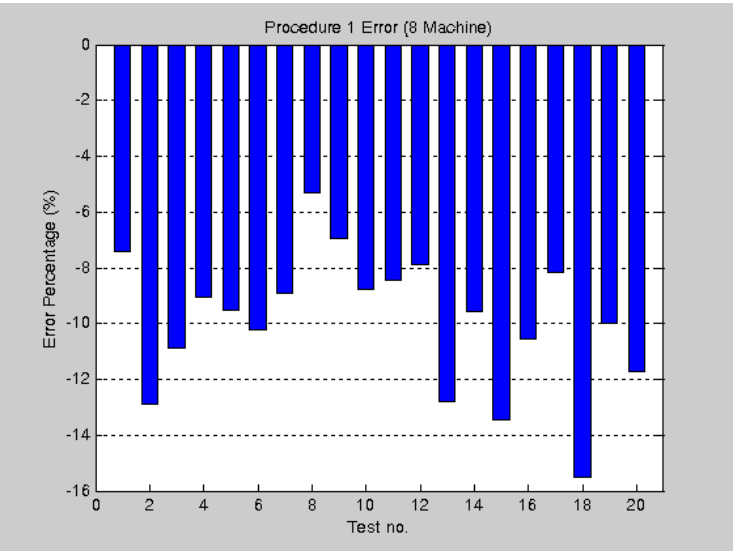

(d) 8-machine Line

Figure 4.6 Error of Procedure 1 


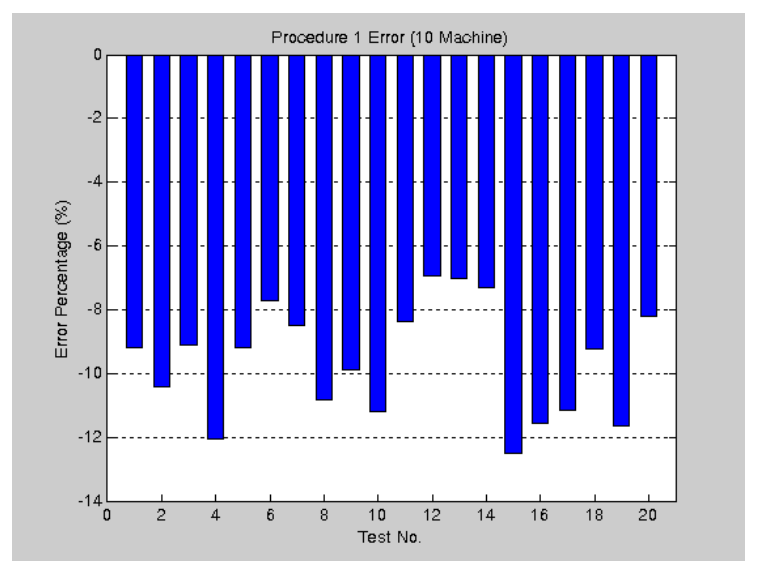

(e) 10-machine Line

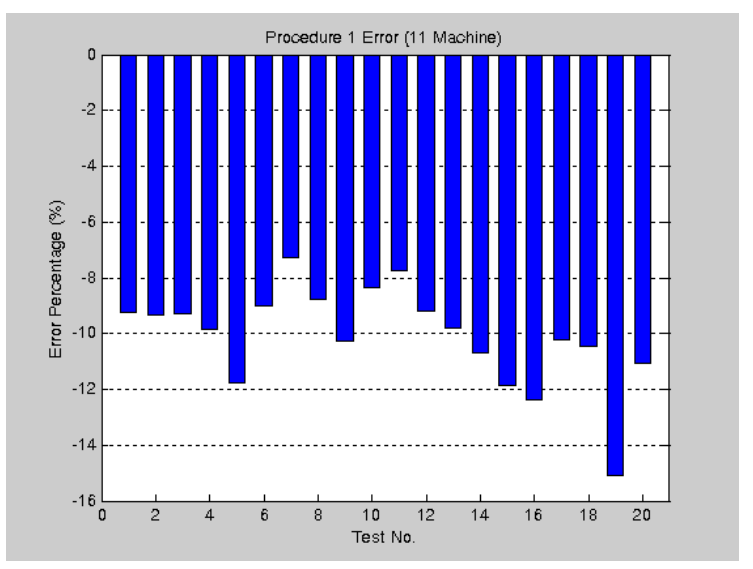

(f) 11-machine Line

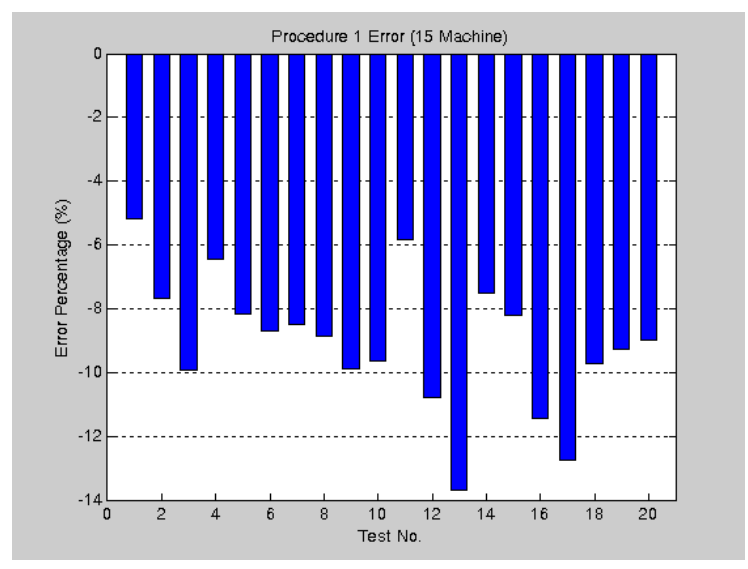

(g) 15-machine Line

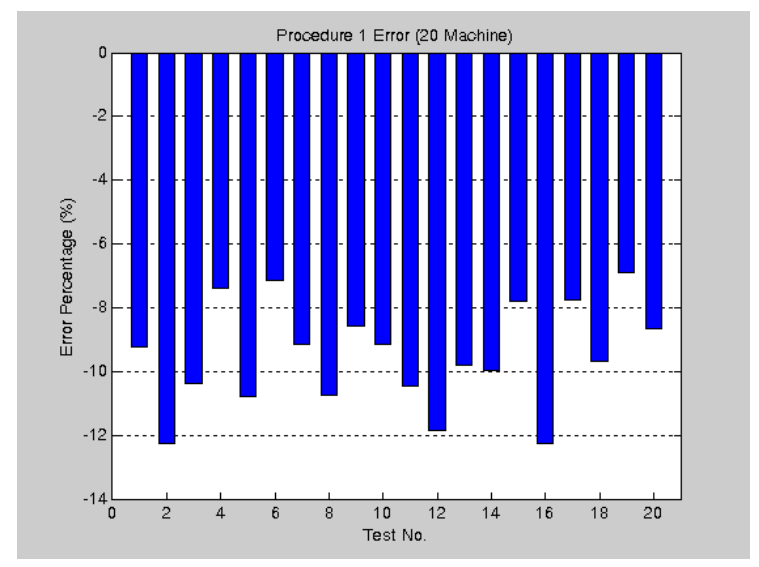

(h) 20-machine Line

Figure 4.6 Error of Procedure 1(Continued)

\subsubsection{Modified recursive procedure}


Procedure 1 presents a lower bound for performance evaluation (which may due to that assumptions 1)-7) define a block before service model, i.e., parts will not be loaded if a machine is blocked). In order to improve its accuracy, we modified the iterative equations by using $N_{i}+1$ instead of $N_{i}$. As a result, it provides higher estimation of system $P R$. The modified recursive procedure is presented below:

\section{Procedure 2.}

$$
\begin{gathered}
p_{i}^{\prime}(s+1)=p_{i}-\operatorname{pr}(s), i=1, \ldots, M, \\
\operatorname{pr}(s+1)=\operatorname{PR}\left(p_{1}^{\prime}(s+1), \ldots, p_{M}^{\prime}(s+1), p_{1}, \ldots, p_{M}, N_{11}+1, \ldots,\right. \\
\left.N_{1, M-1}+1, N_{0}+1, N_{21}+1, \ldots, N_{2, M-1}+1\right), \\
s=0,1,2, \ldots, \\
\operatorname{pr}(0)=\operatorname{PR}\left(p_{1}, \ldots, p_{M}, p_{1}, \ldots, p_{M}, N_{11}+1, \ldots, N_{1, M-1}+1, N_{0}+1, N_{21}+1, \ldots, N_{2, M-1}+1\right) .
\end{gathered}
$$

Similar to Procedure 1, the convergence of the modified recursive procedure and the uniqueness of the solution still hold.

Theorem 4.2: Under assumptions 1)-7), recursive Procedure 2 is convergent, therefore, the following limit exists:

$$
\lim _{s \rightarrow \infty} \operatorname{pr}(s):=\widehat{P R}_{2} .
$$

In addition, the steady state equations of (4.15) has a unique solution.

Proof: Similar to the proofs for Theorem 1 and Corollary 1.

Therefore, an estimate of the steady state production rate of the system, $\widehat{P R}_{2}$, is obtained. The accuracy of this estimate is again investigated numerically 
using the same lines generated from (4.13). Similarly, we introduce

$$
\varepsilon_{2}=\frac{\widehat{P R_{2}}-P R}{P R} \cdot 100 \% .
$$

The results of the analysis are shown in Figure 4.7. Clearly, the new procedure provides more closed estimation of system production rate. Table 2 presents the measurement of discrepancy of the estimates. It is shown that $\varepsilon_{2}$ ranges typically within $5-10 \%$. Considering that the data collected on the factory floor usually has 5 to $10 \%$ error, Procedure 2 provides an acceptable accuracy of system production rate estimation.

Table 2 Accuracy of Procedure 2

\begin{tabular}{|c|c|c|c|c|c|c|c|c|}
\hline $\begin{array}{c}\text { No. of } \\
\text { Machines }\end{array}$ & 2 & 3 & 5 & 8 & 10 & 11 & 15 & 20 \\
\hline$\overline{\left|\varepsilon_{2}\right|}(\%)$ & 1.79 & 2.03 & 2.44 & 3.62 & 3.71 & 5.08 & 5.83 & 7.26 \\
\hline $\max \overline{\left|\varepsilon_{2}\right|}(\%)$ & 4.59 & 5.54 & 7.13 & 9.05 & 8.34 & 11.57 & 10.57 & 11.96 \\
\hline $\min \overline{\varepsilon_{2} \mid}(\%)$ & 0.12 & 0.31 & 0.07 & 0.10 & 0.89 & 0.60 & 1.60 & 2.01 \\
\hline
\end{tabular}




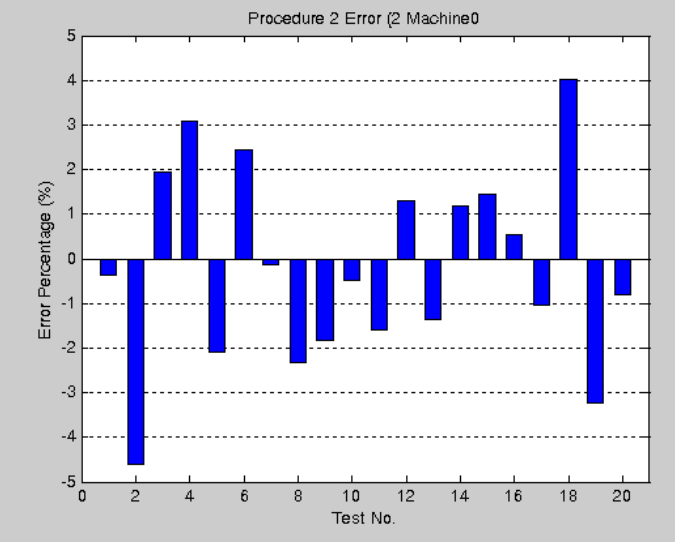

(a) 2-machine Line

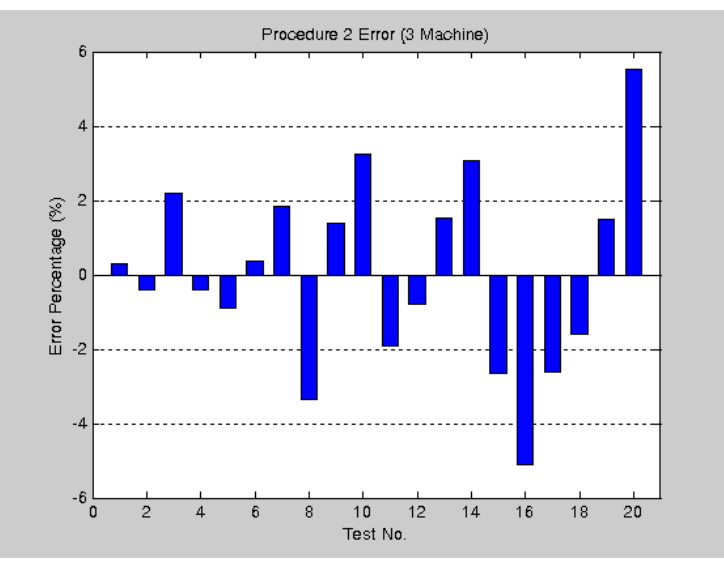

(b) 3-machine Line

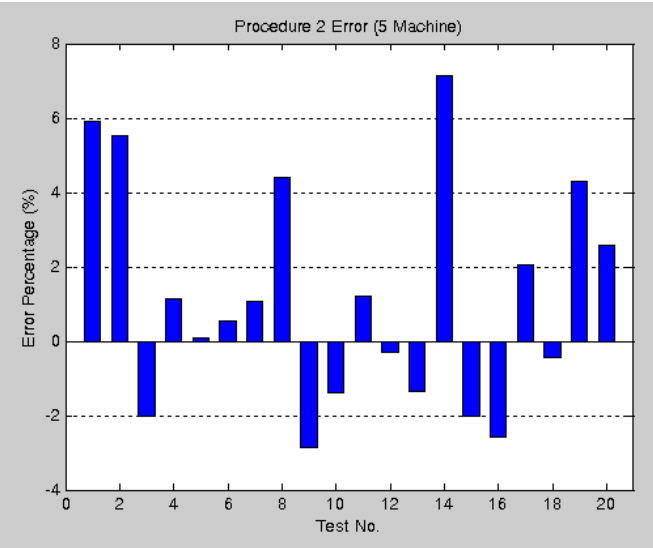

(c) 5-machine Line

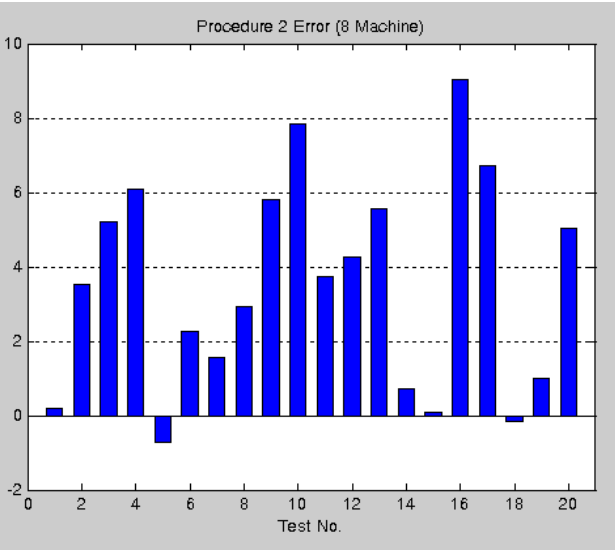

(d) 8-machine Line

Figure 4.7 Error of Procedure 2 


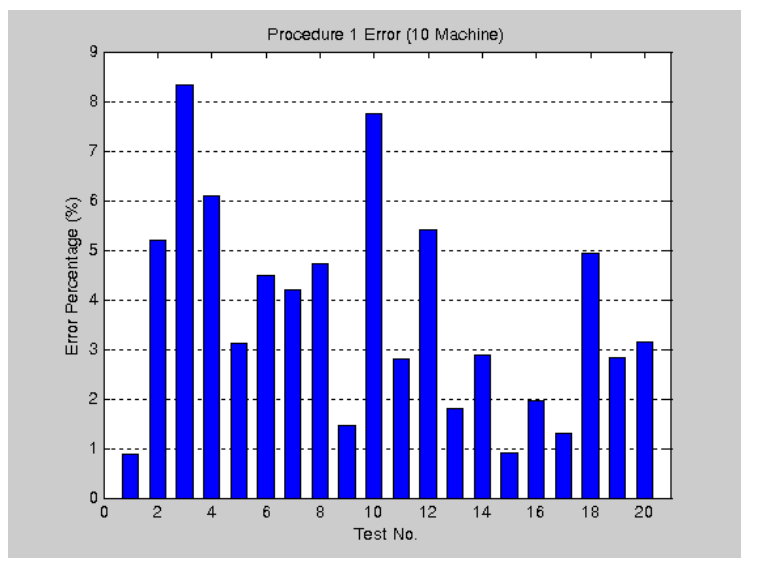

(e) 10-machine Line

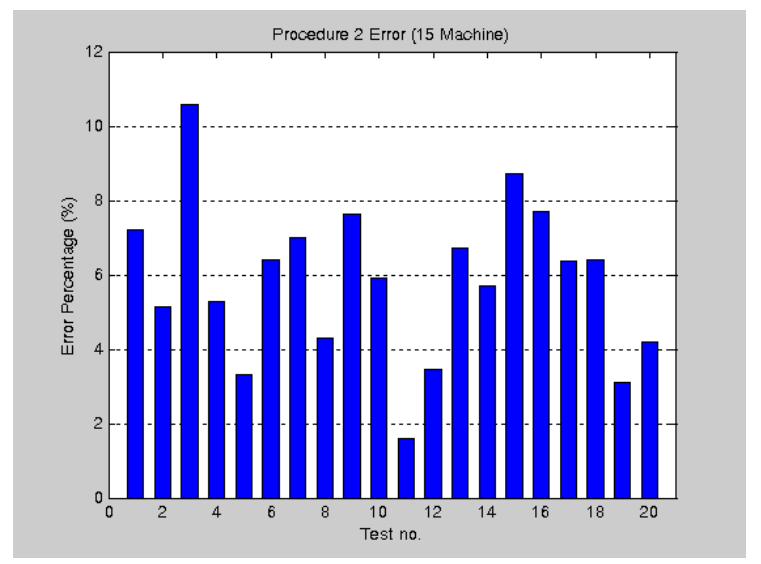

(g) 15-machine Line

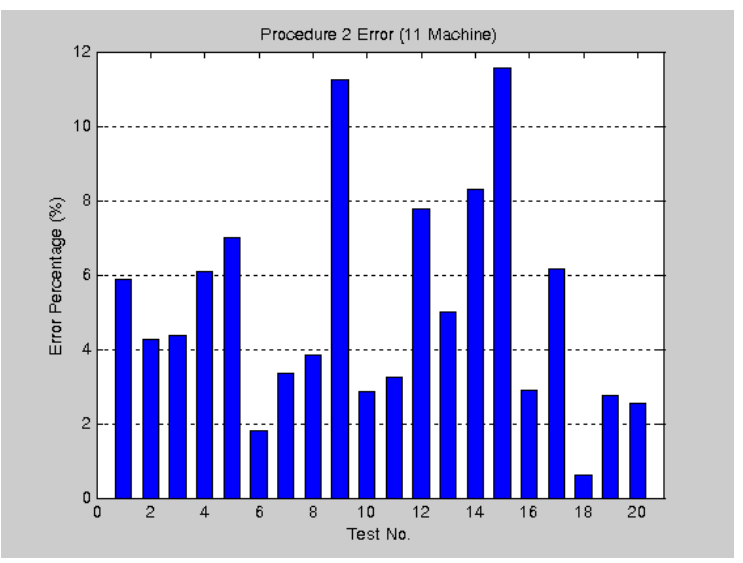

(f) 11-machine Line

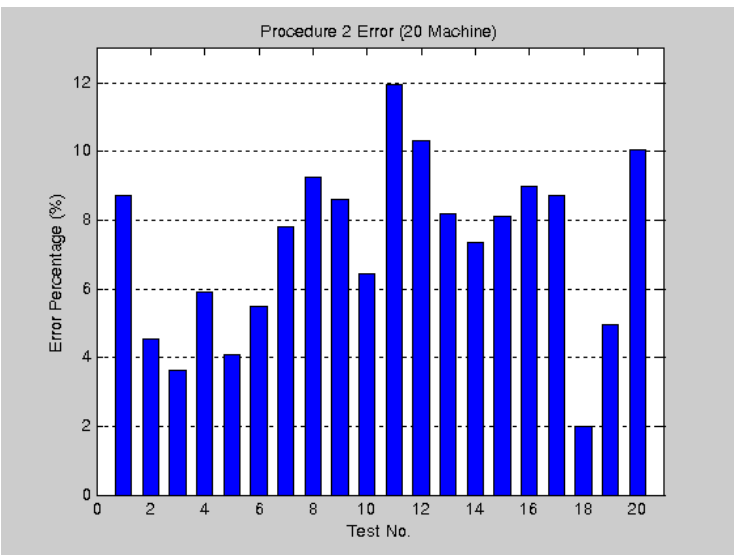

(h) 20-machine Line

Figure 4.7 (Continued) Error of Procedure 2

Remark 3 : By using the serial line analysis operator $P R(\cdot)$ in Procedures 1 and 
2, we can also obtain the work-in-process $(W I P)$ of the system for buffers $N_{i j}$, $i=1,2, j=1, \ldots, M$, and $N_{0}$ using (4.6) and (4.7) (see [8] for details). Applying the Little's law:

$$
W I P=P R \cdot \text { Flow Time }
$$

the flow time (or cycle time in semiconductor industry) can be calculated.

\subsection{Extensions}

With minor changes, Procedures 1 and 2 can be extended to other reentrant lines other than two layer LBFS ones. Here we study re-entrant lines with different machine parameters for $1^{\text {st }}$ and $2^{\text {nd }}$ time jobs, and multiple layer reentrant lines.

\subsubsection{Re-entrant lines with different machine parameters for $1^{\text {st }}$ and $2^{\text {nd }}$ time jobs}

\section{A) Analytical expressions}

In some re-entrant systems, machines may have different parameters (e.g., processing rates, efficiencies, etc.) for the first and second time jobs. As it is shown in Figure 4.8, each machine has two parameters $p_{1 i}$ and $p_{2 i}$, $i=1, \ldots, M$, corresponding to the first and second time jobs, respectively. Clearly, Procedures 1 and 2 can be applied to such systems as well. In this case, the resulting $2 M$-machine serial line will have parameters $p_{1 i}^{\prime}=p_{1 i}-p r, i=1, \ldots, M$, for first $M$ machines, and $p_{2 i}$ for latter $M$ machines (see Figure 4.9, where, as before, $p r$ can be solved from Procedures 1 and 2), i.e., the first equations are 
changed to $p_{i}^{\prime}(s+1)=p_{1 i}-\operatorname{pr}(s), i=1, \ldots, M$. In other words, the following equation is used in the iteration procedure:

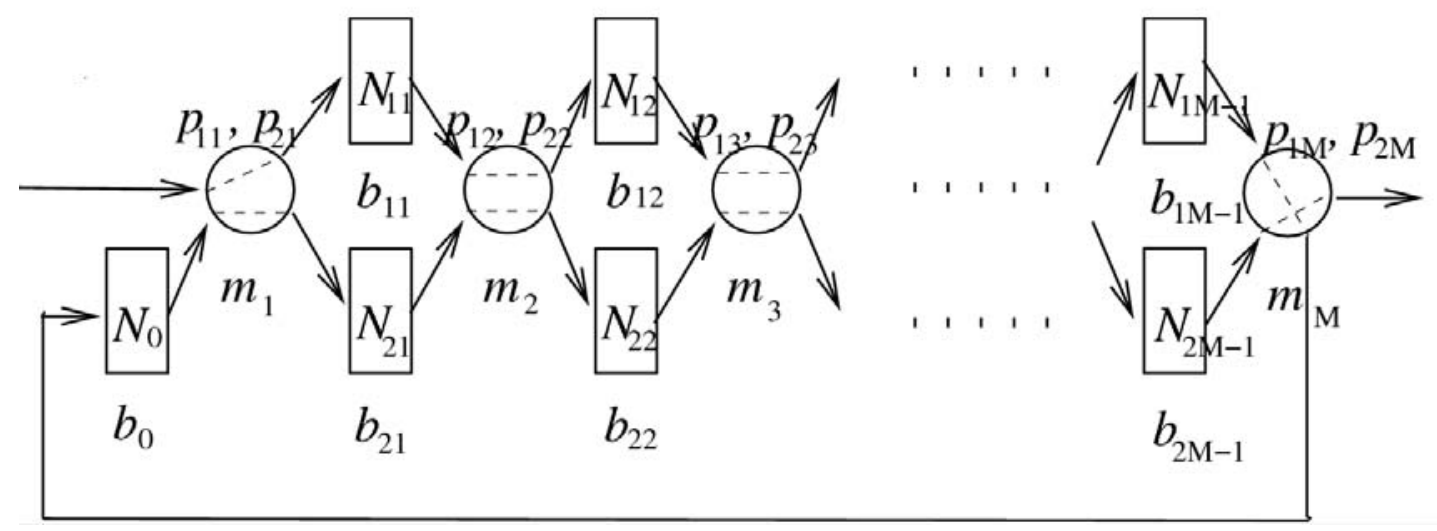

Figure 4.8 Re-entrant Line with Different Machine Parameters for 1st and 2nd Time Jobs

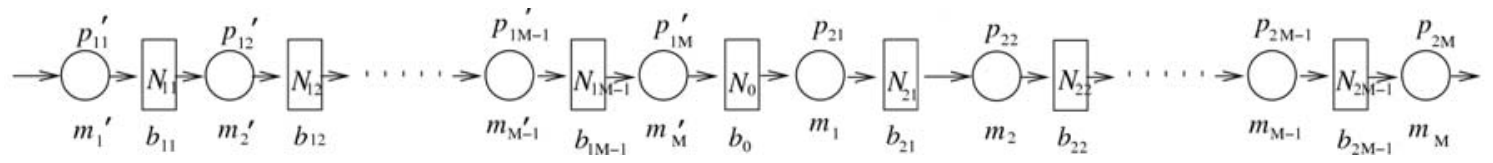

Figure 4.9 Equivalent Serial Line

$$
\begin{gathered}
\operatorname{pr}(s+1)=\operatorname{PR}\left(p_{11}-\operatorname{pr}(s), \ldots, p_{1 M}-\operatorname{pr}(s), p_{21}, \ldots, p_{2 M},\right. \\
\left.N_{11}, \ldots, N_{1, M-1}, N_{0}, N_{21}, \ldots, N_{2, M-1}\right), \\
s=0,1,2, \ldots
\end{gathered}
$$

Similar changes can be applied to the modified recursive procedure, i.e.,

$$
\begin{gathered}
\operatorname{pr}(s+1)=P R\left(p_{11}-\operatorname{pr}(s), \ldots, p_{1 M}-\operatorname{pr}(s), p_{21}, \ldots, p_{2 M},\right. \\
\left.N_{11}+1, \ldots, N_{1, M-1}+1, N_{0}+1, N_{21}+1, \ldots, N_{2, M-1}+1\right), \\
s=0,1,2, \ldots
\end{gathered}
$$

The convergence of the procedures, uniqueness of solution can be proved 
analogously.

Theorem 4.3: Under assumptions 1)-7), for re-entrant lines with different machine parameters, Procedure 1 and 2 are convergent and unique solution exists:

Proof: Similar to the proof of Theorem 4.1 and 4.2.

In addition, numerical experiments are conducted to verify the accuracy and the results are presented below.

\section{B) Accuracy}

The accuracy of the approximation is investigated numerically using similar approach introduced before. Again, we consider M-machine re-entrant lines, where $M \in\{2,5,8,10,11,15,20\}$. For each $M$, we construct 20 lines by randomly and equiprobably selecting machine and buffer parameters from (4.13). However, now $p_{1 i}$ and $p_{2 i}$ are selected independently and different. As a result, a total of 140 re-entrant lines are investigated. Procedures 1 and 2 are used for analytical calculation and simulations are carried out for justification purpose. The differences between analytical and simulation results are evaluated as:

$$
\varepsilon_{3}=\frac{\widehat{P R_{3}}-P R}{P R} \cdot 100 \%,
$$


where $P R$ and $\widehat{P R}_{3}$ are the production rates obtained by simulation and recursive procedure, respectively. The results of this investigation are illustrated in Figure 4.10.

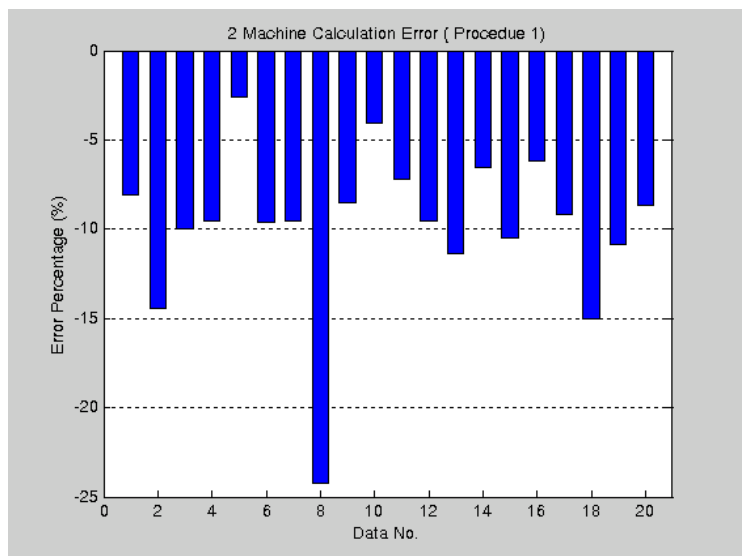

(a) 2-machine Line (Procedure 1)

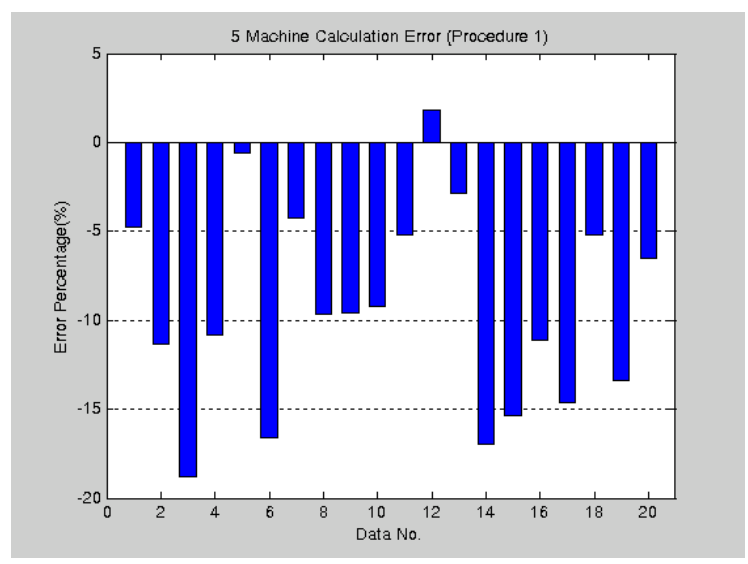

(c) 5-machine Line (Procedure 1)

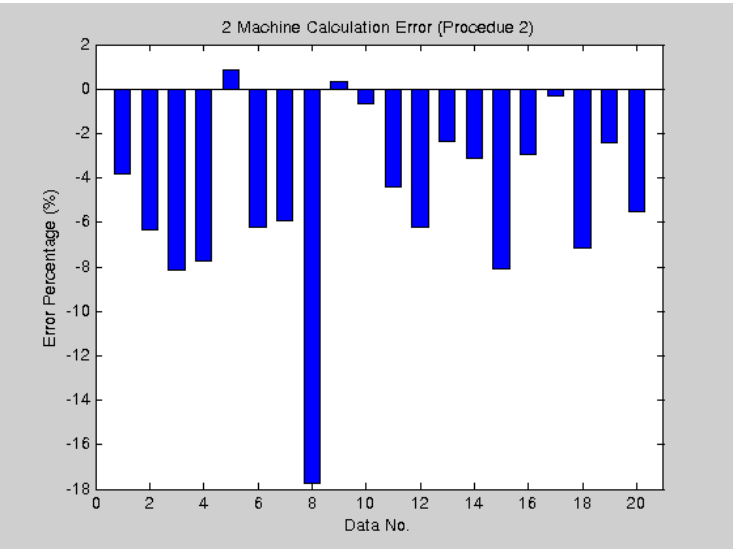

(b) 2-machine Line (Procedure 2)

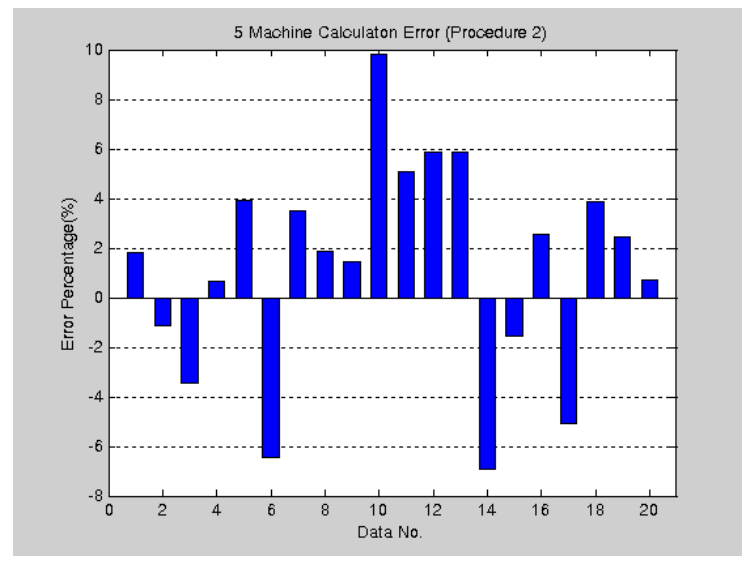

(d) 5-machine Line (Procedure 2)

Figure 4.10 Error of Procedure 2 for Re-entrant Lines with Different Processing Parameter of Jobs 


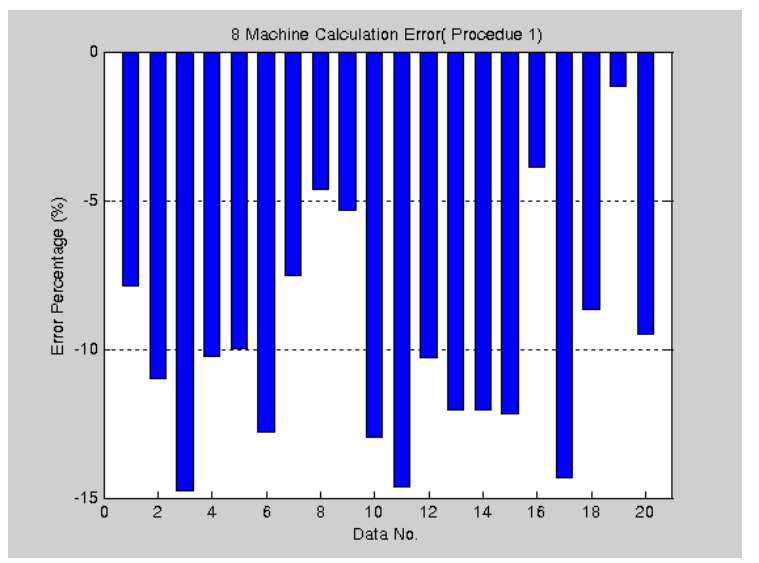

(e) 8-machine Line (Procedure 1)

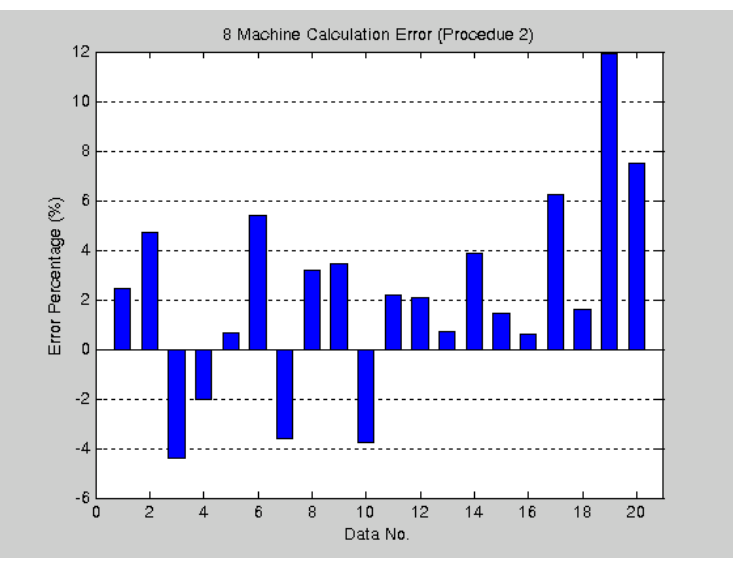

(f) 8-machine Line (Procedure 2)

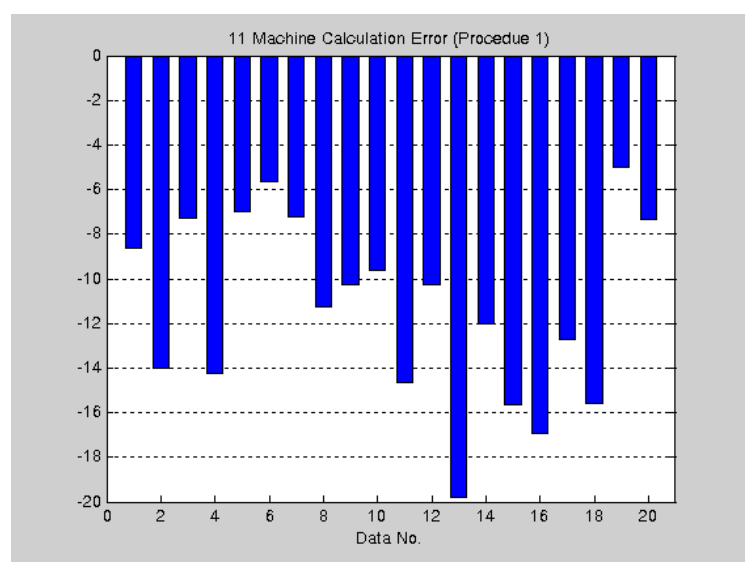

(g) 11-machine Line (Procedure 1)

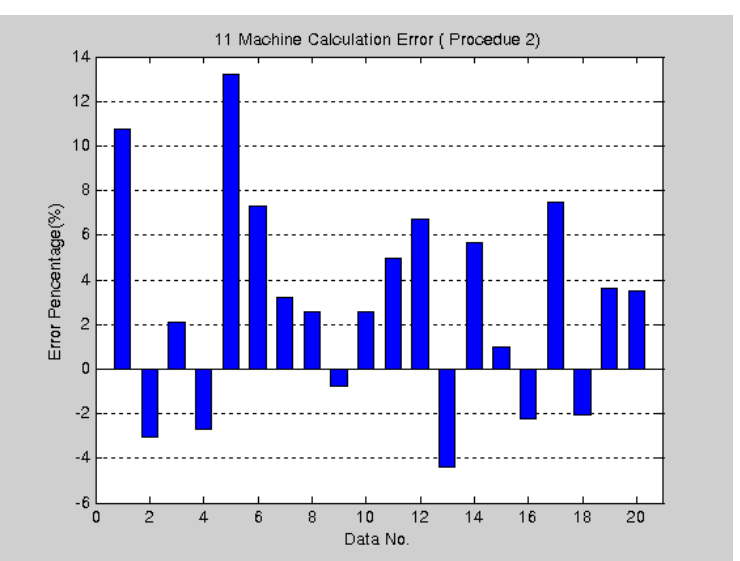

(h) 11-machine Line (Procedure 2)

Figure 4.10 (continued) Error of Procedure 2 for re-entrant lines with different processing parameter of jobs 


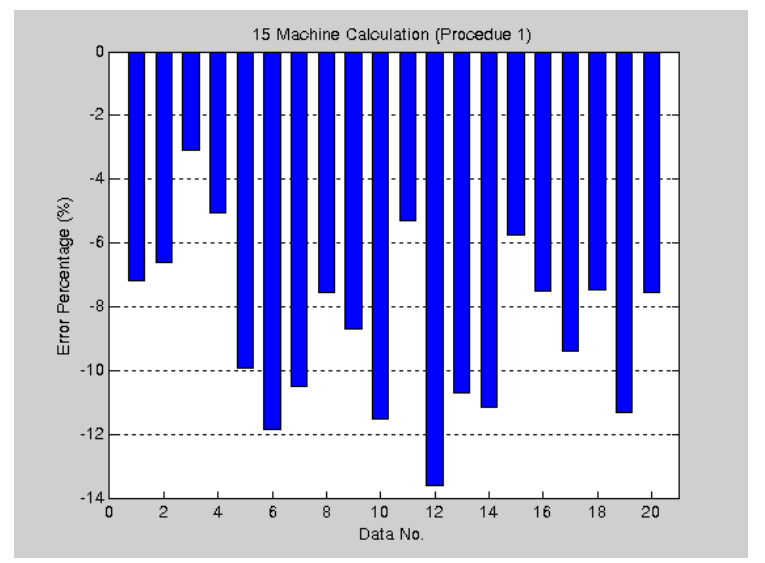

(i) 15-machine Line (Procedure 1)

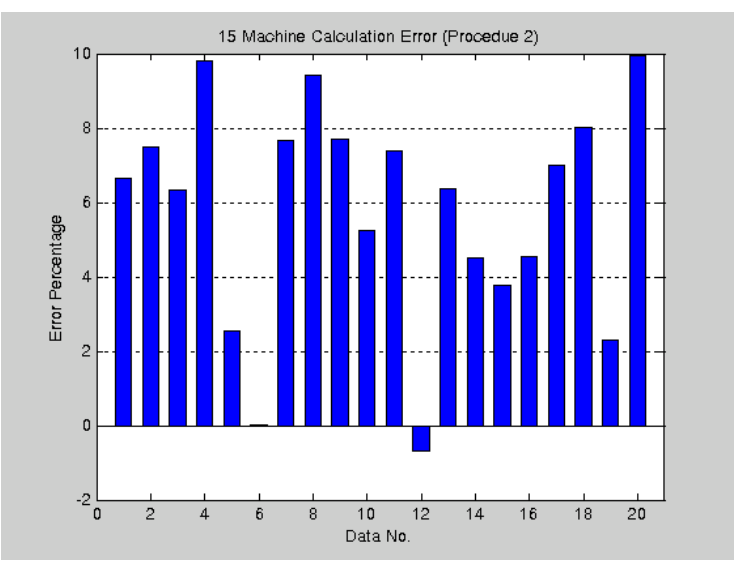

(j) 15-machine Line (Procedure 2)

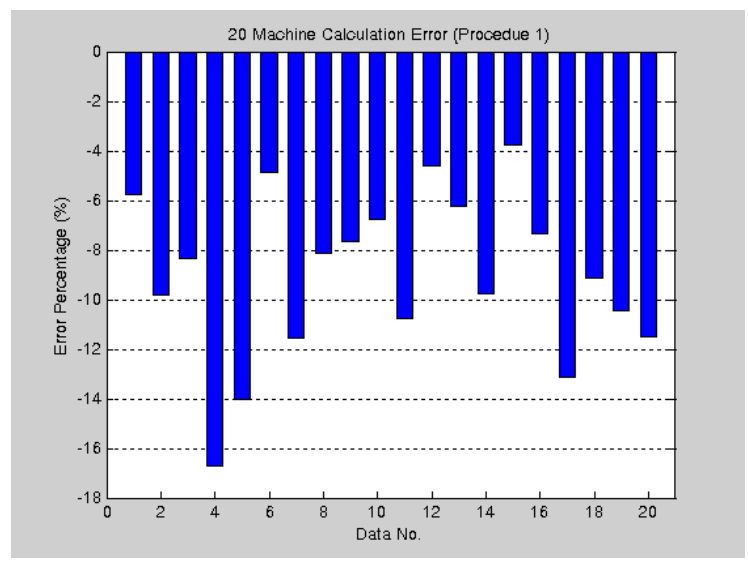

(k) 20-machine Line (Procedure 1)

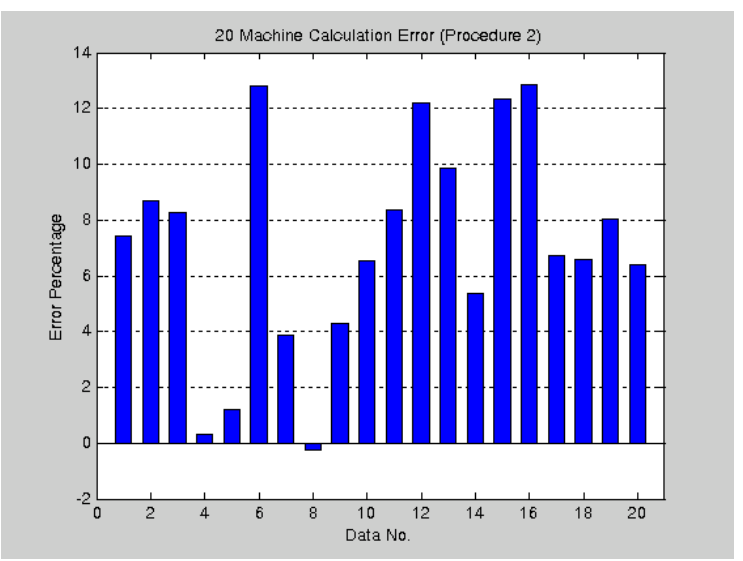

(I) 20-machine Line (Procedure 2)

Figure 4.10 (continued) Error of Procedure 2 for re-entrant lines with different processing parameter of jobs

It is shown that in all the cases we studied, Procedure 1 provides a lower bound for production rate estimation. The bound is tighter for shorter lines, and 
the average discrepancy is typically within 0 to $-15 \%$. For Procedure 2, the error is typically within $\pm 10 \%$.

\subsubsection{Re-entrant lines with more than two times of processing jobs}

In many re-entrant lines, jobs may be processed more than two times. To avoid messy notations and for simplicity, here we use a three-time processing reentrant line, shown in Figure 4.11, as an example. The general $k$-time-processing re-entrant lines can be analyzed similarly. Typically, the priority rule is applied, i.e., parts have been processed more would have higher priority. In this case, Procedures 1 and 2 can be extended. Again we approximate such lines using equivalent serial production lines. The equivalent serial line of re-entrant line in Figure 4.11 is illustrated in Figure 4.12, where we introduce pseudo machines $m_{i}^{\prime \prime}$ and $m_{i}^{\prime}, i=1, \ldots, M$, to denote machines dedicated to the first and second time processing of jobs, respectively, with parameters $p_{i}^{\prime \prime}=p_{i}-2 p r$ and $p_{i}^{\prime}=p_{i}-p r$, respectively. Procedure 1 is then modified as

$$
\begin{gathered}
\operatorname{pr}(s+1)=\operatorname{PR}\left(p_{1}-2 \operatorname{pr}(s), \ldots, p_{M}-2 \operatorname{pr}(s), p_{1}-2 \operatorname{pr}(s), \ldots, p_{M}-2 \operatorname{pr}(s),\right. \\
p_{1}-\operatorname{pr}(s), \ldots, p_{M}-\operatorname{pr}(s), p_{1}, \ldots, p_{M}, N_{11}, \ldots, N_{1, M-1}, N_{01} \\
\left.N_{21}, \ldots, N_{2, M-1}, N_{02}, N_{31}, \ldots, N_{3, M-1}\right) .
\end{gathered}
$$

Again, Procedure 2 can be modified accordingly as

$$
\begin{gathered}
\operatorname{pr}(s+1)=\operatorname{PR}\left(p_{1}-2 \operatorname{pr}(s), \ldots, p_{M}-2 \operatorname{pr}(s), p_{1}-2 p r(s), \ldots, p_{M}-2 p r(s),\right. \\
p_{1}-\operatorname{pr}(s), \ldots, p_{M}-\operatorname{pr}(s), p_{1}, \ldots, p_{M}, N_{11}+1, \ldots, N_{1, M-1}+1, N_{01}+1 \\
\left.N_{21}+1, \ldots, N_{2, M-1}+1, N_{02}+1, N_{31}+1, \ldots, N_{3, M-1}+1\right) .
\end{gathered}
$$




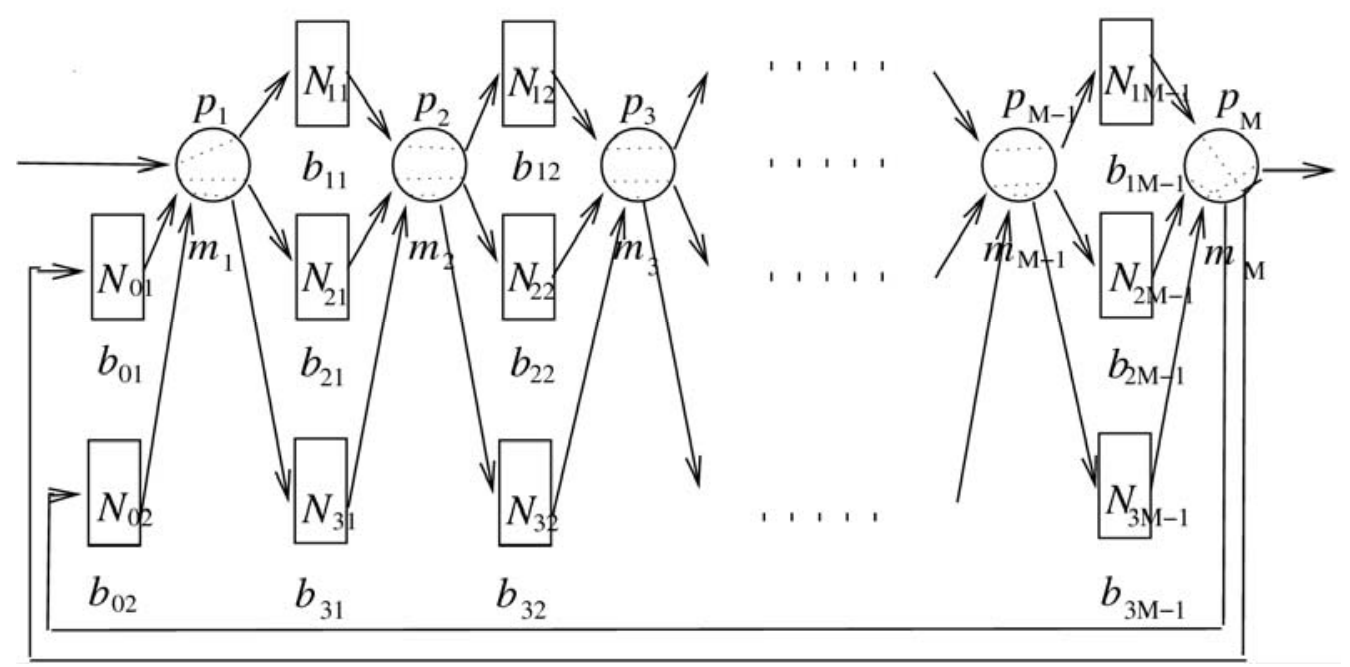

Figure 4.11 Re-entrant Line with Three-time-processing of Jobs

Clearly, this approach is also applicable to the case that machines have different parameters for the first, second, and third time processing of jobs. Analogously, the convergence of the procedures, and uniqueness of the solution can be proved analytically.

Theorem 4.4: Under assumptions 1)-7), for re-entrant lines with more than two times of jobs, Procedure 1 and 2 are convergent and unique solution exists.

Proof: Similar to the proof of Theorems 4.1 and 4.2.

Using the parameters defined in (4.13), 20 four-machine lines have been generated randomly and equiprobably. Simulations are carried out to evaluate the accuracy. The resulting errors are shown in Figure 4.13. Again, Procedure 1 
provides a lower bound with tightness typically within $10 \%$, and Procedure 2 has higher accuracy, the errors are usually less than $5 \%$.

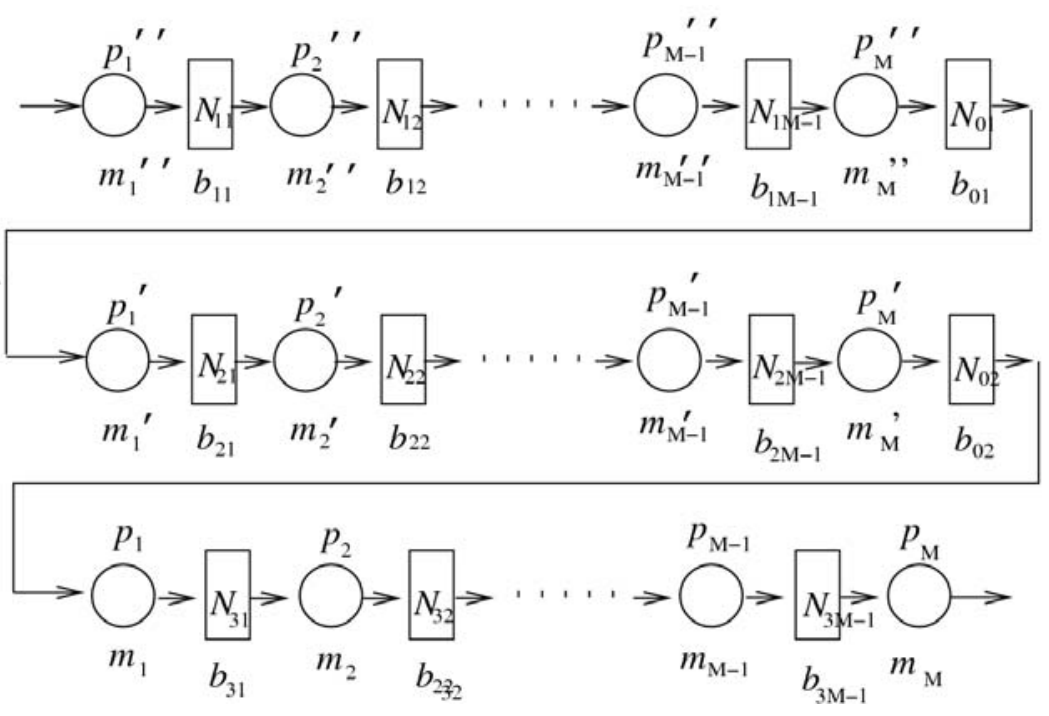

Figure 4.12 Equivalent Serial Line of Line in Figure 4.11

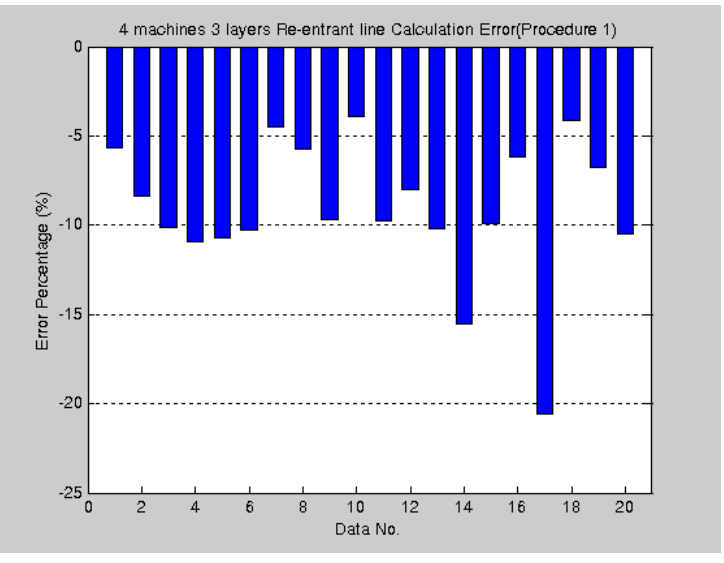

(a) Procedure 1

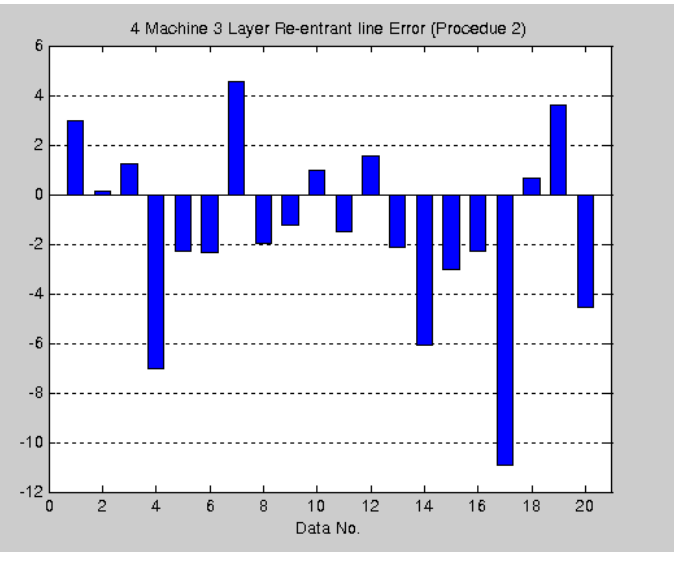

(b) Procedure 2

Figure 4.13 Errors for 4-machine, 3-Layer Re-entrant Line using Procedures 1 and 2 


\section{CHAPTER 5}

\section{STRUCTURAL PROPERTIES}

\subsection{Asymptotic Properties}

It has been shown in [8] that for serial lines with Bernoulli machines, when buffer capacity $N$ goes to infinity, the production rate converges to $\min \left(p_{1}, p_{2}, \ldots\right.$, $\left.p_{M}\right)$. Similarly, we can prove that the asymptotic properties of re-entrant lines.

Theorem 5.1: Under assumptions 1)-7),

$$
\lim _{N_{i} \rightarrow \infty, \forall i} P R=\min \left(p_{1}, p_{2}, \ldots, p_{M}\right) / 2 .
$$

Proof: See Appendix.

Figure 5.1 shows the numerical test of $P R$ as a function of buffer size $N$ for a three-machine re-entrant line using the following parameters with identical buffer capacity $N_{i}, i=0,1, \ldots, 5$.

$$
p_{1}=0.9, p_{2}=0.7, p_{3}=0.8
$$

We can see that as buffer $N$ increases, $P R$ is increasing with a decreasing rate, and when buffer size increases to 18 , which is fairly large for this line, $P R$ approaches $0.35=p_{2} / 2$.

\subsection{Monotonicity}

It has been shown in [8] that monotonicity holds in serial lines and assembly systems, i.e., improving machine reliability and/or increasing buffer capacity lead to improvement of system production rate. Similar properties are 
observed in re-entrant lines as well.

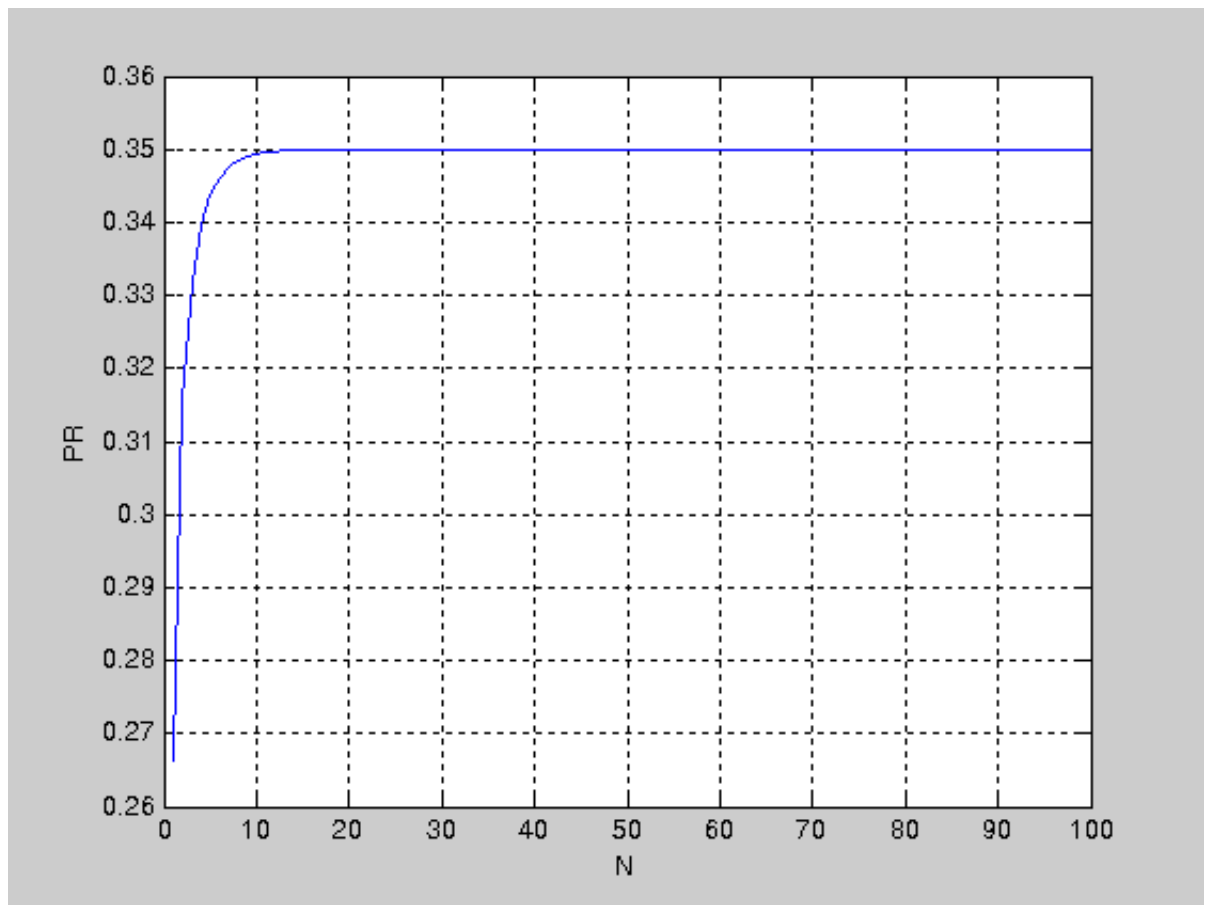

Figure 5.1 PR as Function of Buffer Capacity

Theorem 5.2: Under assumptions 1)-7), the system production rates are monotonically increasing with respect to $p_{i}, i=1, \ldots, M$, and $N_{i}, i=0,1, \ldots, M-1$. Proof: See Appendix.

Figure 5.1 can also be used to illustrate monotonicity with respect to $N$. Figure 5.2 gives an example of this monotonicity property with respect to $p$ for a three- machine re-entrant line using Procedure 2. The machine parameters are given as follows:

$$
N_{i}=5, \quad i=0,1, \ldots, 5 \text {, }
$$




$$
p_{1}=p_{3}=0.6
$$

and $p_{2}$ is increasing from 0 to 1 . As is shown in the Figure, $P R$ increases with $p_{2}$, however, when $p_{2}>0.6$, the increasement has a decreasing rate.

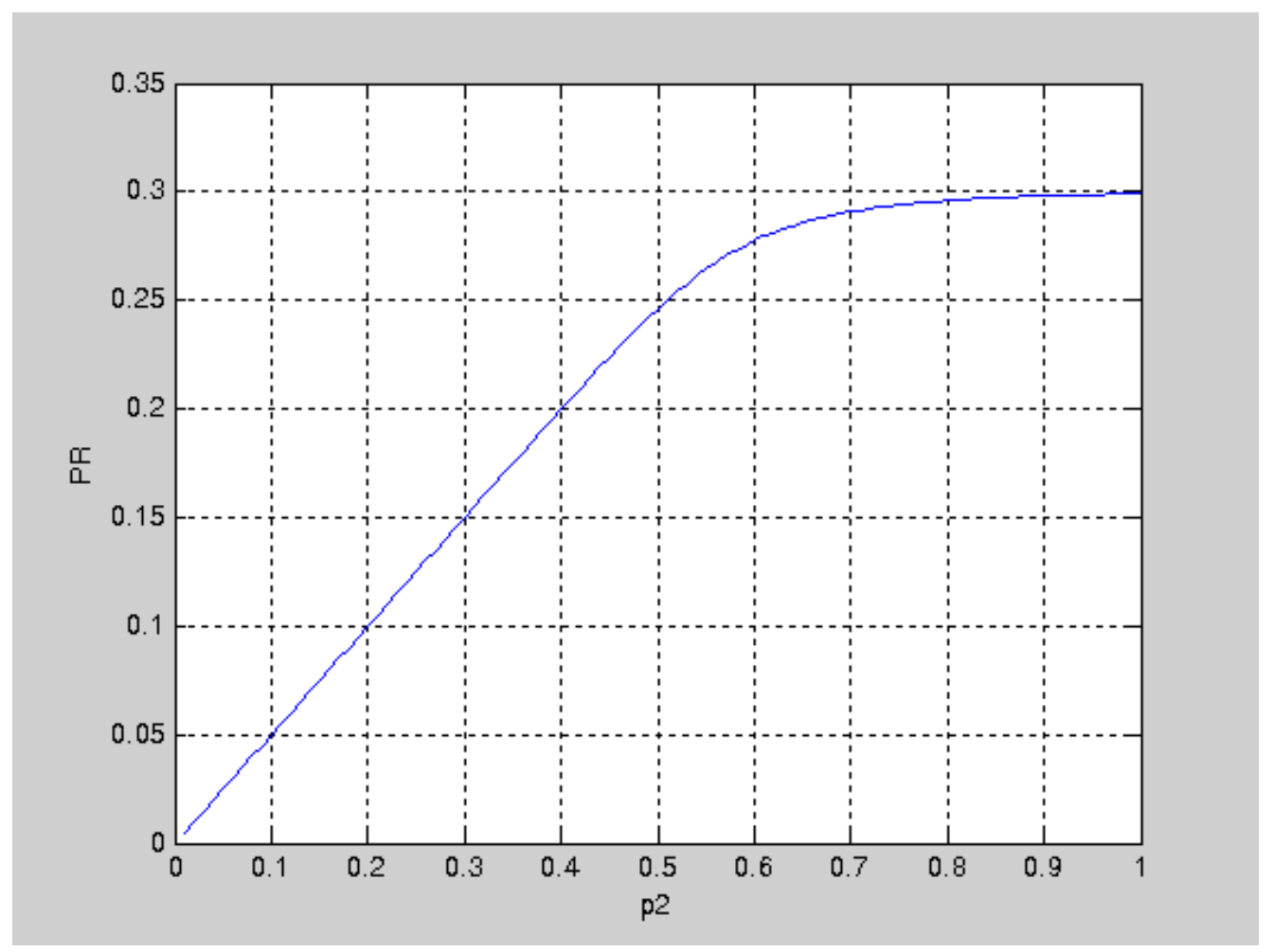

Figure 5.2 $P R$ as Function of $p_{2}$

\subsection{Reversibility}

Reversibility is observed in serial production lines as well ([8]). For reentrant lines, reversibility is understood in the following sense: Consider the reentrant line described in Figure 3.1, the reversed line is shown in Figure 5.3. The priority is again assigned to buffer $b_{2 i}, i=1, \ldots, M-1$. Let $\widehat{P R}_{i}$ and $\widehat{P R}_{i}^{\text {rev }}$ denote the production rates obtained for Procedure $i, i=1,2$, for the original and 
reversed lines, respectively. Then we have

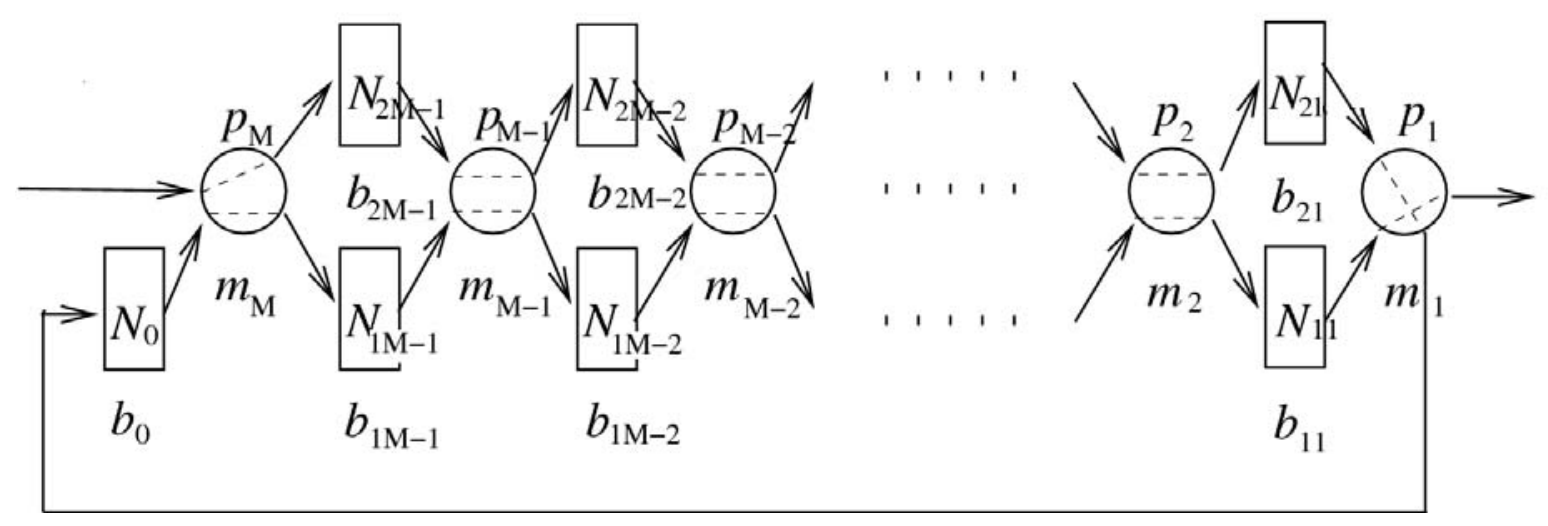

Figure 5.3 Reversed Re-entrant Lines

Theorem 5.3: Under assumptions 1)-7),

$$
\widehat{P R}_{i}=\widehat{P R}_{i}^{\text {rev }}, \quad i=1,2 .
$$

Proof: See Appendix.

\subsection{Policy Comparison between FBFS and LBFS.}

The procedures developed in Chapter 4 are based on Last Buffer First Serve (LBFS) policy, which prioritizes the second time jobs. In addition to LBFS, another policy, First Buffer First Serve (FBFS) policy, is also studied, with which the priority is given to first time jobs. In such systems, assumptions 1)-7) still hold with the only exception that FBFS is used. Here we define assumption 6') as:

6') The first time jobs have higher priorities than the second time ones. In other words, machine $m_{i}, i=2, \ldots, M-1$, always takes a part from buffer $b_{1, i-1}$ if it is 
not empty and $m_{i}$ is not blocked by $b_{1}$, otherwise it will take a part from buffer $b_{2, \mathrm{i}-1}$ if it is not empty and $m_{i}$ is not blocked by $b_{2 i}$. Machine $m_{1}$ takes a new part if is not blocked by $b_{11}$, otherwise it will take a part from buffer $b_{0}$ if it is not blocked by $b_{21}$. Machine $m_{M}$ will take part from $b_{1 M-1}$ if it is not empty, otherwise $m_{M}$ loads from $b_{2, M-1}$ if it is not empty and complete one part.

Therefore, we can adopt similar procedures in Chapter 4 to study FBFS policy re-entrant lines.

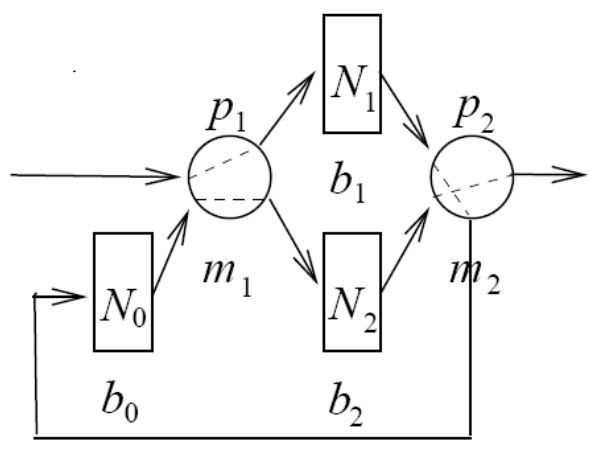

Figure 5.4 Two-machine Re-entrant Line with FBFS Policy

Figure 5.4 depicts a two-machine line with FBFS policy. Because of FBFS policy, the priority is with first time jobs. The availability of $m_{2}$ to first time job is when $m_{2}$ is up and not blocked by buffer $N_{0}$. The availability of $m_{2}$ to second time jobs is when $m_{2}$ is up but could not process first time jobs. It is equivalent that a machine with reliability $p_{2}-p r_{2}^{(1)}$ is available to second time jobs. Similarity, machine 1 can also be analyzed accordingly. Thus we can equalize the twomachine re-entrant line with FBFS policy using the four-machine serial line in Figure 5.5. The production rate of this serial line can be approximated using 
recursive Procedures 1 and 2 discussed in Chapter 4 with minor changes, where the differences are the sequences of the serial line machines. With FBFS, $p_{1}$ through $p_{M}$ come first, and $p_{1-p r}$ through $p_{M-}-p r$ follow, while with LBFS $p_{1-p r}$ through $p_{M-}$ pr come first, and $p_{1}$ through $p_{M}$ next.

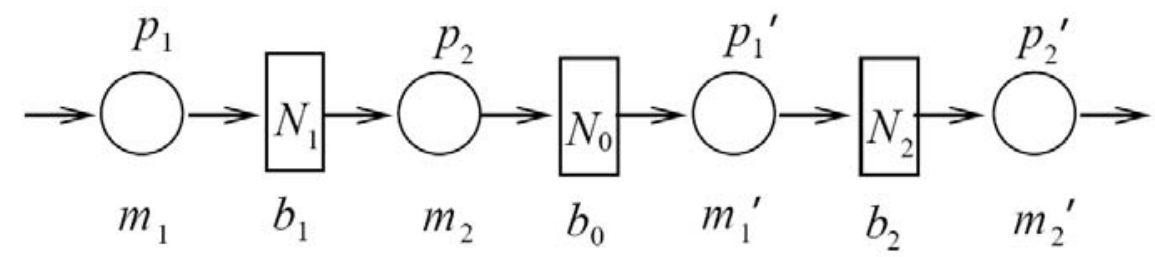

Figure 5.5 Equivalent Serial Line of the Re-entrant Line in Figure 5.4 Procedure 3 (FBFS):

$$
\begin{aligned}
& p_{i}^{\prime}(s+1)=p_{i}-\operatorname{pr}(s), i=1, \ldots, M, \\
& \operatorname{pr}(s+1)=\operatorname{PR}\left(p_{1}, \ldots, p_{M}, p_{1}^{\prime}(s+1), \ldots, p_{M}^{\prime}(s+1),\right. \\
& \left.N_{11}, \ldots, N_{1, M-1}, N_{0}, N_{21}, \ldots, N_{2, M-1}\right), \\
& \quad s=0,1,2, \ldots,
\end{aligned}
$$

where

$$
\operatorname{pr}(0)=P R\left(p_{1}, \ldots, p_{M}, p_{1}, \ldots, p_{M}, N_{11}, \ldots, N_{1, M-1}, N_{0}, N_{21}, \ldots, N_{2, M-1}\right) .
$$

Procedure 4 (FBFS):

$$
\begin{gathered}
p_{i}^{\prime}(s+1)=p_{i}-\operatorname{pr}(s), i=1, \ldots, M, \\
\operatorname{pr}(s+1)=\operatorname{PR}\left(p_{1}, \ldots, p_{M}, p_{1}^{\prime}(s+1), \ldots, p_{M}^{\prime}(s+1),\right. \\
\left.N_{11}, \ldots, N_{1, M-1}, N_{0}, N_{21}, \ldots, N_{2, M-1}\right), \\
s=0,1,2, \ldots,
\end{gathered}
$$

where 


$$
\operatorname{pr}(0)=P R\left(p_{1}, \ldots, p_{M}, p_{1}, \ldots, p_{M}, N_{11}+1, \ldots, N_{1, M-1}+1, N_{0}+1, N_{21}+1, \ldots, N_{2, M-1}+1\right)
$$

It is easy to show that Procedures 3 and 4 are convergent as well.

Theorem 5.4: Under assumptions 1)-5), 6'), 7), recursive procedures 3 and 4 are convergent. In addition, a unique solution exists in each procedure.

Proof: Similar to the proof of Theorem 4.2.

Moreover, the system-theoretic properties, such as asymptotic property, monotonicity, and reversibility, hold for lines with FBFS policy.

The differences in system performance between FBFS and LBFS policies can be studied using these procedures. These two policies are compared with the same production lines, WIPs are also compared to evaluate the overall performance of these two policies. To verify the result, 120 re-entrant lines are generated using the parameters from (4.13).

Figure 5.6 shows the production rate comparison between these two policies. For each of these lines, analytical method using Procedure 2 are employed to evaluate system production rate. Simulation results are also provided for comparison purpose. We define the difference between LBFS and FBFS as follows for simulation results and Procedure 2 calculations as follows:

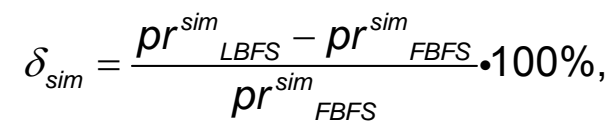




$$
\hat{\delta}=\frac{\widehat{p r}_{L B F S}-\widehat{p r}_{F B F S}}{\widehat{p r}_{F B F S}} \cdot 100 \%
$$

where $\delta_{\text {sim }}$ and $\hat{\delta}$ denote the production rate differences obtained by simulation and Procedure 2, respectively.

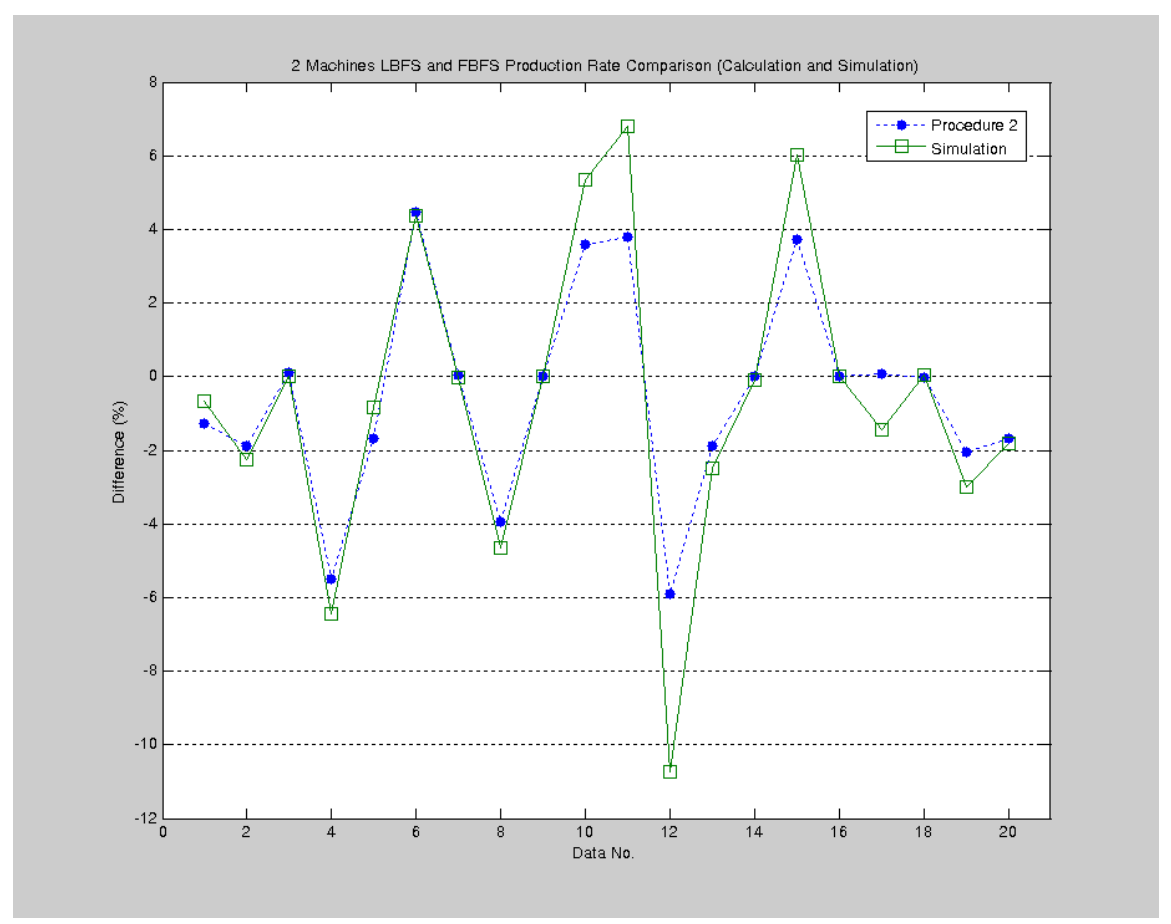

Figure 5.6 (a) PR Comparison using FBFS and LBFS (2-machine re-entrant line) 


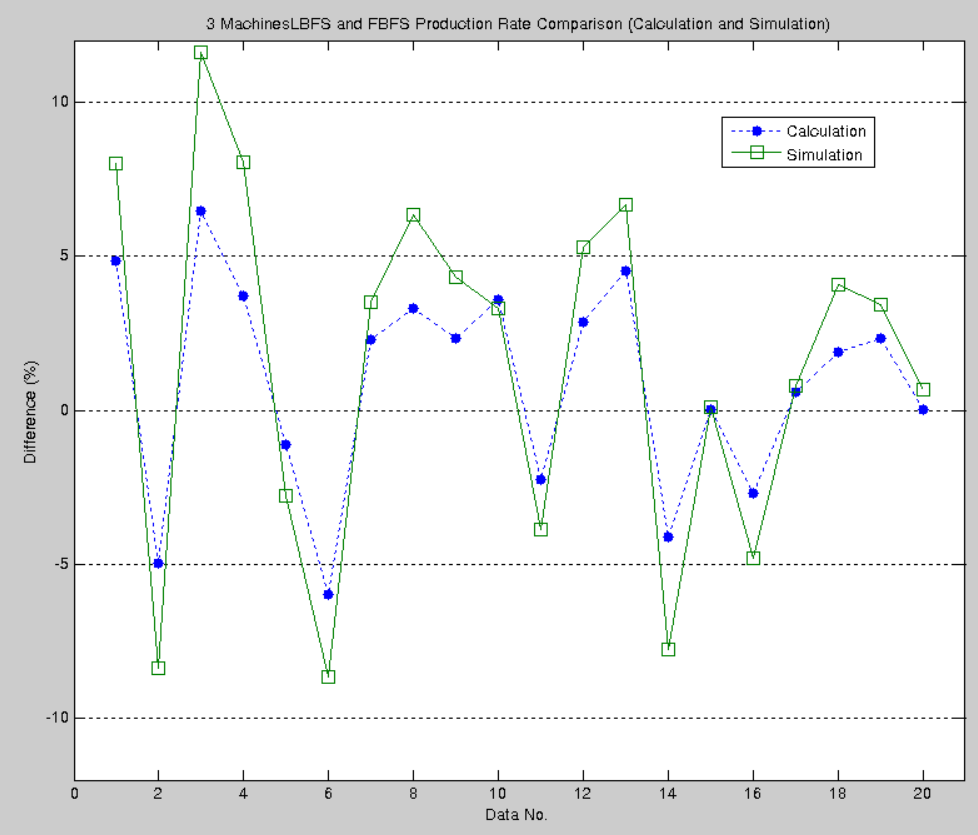

Figure 5.6 (b) PR Comparison using FBFS and LBFS (3-machine re-entrant line)

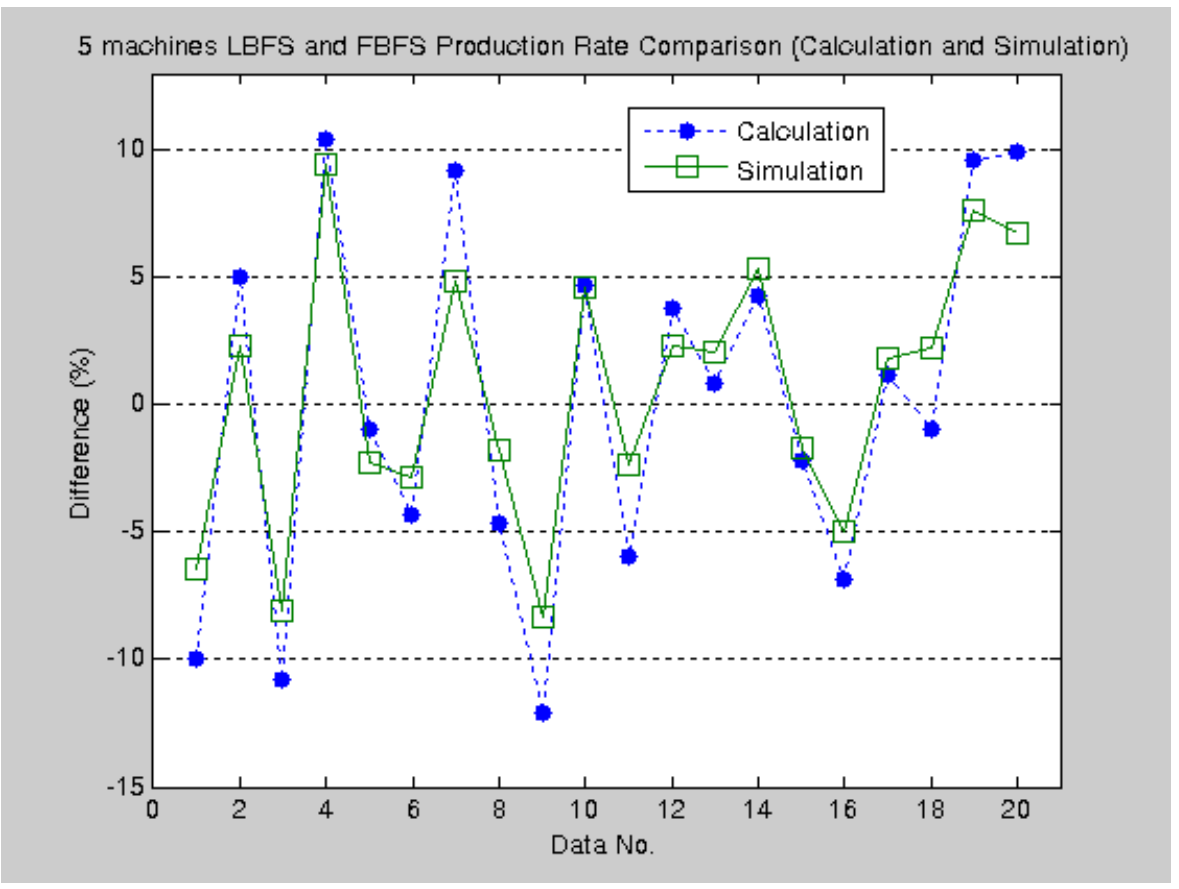

Figure 5.6 (c) PR Comparison using FBFS and LBFS (5-machine re-entrant line) 


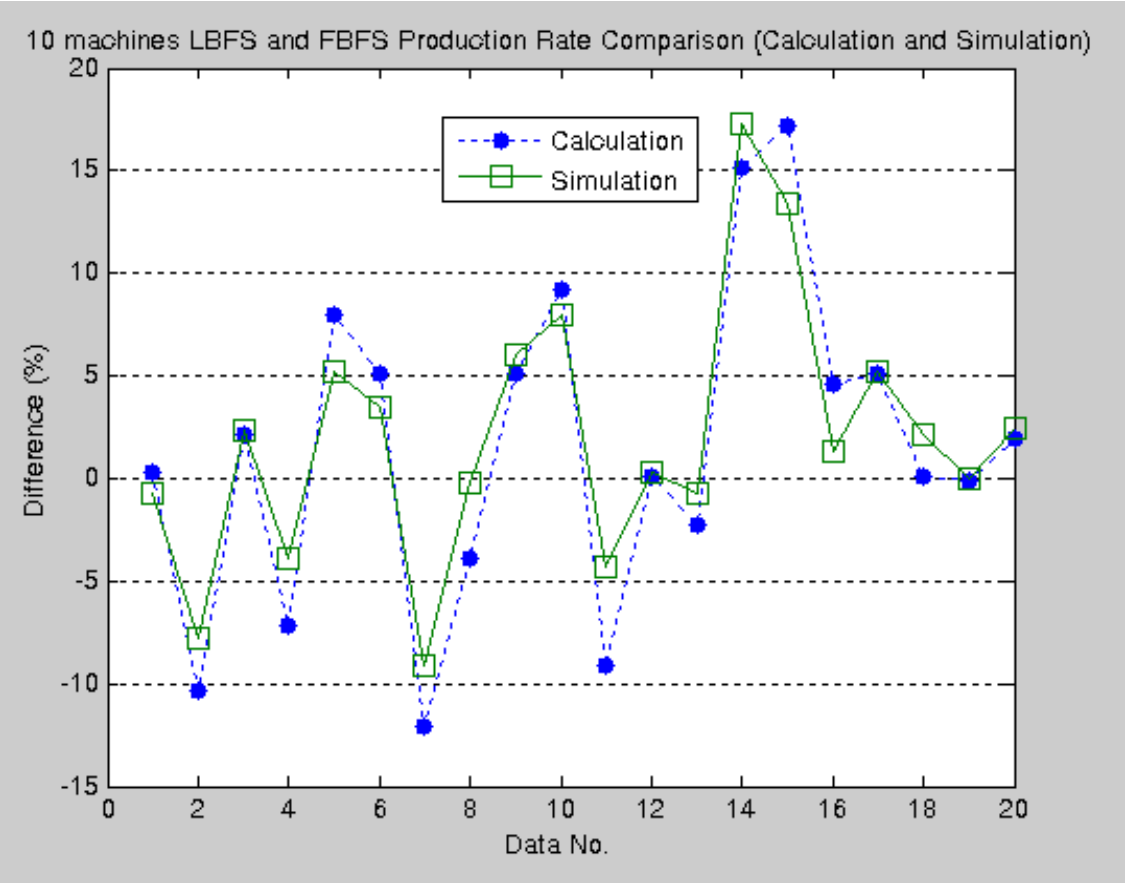

Figure 5.6 (d) PR Comparison using FBFS and LBFS (10-machine re-entrant line)

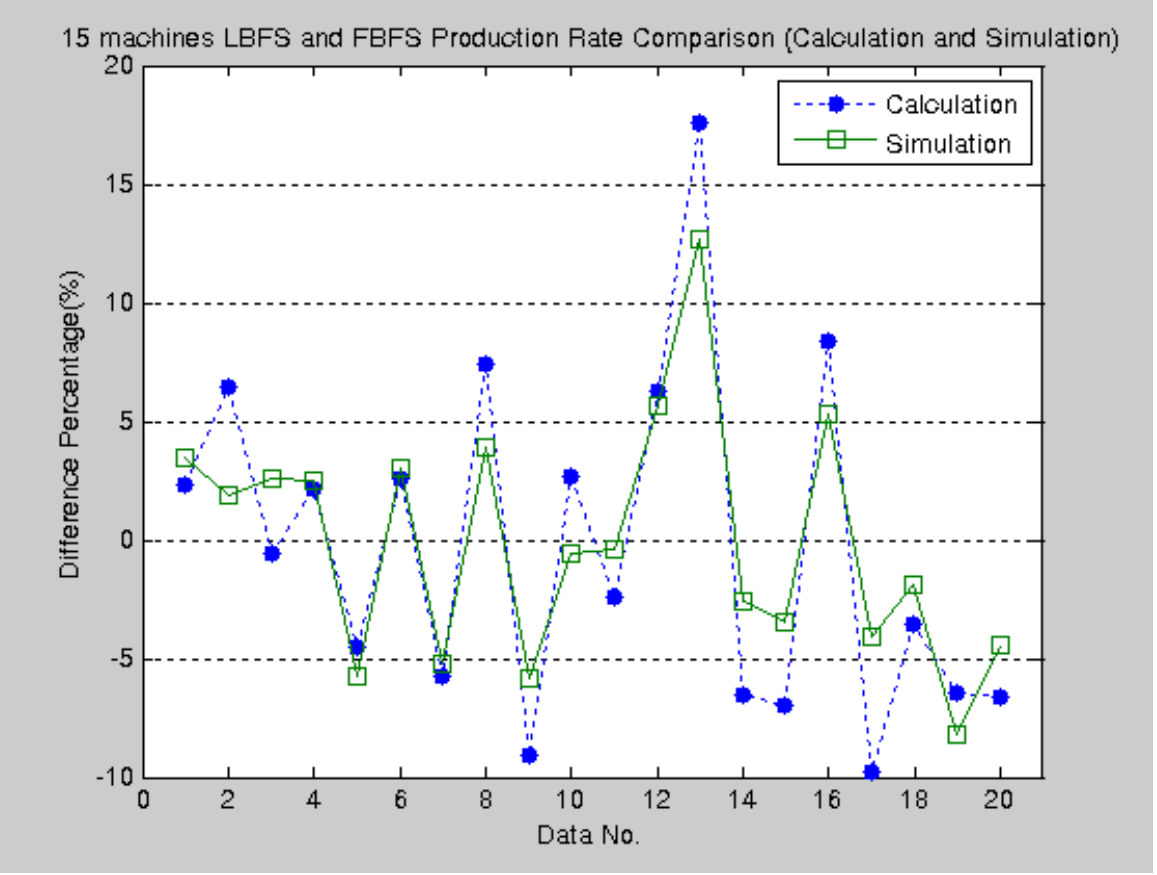

Figure 5.6 (e) $P R$ Comparison using FBFS and LBFS (15-machine re-entrant line) 


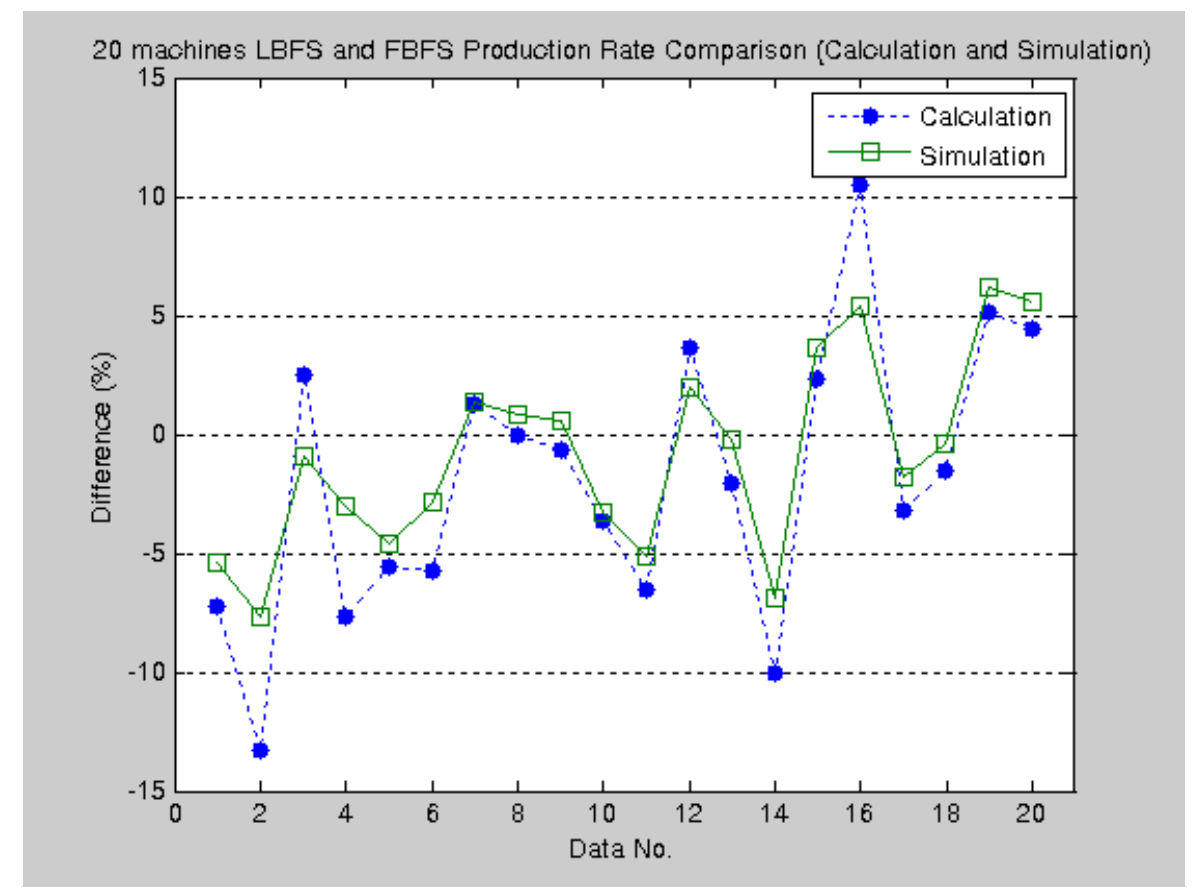

Figure 5.6 (f) PR Comparison using FBFS and LBFS (20-machine re-entrant line)

We can observe that the difference in production rate between LBFS and FBFS is relatively small, typically within $10 \%$ of the production rate of FBFS policy. Also, we can see that the results from Procedure 2 and simulation are very close. This validates the accuracy of Procedure 2 for FBFS policy. Since no significant difference in production rate is observed for LBFS and FBFS policies in the experiments, we consider lead time and WIP comparison for evaluating these two policies. Figure 5.7 shows simulation results for lead time comparison, while Figure 5.8 illustrates simulation results for WIP comparison.

Intuitively, with LBFS policy, the priority for second time job can be viewed as dragging the parts out of the production line. On the other hand, with FBFS policy, priority for first time job can be seen as pushing the parts into the 
production system. Therefore, higher WIP, longer flow time and mean queue size are as expected. The results shown in Figures 5.7 and 5.8 validate this hypothesis.

From these comparison results, we can see that FBFS policy introduces much more lead time and WIP. From Little's Law, lead time and WIP have similar increasing rates since $P R$ difference is small for FBFS and LBFS policies. Thus the comparison of WIP can be use to evaluate these two policies. Table 3 shows the ratio of $W I P_{L B F S} / W I P_{F B F S}$. As we know, reduction in lead time is of great importance for manufacturing systems. WIP is inevitably linked to cost. It is obvious that with other conditions the same, LBFS policy is better than FBFS policy in re-entrant production lines.

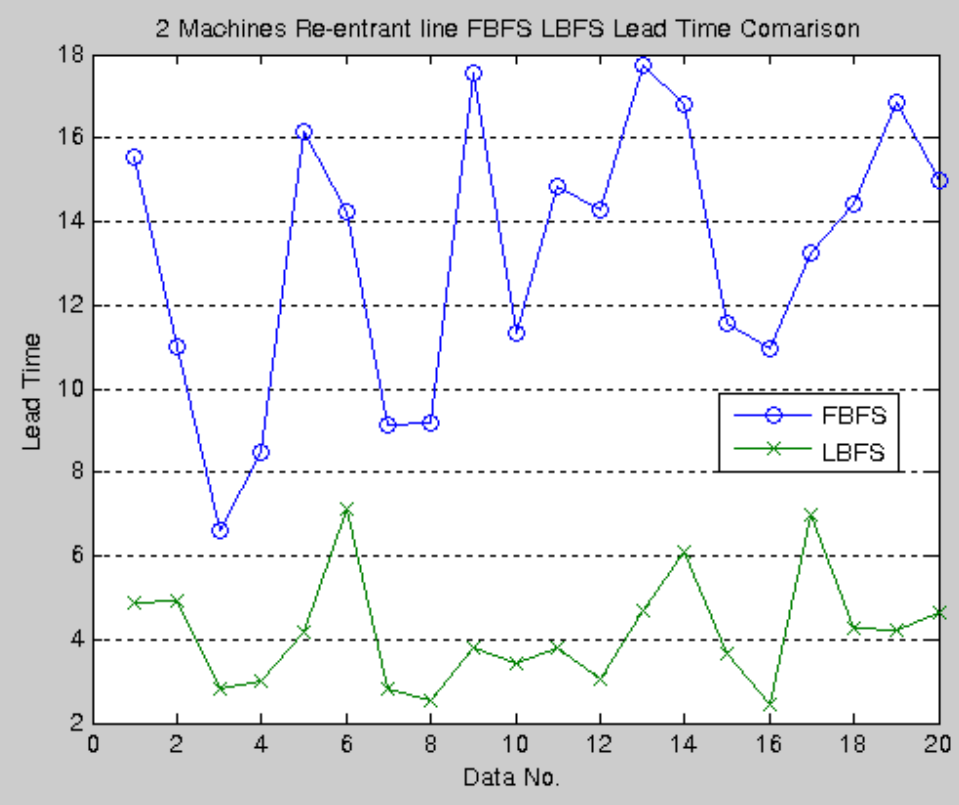

Figure 5.7 (a) Lead Time Comparison using FBFS and LBFS (2-machine reentrant line) 


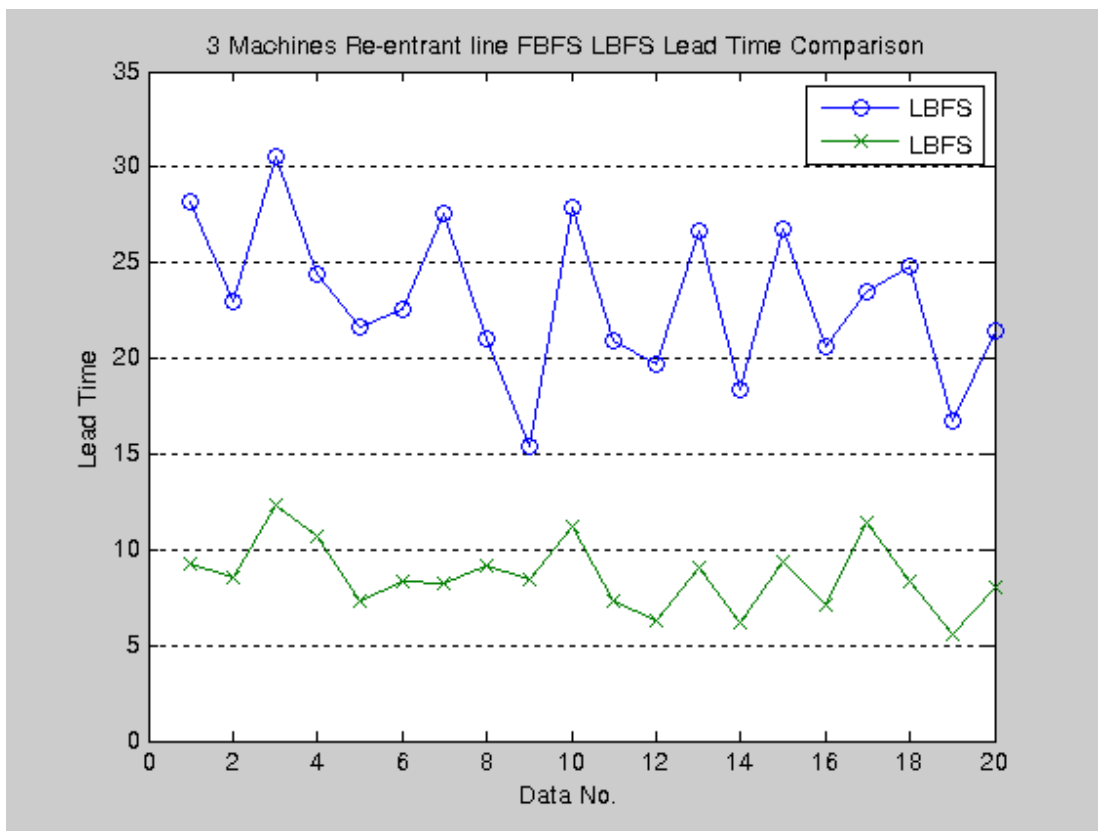

Figure 5.7 (b) Lead Time Comparison using FBFS and LBFS (5-machine reentrant line)

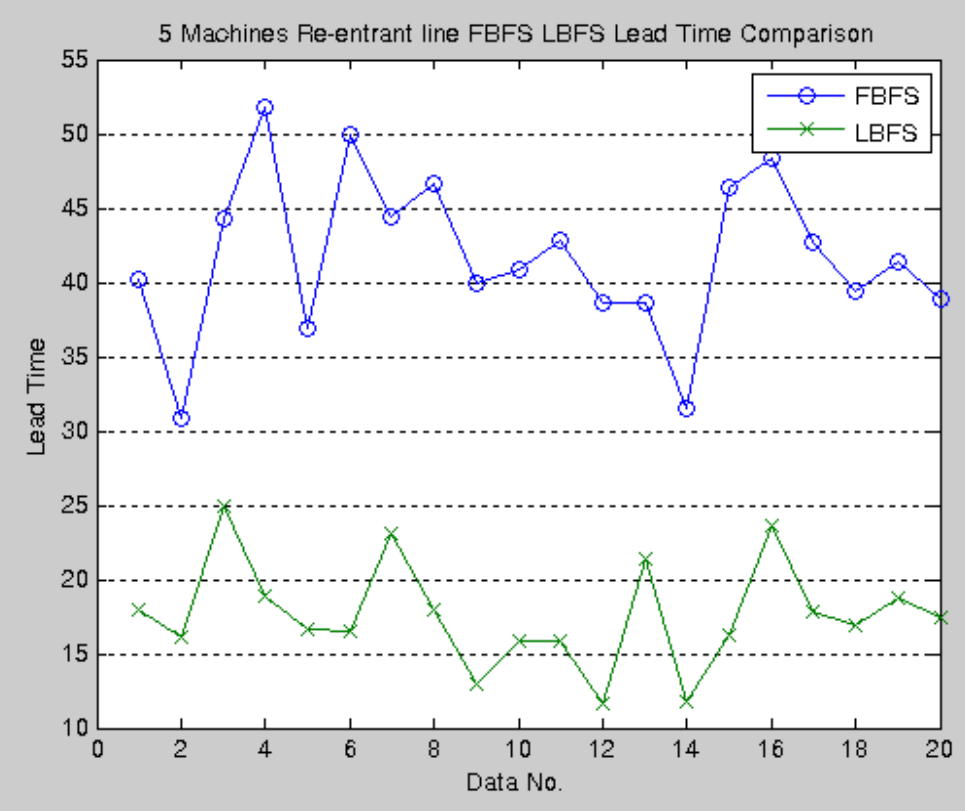

Figure 5.7 (c) Lead Time Comparison using FBFS and LBFS (5-machine reentrant line) 


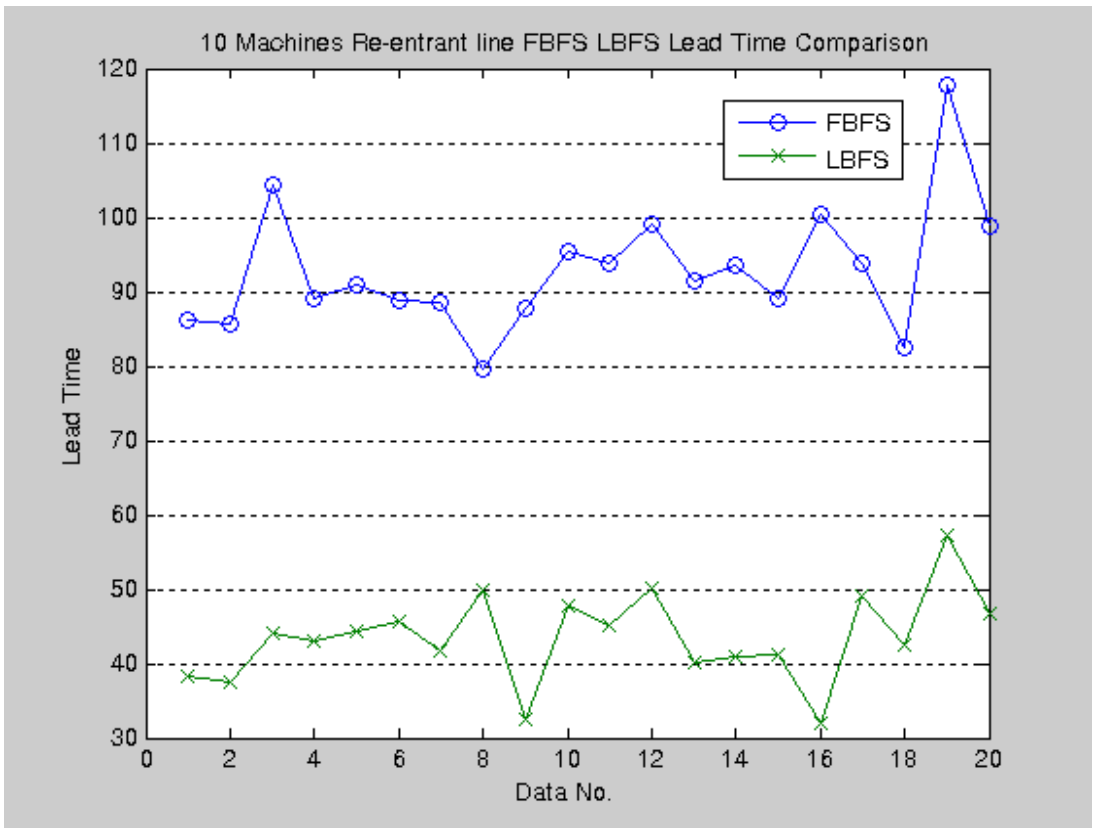

Figure 5.74 (d) Lead Time Comparison using FBFS and LBFS (10-machine reentrant line)

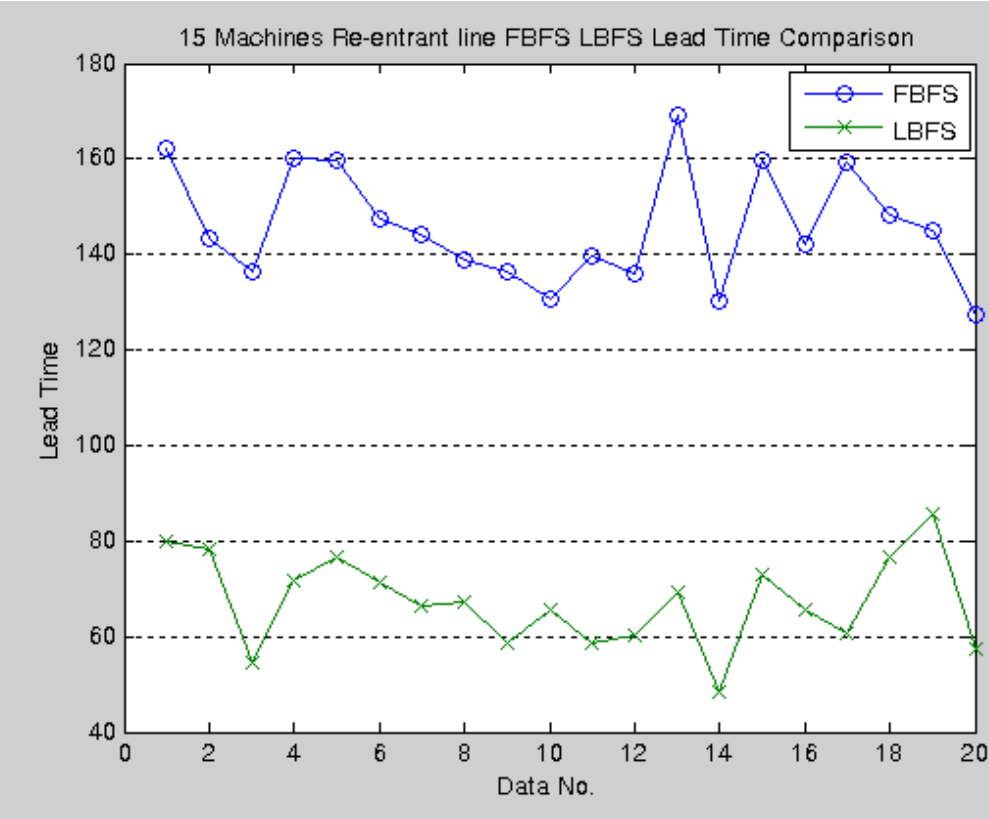

Figure 5.7 (e) Lead Time Comparison using FBFS and LBFS (15-machine reentrant line) 


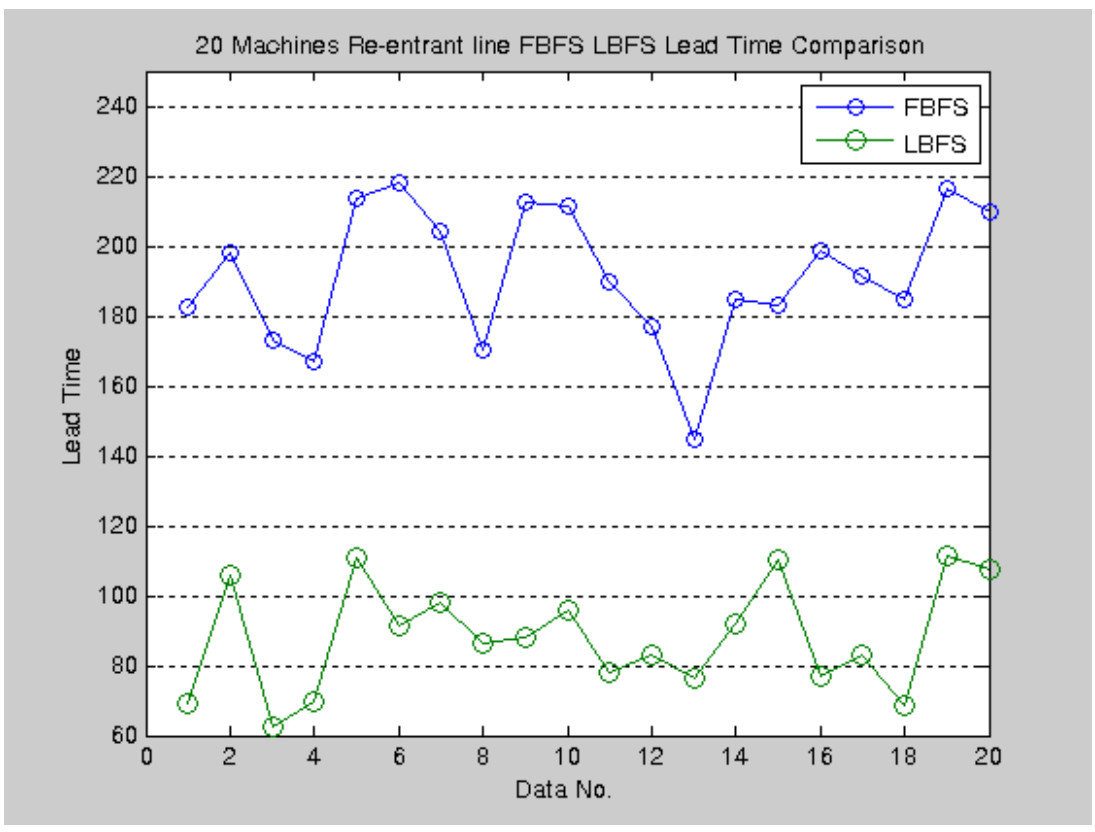

Figure 5.7 (f) Lead Time Comparison using FBFS and LBFS (20-machine reentrant line)

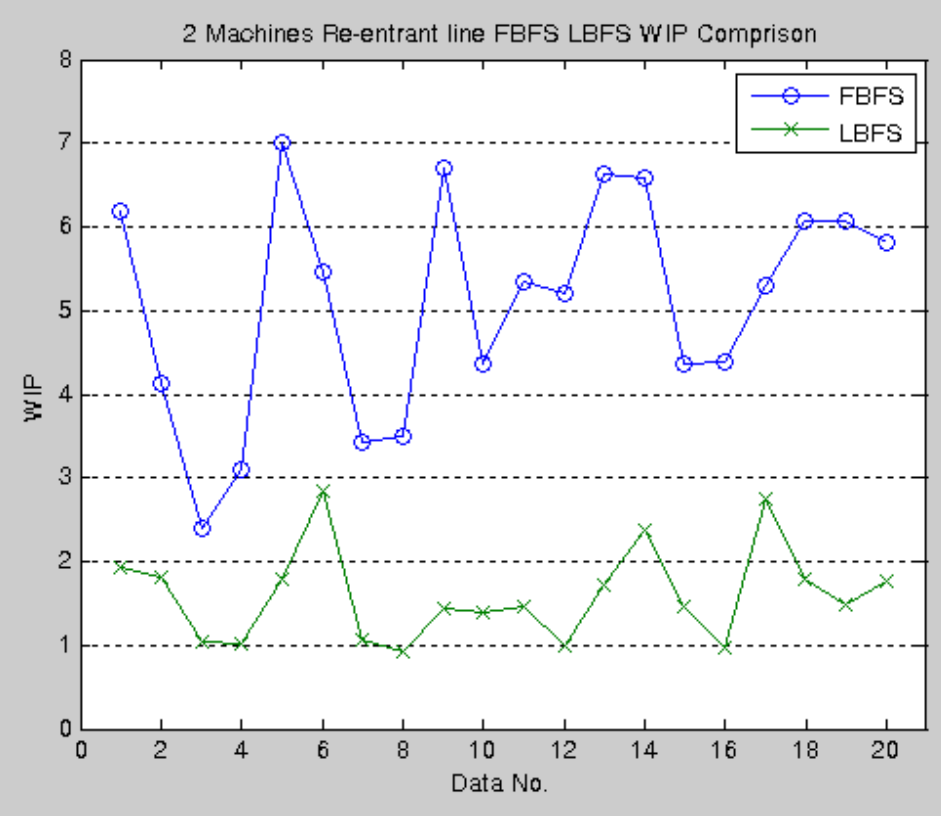

Figure 5.8 (a) WIP Comparison using FBFS and LBFS (2-machine re-entrant line) 


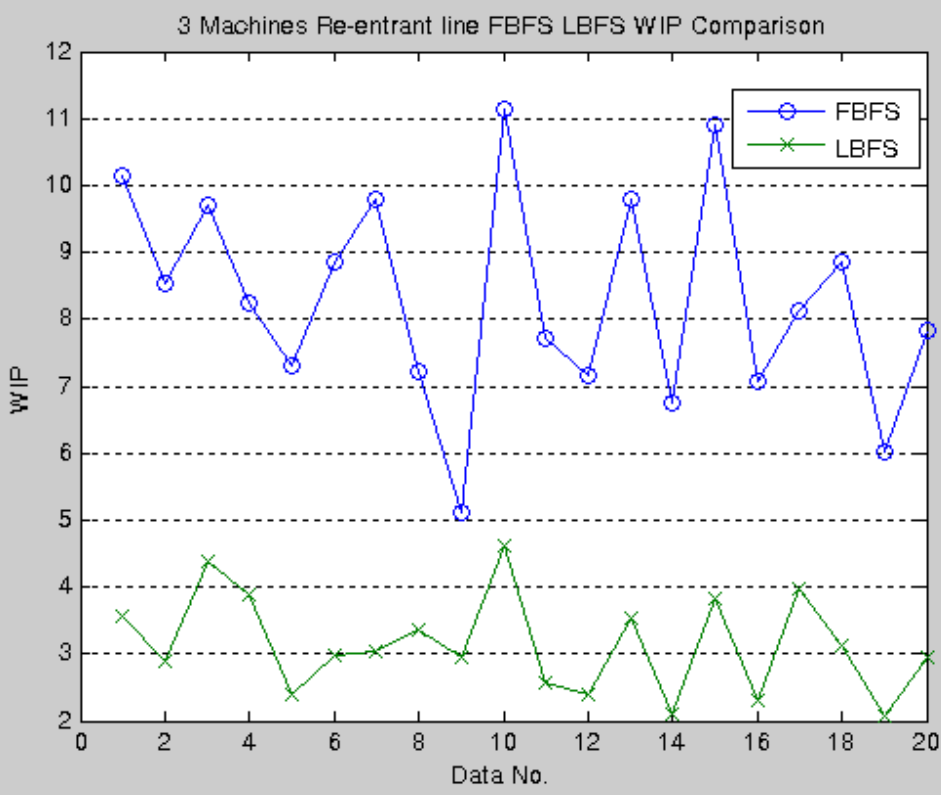

Figure 5.8 (b) WIP Comparison using FBFS and LBFS (3-machine re-entrant line)

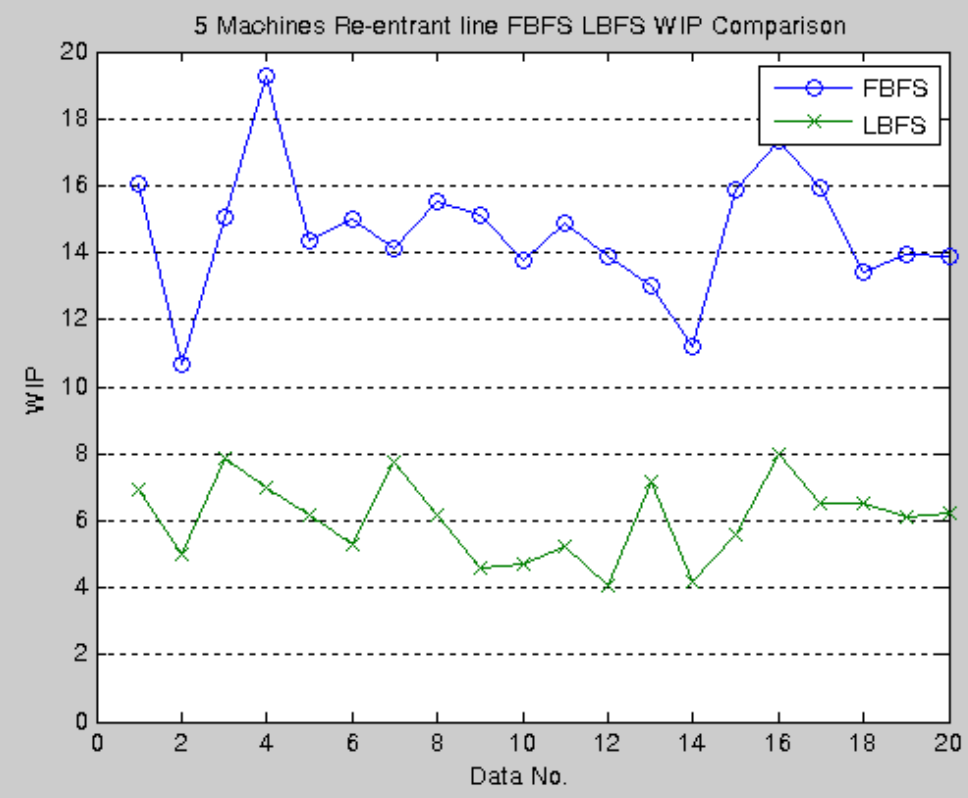

Figure 5.8 (c) WIP Comparison using FBFS and LBFS (5-machine re-entrant line) 


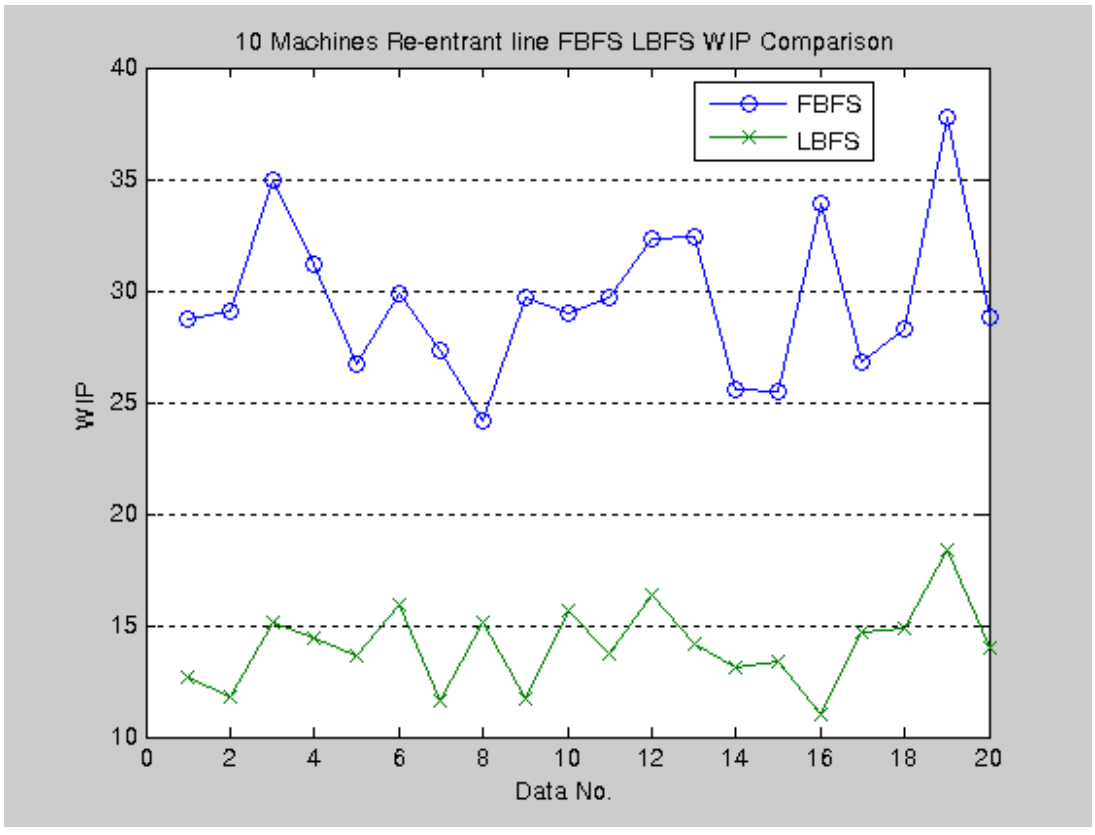

Figure 5.8 (d) WIP Comparison using FBFS and LBFS (10-machine re-entrant line)

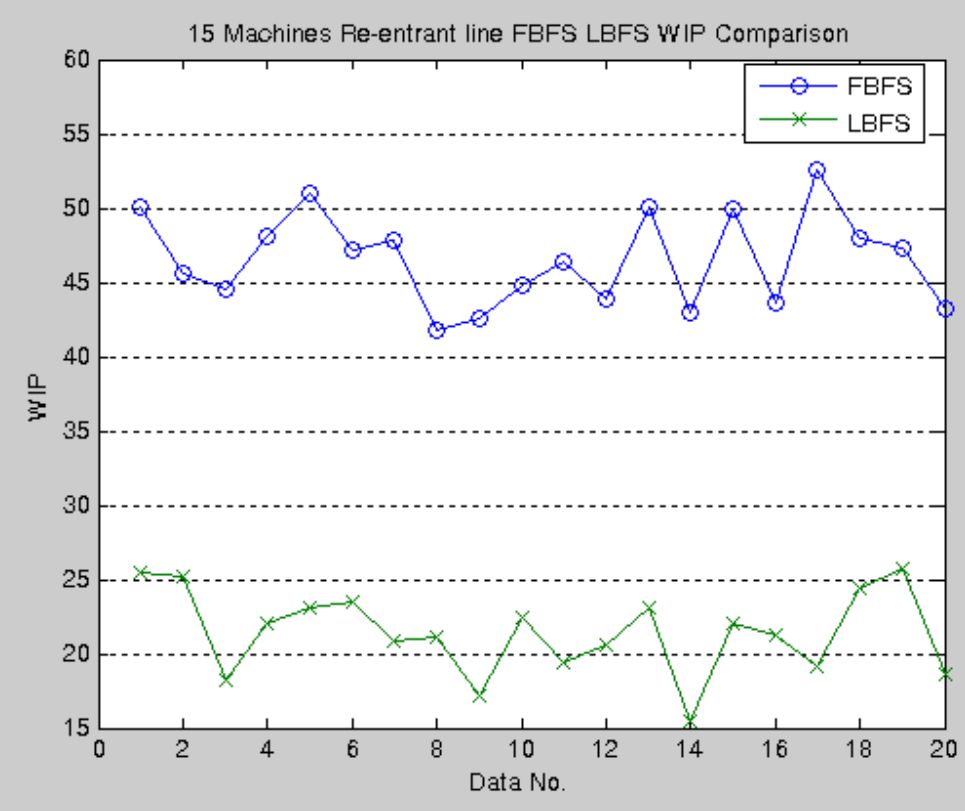

Figure 5.8 (e) WIP Comparison using FBFS and LBFS (15-machine re-entrant line) 


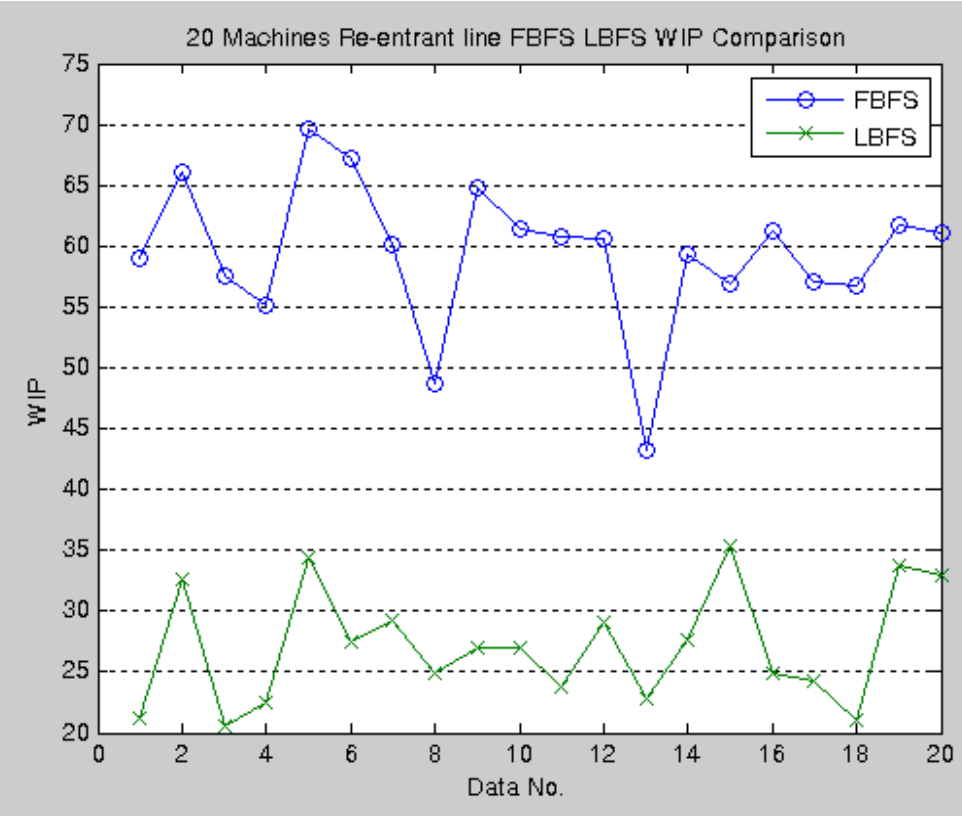

Figure 5.8 (f) WIP Comparison using FBFS and LBFS (20-machine re-entrant line)

Table 3 WIP Comparison Results

\begin{tabular}{|c|c|c|c|c|c|c|}
\hline No. of Machines & 2 & 3 & 5 & 10 & 15 & 20 \\
\hline $\operatorname{Min}\left(W_{I} P_{L B F S} / W I P_{F B F S}\right)$ & 1.91 & 1.73 & 1.82 & 1.6 & 1.8 & 1.61 \\
\hline $\operatorname{Max}\left(W I P_{L B F S} / W I P_{F B F S}\right)$ & 5.23 & 3.23 & 3.41 & 3.09 & 2.77 & 2.8 \\
\hline $\operatorname{Mean}\left(W I P_{L B F S} / W I P_{F B F S}\right)$ & 3.36 & 2.7 & 2.48 & 2.13 & 2.2 & 2.24 \\
\hline
\end{tabular}

Finally, comparing system performance using LBFS and FBFS policies, we conclude: 
- The method introduced in this work is applicable to re-entrant lines with FBFS or LBFS policies. The accuracy is similar for both policies.

- The difference in $P R$ is small.

- The differences in WIP and lead time can be significant. LBFS policy always results in smaller WIP and shorter lead time. 


\section{CHAPTER 6}

\section{CASE STUDY}

Recursive Procedures 1 and 2 have been applied to an automotive component plant to analyze the performance of an ignition processing system in the design phase. The structure of the system is illustrated in Figure 6.1. Each part has to be grinded first, cleaned by Washer 1, then polished. After that, it comes back to Washer 1 for second time cleaning. Then, it is rinsed again by Washer 2. Followed are welding operation and the final cleaning (by Washer 2 again).

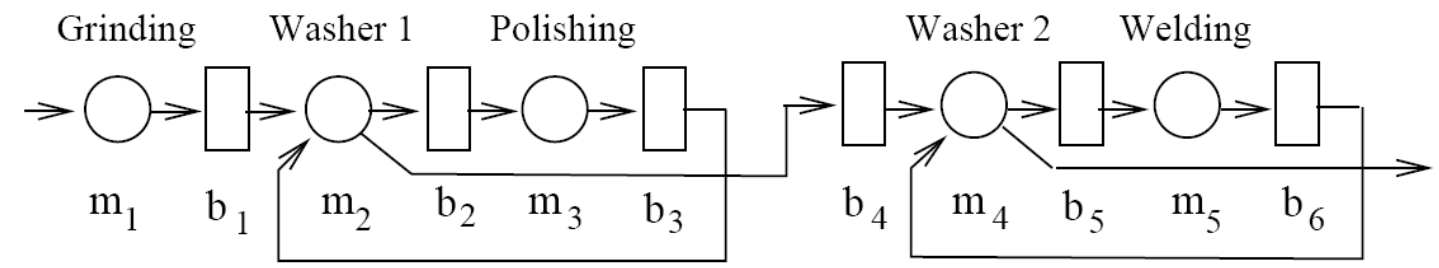

Figure 6.1 Structure of Ignition Component Processing System

In order to keep the ignition components clean, centralize washers are used to clean the components multiple times. The machine and buffer parameters are shown in Tables 4 and 5 , respectively.

By following the method developed in Chapter 4, we introduce pseudo machines $m_{2}^{\prime}$ and $m_{4}^{\prime}$ and construct a seven-machine serial line (Figure 6.2) with parameters $p_{2}^{\prime}$ and $p_{4}^{\prime}$ for machines $m_{2}^{\prime}$ and $m_{4}^{\prime}$, respectively. Using Procedures 1 and 2 , the estimated production rates are obtained as 0.4830 and 0.4876 , respectively. Compared to production rate obtained through simulation, 0.4854 , the differences are $-0.49 \%$ and $0.45 \%$, respectively, which imply that the method 
developed here provides an accurate estimate. Therefore, the model can be used for further analysis to guide the design of the system.

Table 4 Parameter of Machines

\begin{tabular}{|c|c|c|c|c|c|}
\hline & Grinding & Washer 1 & Polishing & Washer 2 & Welding \\
\hline$p_{\mathrm{i}}$ & 0.59 & 0.99 & 0.98 & 0.99 & 0.82 \\
\hline
\end{tabular}

Table 5 Parameters of Buffers

\begin{tabular}{|l|c|c|c|c|c|c|}
\hline & $b_{1}$ & $b_{2}$ & $b_{3}$ & $b_{4}$ & $b_{5}$ & $b_{6}$ \\
\hline $\mathrm{Ni}$ & 4 & 3 & 3 & 3 & 2 & 2 \\
\hline
\end{tabular}

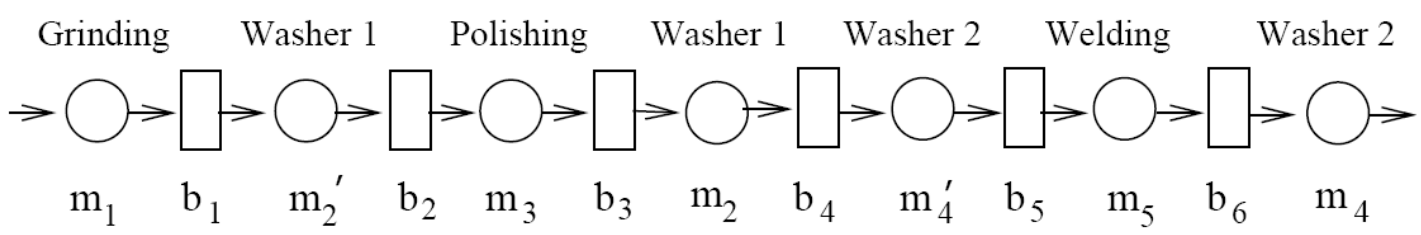

Figure 6.2 Equivalent Serial Line for Re-entrant Line in Figure 6.1 


\section{CHAPTER 7 \\ CONCLUSIONS}

Re-entrant lines are widely used in semiconductor, electronics, and many other manufacturing industries. Its design, operation, and continuous improvement deserve quick and accurate analysis of system performance. In this thesis, we present a method to approximate the system production rate of reentrant lines with Bernoulli reliability of machines. The numerical results suggest that this method can provide an acceptable precision for system production rate estimation. A case study at automotive component plant is used to illustrate the applicability of the method. In future work, the method will be extended to other machine reliability models, such as exponential, etc. The successful development of such methods will provide production engineers a quantitative tool for design and continuous improvement of re-entrant lines. 


\section{APPENDIX}

Proof of Theorem 4.1: The convergence of the procedure is proved by induction.

Step 1: For $s=0$, from initial condition (4.11) and recursive equation (4.10), we have

$$
\begin{aligned}
& p_{i}^{\prime}(1)=p_{i}-\operatorname{pr}(0), i=1, \ldots, M \\
& \operatorname{pr}(1)=\operatorname{PR}\left(p_{1}^{\prime}(1), \ldots, p_{M}^{\prime}(1), p_{1}, \ldots,=p_{M}, N_{11}, \ldots, N_{1, M_{1}}, N_{0}, N_{21}, \ldots, N_{2, M-1}\right) .
\end{aligned}
$$

Due to monotonicity of serial lines ([8]),

$$
\operatorname{pr}(1)<\operatorname{pr}(0) .
$$

Similarly

$$
\begin{aligned}
& p_{i}^{\prime}(2)=p_{i}-\operatorname{pr}(1), i=1, \ldots, M \\
& \operatorname{pr}(2)=\operatorname{PR}\left(p_{1}^{\prime}(2), \ldots, p_{M}^{\prime}(2), p_{1}, \ldots, p_{M}, N_{11}, \cdots, N_{1, M_{1}}, N_{0}, N_{21}, \ldots, N_{2, M-1}\right)
\end{aligned}
$$

Again due to monotonicity, $p_{i}^{\prime}(2)>p_{i}^{\prime}(1)$, thus

$$
\operatorname{pr}(2)>\operatorname{pr}(1) \text { and } \operatorname{pr}(2)<\operatorname{pr}(0) \text {. }
$$

Analogously, we obtain

$$
\operatorname{pr}(3)>\operatorname{pr}(1) \text { and } \operatorname{pr}(3)<\operatorname{pr}(2)
$$

which implies that

$$
\operatorname{pr}(0)>\operatorname{pr}(2)>\operatorname{pr}(3)<\operatorname{pr}(1) .
$$

Step 2: Now assume

$$
\operatorname{pr}(2 k)>\operatorname{pr}(2 k+2) \text {. }
$$

Step 3: From equation (4.10),

$$
\begin{gathered}
\operatorname{pr}(2 k+1)=\operatorname{PR}\left(p_{1}-\operatorname{pr}(2 k), \ldots, p_{M}-\operatorname{pr}(2 k), p_{1}, \ldots, p_{M},\right. \\
\left.\left.N_{11}, \ldots, N_{1, M_{1}}, N_{0}, N_{21}, \ldots, N_{2, M-1}\right)\right)
\end{gathered}
$$




$$
\begin{aligned}
\operatorname{pr}(2 k+3)= & P R\left(p_{1}-\operatorname{pr}(2 k+2), \ldots, p_{M}-\operatorname{pr}(2 k+2), p_{1}, \ldots, p_{M},\right. \\
& \left.\left.N_{11}, \ldots, N_{1, M_{1}}, N_{0}, N_{21}, \ldots, N_{2, M-1}\right)\right),
\end{aligned}
$$

and it follows that

$$
\operatorname{pr}(2 k+1)<\operatorname{pr}(2 k+3) \text { and } \operatorname{pr}(2 k)>\operatorname{pr}(2 k+1) .
$$

Similarly, from

$$
\begin{gathered}
\operatorname{pr}(2 k+4)=\operatorname{PR}\left(p_{1}-\operatorname{pr}(2 k+3), \ldots, p_{M}-\operatorname{pr}(2 k+3), p_{1}, \ldots, p_{M},\right. \\
\left.\left.N_{11}, \ldots, N_{1, M_{1}}, N_{0}, N_{21}, \ldots, N_{2, M-1}\right)\right), \\
\operatorname{pr}(2 k+3)=\operatorname{PR}\left(p_{1}-\operatorname{pr}(2 k+1), \ldots, p_{M}-\operatorname{pr}(2 k+1), p_{1}, \ldots, p_{M},\right. \\
\left.\left.N_{11}, \ldots, N_{1, M_{1}}, N_{0}, N_{21}, \ldots, N_{2, M-1}\right)\right),
\end{gathered}
$$

which implies that

$$
\operatorname{pr}(2 k+4)<\operatorname{pr}(2 k+2) \text {. }
$$

This results in

$$
\begin{aligned}
\operatorname{pr}(2 k+5)= & \operatorname{PR}\left(p_{1}-\operatorname{pr}(2 k+4), \ldots, p_{M}-\operatorname{pr}(2 k+4), p_{1}, \ldots, p_{M},\right. \\
& \left.\left.N_{11}, \ldots, N_{1, M_{1}}, N_{0}, N_{21}, \ldots, N_{2, M-1}\right)\right), \\
> & \operatorname{pr}(2 k+3) .
\end{aligned}
$$

Therefore, we obtain

$$
\operatorname{pr}(2 k+5)>\operatorname{pr}(2 k+3)>\operatorname{pr}(2 k+1) \text { and } \operatorname{pr}(2 k)>\operatorname{pr}(2 k+2)>\operatorname{pr}(2 k+4) \text {. }
$$

In addition, from (A.3), by comparing (A.1) and (A.5), we have

$$
\operatorname{pr}(2 k+1)<\operatorname{pr}(2 k+2) \text {. }
$$

Step 4: By induction, we obtain a monotonically increasing sequence $\operatorname{pr}(1)$, $\operatorname{pr}(3), \ldots, \operatorname{pr}(2 k+1), \operatorname{pr}(2 k+3), \operatorname{pr}(2 k+5), \ldots$, and a monotonically decreasing sequence $\operatorname{pr}(0), \operatorname{pr}(2), \ldots, \operatorname{pr}(2 k), \operatorname{pr}(2 k+2), \operatorname{pr}(2 k+4), \ldots$ Both sequences 
are bounded (equation (A.7)). Therefore, Procedure 1 is convergent.

Proof of Corollary 4.1: The steady state equations of (4.11) can be written as follows:

$$
\begin{gathered}
p_{i}^{\prime}=p_{i}-p r, i=1, \ldots, M, \\
p r=P R\left(p_{1}^{\prime}, \ldots, p_{M}^{\prime}, p_{1}, \ldots, p_{M}, N_{11}, \ldots, N_{1, M-1}, N_{0}, N_{21}, \ldots, N_{2, M-1}\right) .
\end{gathered}
$$

Assume there exist two solutions to (A.8), denoted as $p r$ and $\widetilde{p r}$, and $p r \neq \widetilde{p r}$. Now if

$$
p r<\widetilde{p r}
$$

we obtain $p_{i}^{\prime}>p_{i}-\widetilde{p r}:=\widetilde{p}_{i}^{\prime}$. From equation (A.8), it follows that

$$
p r>\widetilde{p r}
$$

which contradicts (A.9).

Analogously, if $p r>\widetilde{p r}$, then $p_{i}^{\prime}<\widetilde{p}_{i}^{\prime}$, again we arrive at a contradiction.

Therefore, the only possibility is $p r=\widetilde{p r}$, which implies that there is a unique solution.

Proof of Theorem 5.1: Same as Corollary 4.1, the steady state equations of (4.11) can be written as follows:

$$
\begin{gathered}
p_{i}^{\prime}=p_{i}-p r, i=1, \ldots, M, \\
p r=P R\left(p_{1}^{\prime}, \ldots, p_{M}^{\prime}, p_{1}, \ldots, p_{M}, N_{11}, \ldots, N_{1, M-1}, N_{0}, N_{21}, \ldots, N_{2, M-1}\right) .
\end{gathered}
$$

According to (4.9), when $N_{i} \rightarrow \infty$, 


$$
\begin{aligned}
p r & =\underset{N_{i} \rightarrow \infty, \forall i}{P R}\left(p_{1}^{\prime}, \ldots, p_{M}^{\prime}, p_{1}, \ldots, p_{M}, N_{11}, \ldots, N_{1, M-1}, N_{0}, N_{21}, \ldots, N_{2, M-1}\right) \\
& =\min \left(p_{1}^{\prime}, \ldots, p_{M}^{\prime}, p_{1}, \ldots, p_{M}\right) \\
& =\min \left(p_{1}-p r, \ldots, p_{M}-p r\right) \\
& =\min \left(p_{i}\right)-p r, i=1, \ldots, M,
\end{aligned}
$$

therefore

$$
p r=\lim _{N_{i} \rightarrow \infty, \forall i} P R=\min \left(p_{i}\right) / 2
$$

Proof of Theorem 5.2: We use Procedure 1 to prove this corollary. The corresponding argument with respect to Procedure 2 follows immediately.

First we show the monotonicity with respect to machine reliability. Consider two re-entrant lines. Line 1 has machines $p_{1}, \ldots, p_{i-1}, p_{i}, p_{i+1}, \ldots, p_{M}$, and Line 2 has $p_{1}, \ldots, p_{i-1}, \tilde{p}_{i}, p_{i+1}, \ldots, p_{M}$. Both lines have same buffer capacities. Denote the production rates of these two lines as $p r$ and $\widetilde{p r}$, respectively.

Assume that $p_{i}<\tilde{p}_{i}, i \in\{1, \ldots, M\}$, we need to show that this leads to $p r<\widetilde{p r}$. To accomplish this, first we assume $p r \geq \widetilde{p r}$, i.e.,

$$
\begin{aligned}
& p r=P R\left(p_{1}-p r, \ldots, p_{i}-p r, \ldots, p_{M}-p r, p_{1}, \ldots, p_{i}, \ldots, p_{M},\right. \\
& \left.\quad N_{11}, \ldots, N_{1, M-1}, N_{0}, N_{21}, \ldots, N_{2, M-1}\right) \\
& \geq P R\left(p_{1}-p r, \ldots, \widetilde{p}_{i}-\widetilde{p r}, \ldots, p_{M}-p r, p_{1}, \ldots, p_{i}, \ldots, p_{M},\right. \\
& \left.\quad N_{11}, \ldots, N_{1, M-1}, N_{0}, N_{21}, \ldots, N_{2, M-1}\right) \\
& =\widetilde{p r} .
\end{aligned}
$$

However, due to monotonicity of serial lines, $p_{i}<\tilde{p}_{i}$, and from assumption 
(A.10), we have

$$
\begin{aligned}
p r< & P R\left(p_{1}-p r, \ldots, \widetilde{p}_{i}-p r, \ldots, p_{M}-p r, p_{1}, \ldots, \tilde{p}_{i}, \ldots, p_{M},\right. \\
& \left.N_{11}, \ldots, N_{1, M-1}, N_{0}, N_{21}, \ldots, N_{2, M-1}\right) \\
\leq & P R\left(p_{1}-\widetilde{p r}, \ldots, \widetilde{p}_{i}-\widetilde{p r}, \ldots, p_{M}-\widetilde{p r}, p_{1}, \ldots, \tilde{p}_{i}, \ldots, p_{M},\right. \\
& \left.N_{11}, \ldots, N_{1, M-1}, N_{0}, N_{21}, \ldots, N_{2, M-1}\right) \\
=\widetilde{p r} &
\end{aligned}
$$

which is a contradiction to (A.10). Therefore, we must have $p r<\widetilde{p r}$, i.e., the system production rate is monotonically increasing with respect to $p_{i}$. Next, we show that production rate is monotonically increasing with respect to buffer capacity. Again consider two production lines, both have identical machines, and Line 1 has buffer capacities $N_{11}, \ldots, N_{i}, \ldots, N_{2, M-1}$, and Line 2 has $N_{11}, \ldots, \widetilde{N}_{i}, \ldots, N_{2, M-1}$. Assume that $N_{i}<\widetilde{N}_{i}, i \in\{0,11, \ldots,(1, M-1), 21, \ldots,(2, M-1)\}$, we need to show that $p r<\widetilde{p r}$, where, as before, $p r$ and $\widetilde{p r}$ are production rates of Lines 1 and 2, respectively. Again we assume $p r \geq \widetilde{p r}$, i.e.,

$$
\begin{aligned}
p r & =P R\left(p_{1}-p r, \ldots, p_{M}-p r, p_{1}, \ldots, p_{M}, N_{11}, \ldots, N_{i}, \ldots, N_{2, M-1}\right) \\
\geq & P R\left(p_{1}-\widetilde{p r}, \ldots, p_{M}-\widetilde{p r}, p_{1}, \ldots, p_{M}, N_{11}, \ldots, \widetilde{N}_{i}, \ldots, N_{2, M-1}\right) \\
& =\widetilde{p r}
\end{aligned}
$$

Due to monotonicity of serial lines, $N_{i}<\widetilde{N}_{i}$, and from assumption (A.11), 


$$
\begin{aligned}
p r & <\operatorname{PR}\left(p_{1}-p r, \ldots, p_{M}-p r, p_{1}, \ldots, p_{M}, N_{11}, \ldots, \widetilde{N}_{i}, \ldots, N_{2, M-1}\right) \\
& <\operatorname{PR}\left(p_{1}-\widetilde{p r}, \ldots, p_{M}-\widetilde{p r}, p_{1}, \ldots, p_{M}, N_{11}, \ldots, \widetilde{N}_{i}, \ldots, N_{2, M-1}\right) \\
& =\widetilde{p r} .
\end{aligned}
$$

Tt is a contradiction to assumption (A.11). Therefore, the only possibility is $p r<\widetilde{p r}$, i.e., the system production rate is monotonically increasing with respect to buffer capacity $N_{i}$.

\section{Proof of Theorem 5.3}

This theorem is proved by contradiction. First we consider Procedure 1. In the original line,

$$
p r=P R\left(p_{1}-p r, \ldots, p_{M}-p r, p_{1}, \ldots, p_{M}, N_{11}, \ldots, N_{1 M}, N_{0}, N_{21}, \ldots, N_{2, M-1}\right) .
$$

In the reversed line,

$$
\widetilde{p r}=P R\left(p_{M}, \ldots, p_{1}, p_{M}-\widetilde{p r}, \ldots, p_{1}-\widetilde{p r}, N_{2, M-1}, \ldots, N_{21}, N_{0}, N_{1 M}, \ldots, N_{11}\right),
$$

where $\widetilde{p r}$ denotes the production rate of the reversed line.

Using the reversibility property (4.8) in serial lines ([8]), we have

$$
\widetilde{p r}=P R\left(p_{1}-\widetilde{p r}, \ldots, p_{M}-\widetilde{p r}, p_{1}, \ldots, p_{M}, N_{11}, \ldots, N_{1 M}, N_{0}, N_{21}, \ldots, N_{2, M-1}\right) .
$$

If $p r>\widetilde{p r}$, due to monotonicity, $p_{1}-p r>p_{1}-\widetilde{p r}$, thus $p r<\widetilde{p r}$, which is a contradiction. Similarly, if $p r<\widetilde{p r}$, contradiction also occurs. Therefore, the only possibility is $p r=\widetilde{p r}$. 
For Procedure 2, similar proof can be obtained. 


\section{REFERENCES}

[1] Y. Dallery and S.B. Gershwin, "Manufacturing Flow Line Systems: A Review of Models and Analytical Results," Queuing Systems, vol. 12, pp. 3-94, 1992.

[2] H.T. Papadopoulos and C. Heavey, "Queueing Theory in Manufacturing Systems Analysis and Design: A Classification of Models for Production and Transfer Lines," European Journal of Operational Research, vol. 92, pp. 1-27, 1996.

[3] J. Li, D.E. Blumenfeld, N. Huang and J.M. Alden, "Throughput Analysis in Production Systems: Recent Advances and Future Topics," submitted to IEEE Transactions on System, Man, Cybernetics - Part C, 2007.

[4] N. Viswanadham and Y. Narahari, Performance Modeling of Automated Manufacturing System, Prentice Hall, 1992.

[5] J.A. Buzacott and J.G. Shantikumar, Stochastic Models of Manufacturing Systems, Prentice Hall, 1993.

[6] H.T. Papadopoulos, C. Heavey and J. Browne, Queueing Theory in Manufacturing Systems Analysis and Design, Chapman \& Hall, 1993.

[7] S.B. Gershwin, Manufacturing Systems Engineering, PTR Prentice Hall, 1994.

[8] J. Li and S.M. Meerkov, Production Systems Engneering, WingSpan Press, 2007.

[9] L.M. Wein, "Schedling Semiconductor Wafter Fabrication," IEEE Transactions on Semicondutor Manufacturing, vol. 1, pp. 115-130, 1988.

[10] P.R. Kumar, "Schedling Semiconductor Manufacturing Plants," IEEE Control Systems Magazine, vol. 14, pp. 30-40, 1994. 
[11] M.C. Zhou and M.D. Jeng, "Modeling, Analysis, Simulation, Scheduling, and Control of Semiconductor Manufacturing Systems: A Petri Net Approach," IEEE Transactions on Semiconductor Manufacturing, vol. 11, pp. 333-357, 1998.

[12] S. Kumar and P.R. Kumar, "Queueing Network Models in the Design and Analysis of Semiconductor Wafer Fabs," IEEE Transactions on Robotics and Automation, vol. 17, pp. 548-561, 2001.

[13] W.J. Hopp, M.L. Spearman, S. Chayet, K.L. Donohue and E.S. Gel, "Using an Optimized Queueing Network Model to Support Wafer Fab Design," IIE Transactions, vol. 34, pp. 119-130, 2002.

[14] P.R. Kumar, "Re-entrant Lines," Queueing Systems, vol. 13, pp. 87-110, 1993.

[15] P.R. Kumar, "Scheduling Manufacturing Systems of Re-entrant Lines," in Stochastic Modeling and Analysis of Manufacturing Systems, Ed. D.D. Yao, pp. 325-360, Springer-Verlag, New York, 1994.

[16] Y. Narahari and L.M. Khan, "Performance Analysis of Scheduling Policies in Re-entrant Manufacturing Systems," Computers \& Operations Research, vol. 23, pp. 37-51, 1996.

[17] S. Kim, Y. Park and C. Jun, "Performance Evaluation of Re-entrant Manufacturing System with Production Loss Using Mean Value Analysis," Computers \& Operations Research, vol. 33, pp. 1308-1325, 2006.

[18] M.D. Jeng, X.L. Xie and W.Y. Hung, "Markovian Timed Petri Nets for Performance Analysis of Semiconductor Manufacturing Systems," IEEE Transactions on Systems, Man and Cybernetics - Part B, vol. 30, pp. 757-771, 
2000.

[19] J.Y. Choi and S.A. Reveliotis, "A Generalized Stochastic Petri Net Model for Performance Analysis and Control of Capacitated Re-entrant Lines," IEEE Transactions on Robotics and Automation, vol. 20, pp. 474-480, 2003.

[20] J. Li, "Performance Analysis of Production Systems with Rework Loops," IIE Transactions, vol. 36, pp. 755-765, 2004.

[21] C. Wang and J. Li, "Approximate Analysis of Re-entrant Lines with Bernoulli Reliability of Machines," Report PSL-07-01, Dept. of Electrical and Computer Engineering, University of Kentucky, Lexington, KY.

[22] J. G. Shanthikumar, S. Ding, Zhang, Queueing Theory for Semiconductor Manufacturing Systems: A Survey and Open Problems IEEE Transaction on Automation Science and Engineering vol.4 Issue. 4, pp. 513-522, 2007.

[23] S.H. Lu, and P.R. Kumar,"Distributed scheduling based on due dates and buffer priorities", IEEE Transactions on Automatic Control, 36, 1406-1416, 1991.

[24] M. Bramson, and J.G. Dai, "Heavy traffic limits for some queueing networks", Annals of Applied Probability, vol 11, 1, 49-90,1999.

[25] H. Chen, "Fluid approximations and stability of multiclass queueing networks: Work-conserving disciplines", Annals of Applied Probability, 5, 637-655, 1995. 
[26] J.G. Dai, "On positive Harris recurrence of multiclass queueing networks: a unified approach via fluid limit models", Annals of Applied Probability, 5, 49-77, 1995.

[27] C. Bispo,, Tayur, S., "Managing simple re-entrant flow lines: I. A theoretical framework, " GSIA Working paper, January 1997.

[28] L. M. Wein, "Scheduling semiconductor wafer fabrication," IEEE Transactions on Semiconductor Manufacturing, vol. 1, pp. 115-130, 1988. 


\section{VITA}

Name: Chong Wang

Date of Birth: April, 22, 1980

Place of Birth: Shenyang, China

\section{Education:}

BS Material Science Engineering, Tianjin University, 2003

BS Electrical Engineering, $\quad$ Tianjin University, 2003

MS Precision Measurement Technology and Instruments

Tianjin University, 2006 